\title{
WATER DROPLETS AS FILLERS: \\ THE EFFECT OF A DISPERSED AQUEOUS PHASE ON THE RHEOLOGY AND MICROSTRUCTURE OF FAT CRYSTAL-STABILIZED WATER-IN-OIL EMULSIONS
}

By

\author{
Ruby Rose Rafanan
}

B. Sc. Ryerson University (2009)

M. Sc. Ryerson University (2013)

A dissertation presented to Ryerson University in

partial fulfillment of the requirements for the

degree of Doctor of Philosophy in

the program of Molecular Science

Toronto, ON

C Copyright Ruby Rose Rafanan (2019) 


\section{AUTHOR'S DECLARATION FOR ELECTRONIC SUBMISSION OF A DISSERTATION}

I hereby declare that I am the sole author of this dissertation. This is a true copy of the dissertation, including any required final revisions, as accepted by my examiners.

I authorize Ryerson University to lend this dissertation to other institutions or individuals for the purpose of scholarly research

I further authorize Ryerson University to reproduce this dissertation by photocopying or by other means, in total or in part, at the request of other institutions or individuals for the purpose of scholarly research.

I understand that my dissertation may be made electronically available to the public. 


\section{Abstract}

Composite systems are continuous matrices that contain embedded particles, called fillers, that can be used to manipulate the mechanical strength and viscoelastic properties of the resulting material. The use of fillers in composite systems is common practice and largely depends on particle size and shape, surface functional groups and aggregation behaviour. Emulsions are complex food dispersions and, while they have structural similarities in common with composites, they are not widely considered in this light. In this thesis, fat crystal network-stabilized emulsions prepared using various emulsifiers were studied to determine whether varying the structure of the water droplet surface could modulate the viscoelastic behaviour of the resulting composite. The oil phase consisted of a high melting triglyceride in canola oil with an emulsifier that promoted a specific droplet surface, namely polyglycerol polyricinoleate (PGPR) which did not promote interfacial crystallization, or one of two monoglycerides [glycerol monooleate (GMO), or glycerol monostearate (GMS)], which both enhanced interfacial crystallization of fats.

Interfacial fat crystallization imparted structural rigidity by surrounding the dispersed aqueous droplets within a solid shell that interacted with adjacent network crystal aggregates and other droplets. The work here shows that the presence of interfacial fat causes the droplet to promote an increase in viscosity and in $\mathrm{G}^{\prime}$, thus reinforcing the crystal network. These effects were enhanced by decreasing the droplet size and increasing the volume fraction of the dispersed aqueous phase. As well, the crystalline shell that provided imparted active filler qualities to the droplets also protected the droplet from shear degradation, protecting encapsulated contents from being released. 
PGPR, a branched, polymeric emulsifier, minimized the presence of interfacial fat crystallization such that interactions of the dispersed droplets interacted only weakly with nearby droplets and fat crystals. As a result, the dispersed phase did not have a pronounced effect on emulsion reinforcement or viscosity and displayed predominantly viscous behaviour irrespective of volume fraction. These aqueous droplets were also less effective as encapsulation vehicles as they were more prone to shear-mediated release of encapsulated material.

These results show that the droplet interface is determinant in fat crystal network rheology and shear-stability by establishing the dispersed phase droplets of such emulsions as either active or inactive fillers. Mechanically strong droplets (i.e., those with interfacial fat crystal shells) which interacted strongly with their surroundings more effectively reinforced/added rigidity to the emulsion - behaviour typical of active fillers. Conversely, the presence of weakly interacting droplets (i.e., liquid interface) did not impart changes to emulsion rheology. As with solid filler particles, droplet characteristics, such as size and volume fraction can then be used to predictably modulate viscoelastic behaviour and encapsulation efficiency. Filler "activity" can also be used as a tool to control encapsulation or release of compounds during shear. This thesis experimentally demonstrates that embedded droplets with interfaces tailored by the use of surfactants can be considered a functional and tunable component of fat crystal network-stabilized emulsions. 


\section{Acknowledgements}

First and foremost, thank you to Dérick Rousseau, my thesis supervisor, for his understanding, guidance and patience throughout my graduate career. Without your support, I would not have been able to achieve all that I did. Thank you for helping me find my way.

To my supervisory committee members, Drs. Supratim Ghosh, Scott Tsai, and Stephen Wylie, thank you for your feedback and valuable perspectives throughout my PhD.

I would like to thank my lab group members, past and present, homegrown and international, for their thoughtful input and constructive critiques. I am very fortunate to have worked with such a great group. To my senior members, Nicole Green, Ryan West, and Tu Tran, the food adventures, the constant snacking and one-way coffee were vital to the completion of my research. To my undergraduate volunteers-turned-thesis students, Andre Castillo and Melinda Dass, I am so grateful for your enthusiasm, your persistence and humour. The research couldn't have been done without you.

Special acknowledgements go to my mother, Delia, and my partner, Mathew Petrus. Both of you were so understanding throughout this very long process. I am very thankful to have the support of you two incredible people. 


\section{Table of Contents}

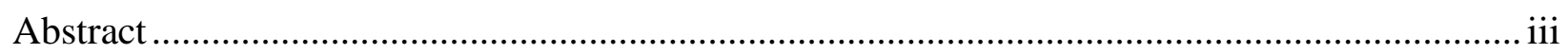

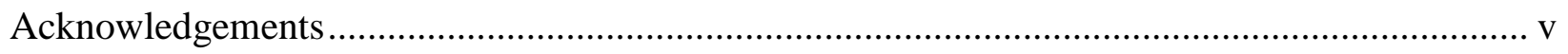

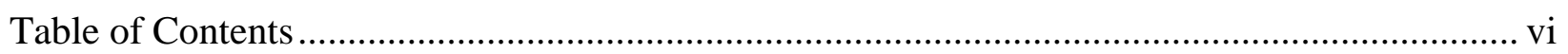

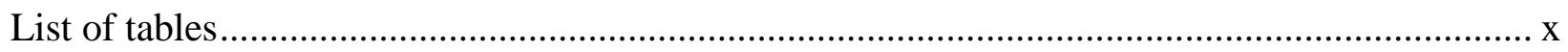

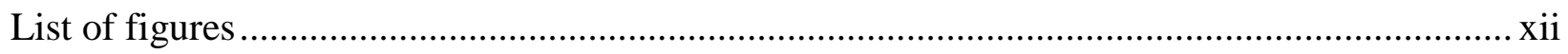

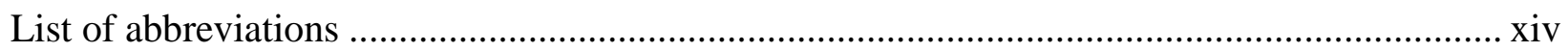

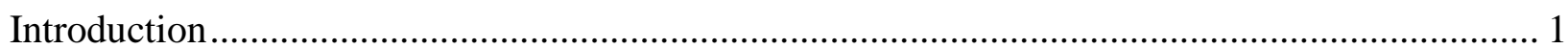

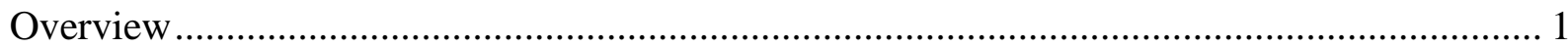

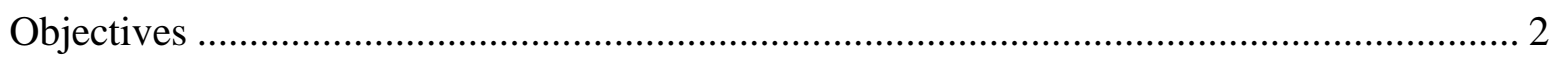

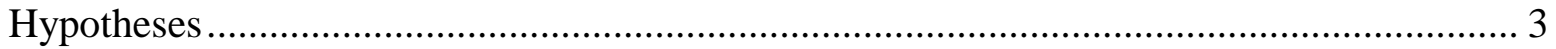

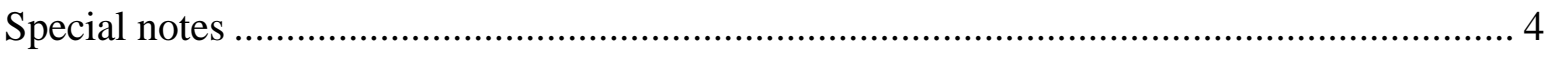

Chapter 1 - Water droplets as fillers in water-in-oil food emulsions....................................... 5

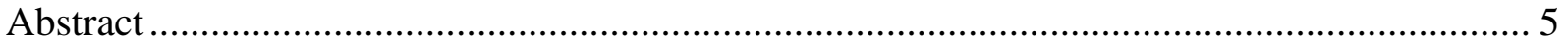

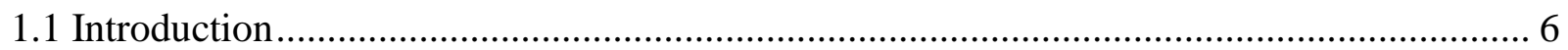

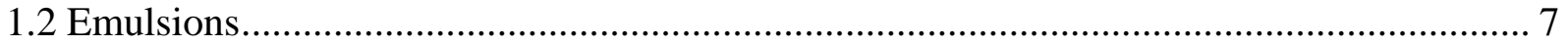

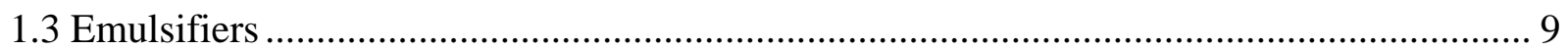

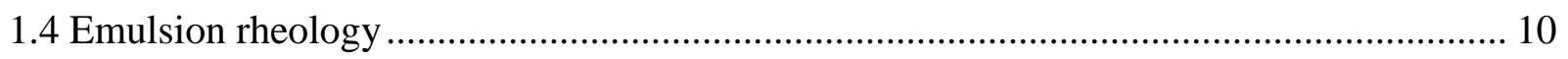

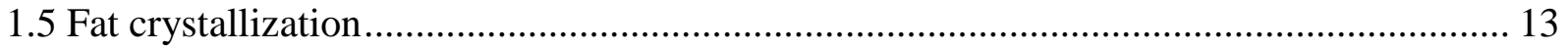

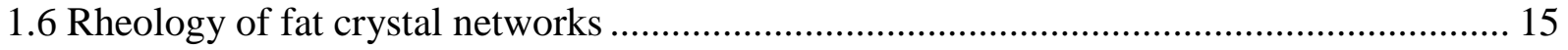

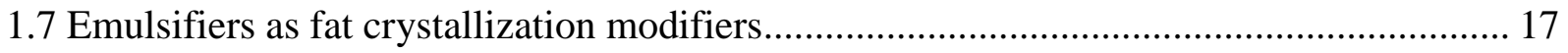

1.8 Role of fat crystals in stabilization of W/O emulsions .............................................. 18

1.8.1 Pickering particles for emulsion stabilization ................................................... 19 
1.8.2 Core-shell particle emulsion stabilization.............................................................. 19

1.9 Rheology of fat crystal-stabilized W/O emulsions .......................................................... 20

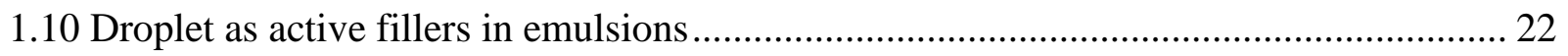

10.1 Droplet as active fillers in W/O emulsions within a fat crystal network ...................... 26

1.11 Applications of fat crystal-stabilized W/O emulsions ..................................................... 32

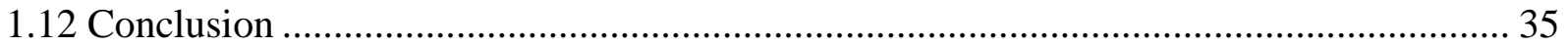

Chapter 2 - Dispersed droplets as active fillers in fat-crystal network-stabilized water-in-oil

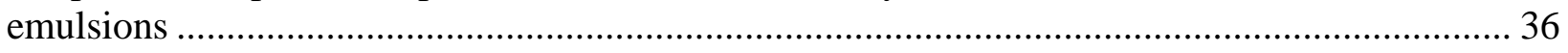

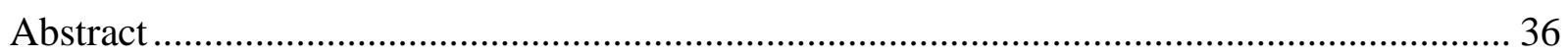

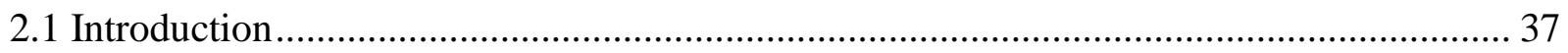

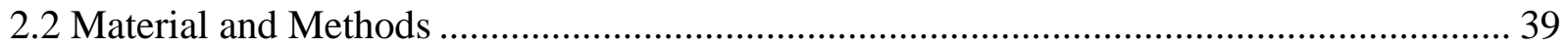

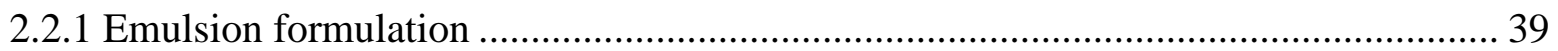

2.2.2 Solid fat content and fat crystal network characterization .......................................... 41

2.2.3 Microscopy and droplet size determination ............................................................... 42

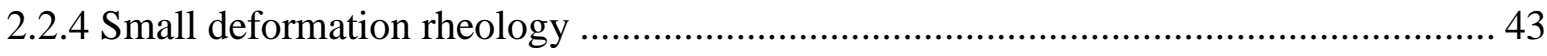

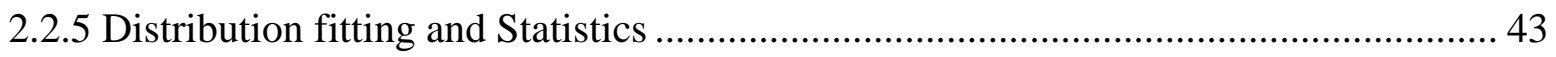

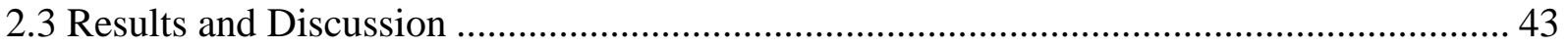

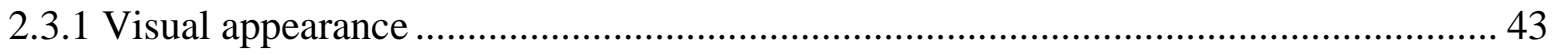

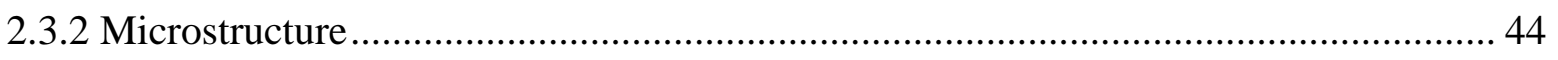

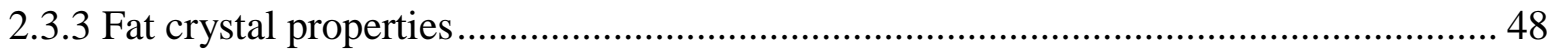

2.3.4 Emulsion and bulk fat rheology .............................................................................. 50

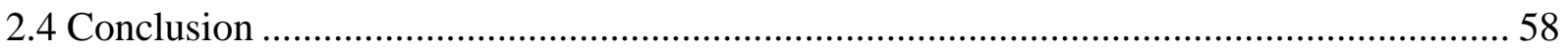

Chapter 3 - Dispersed droplets as tunable fillers in water-in-oil emulsions stabilized with fat

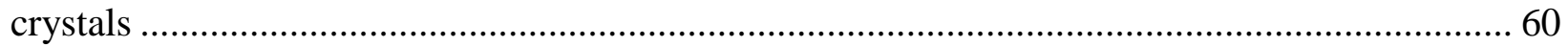

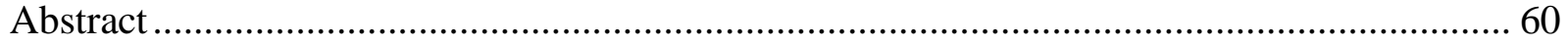

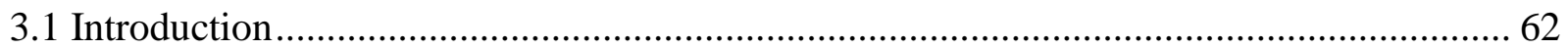




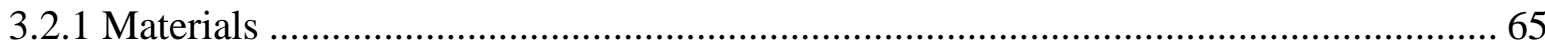

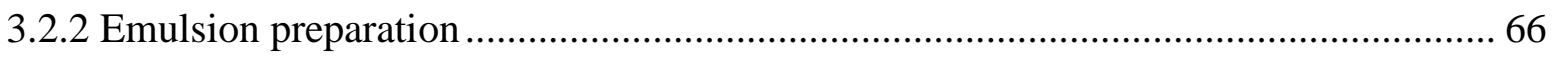

3.2.3 Solid fat content and droplet diameter with pulsed NMR .......................................... 69

3.2.4 Microscopy ……………………………………................................................ 70

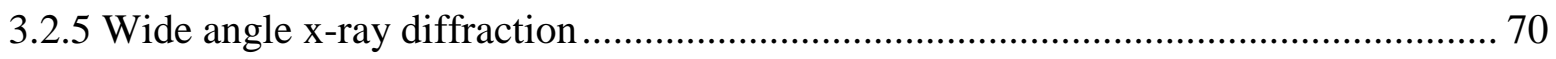

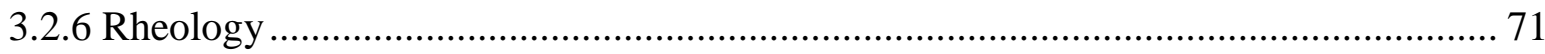

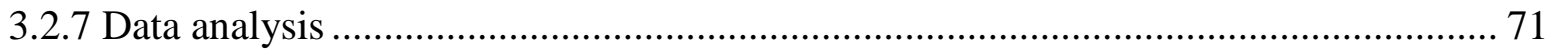

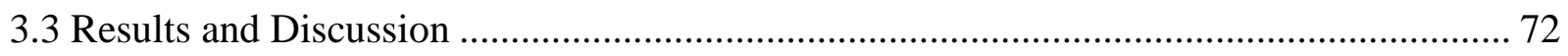

3.3.1 Visual appearance and dispersed phase droplet size............................................... 72

3.3.2 Fat crystal network properties.............................................................................. 74

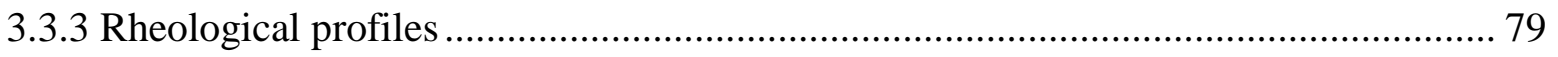

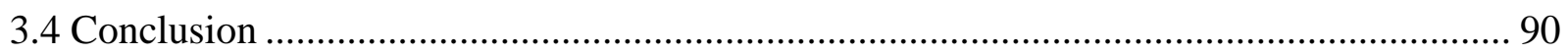

Chapter 4 - Effect of shear and interfacial fat crystallization on release of water-soluble dye from

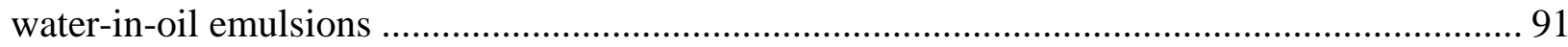

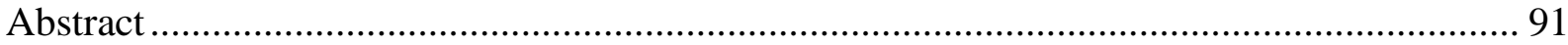

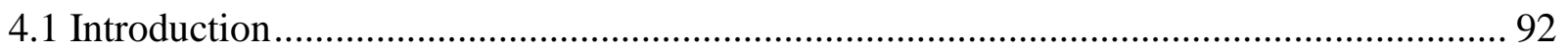

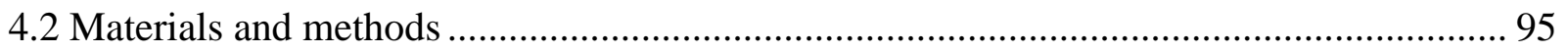

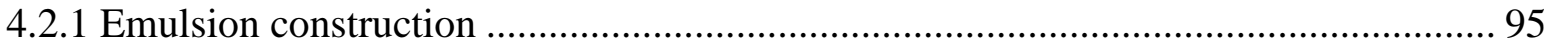

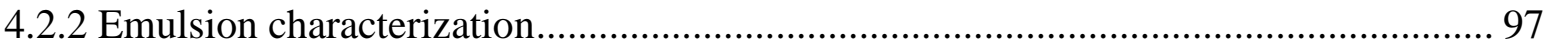

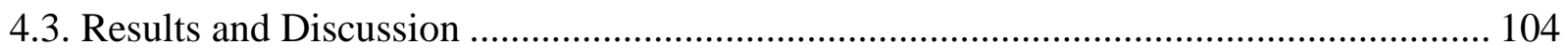

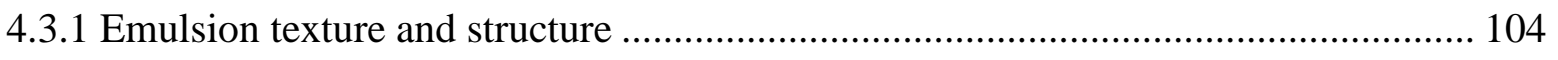

4.3.2 Confocal image analysis ................................................................................... 108

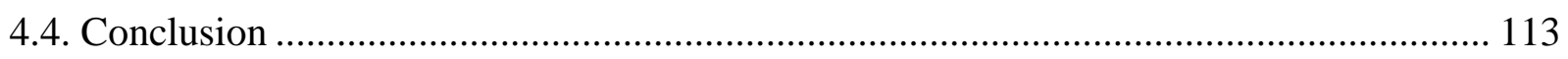

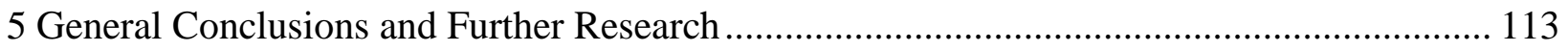

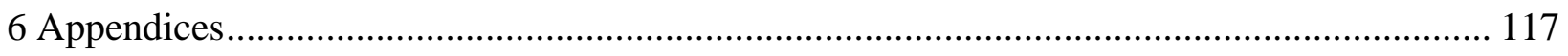

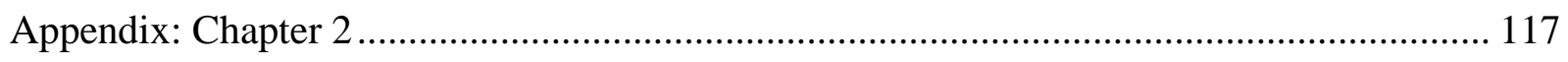




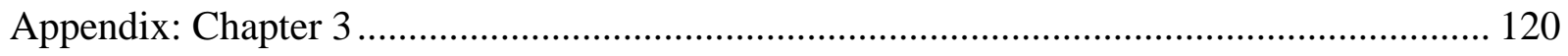

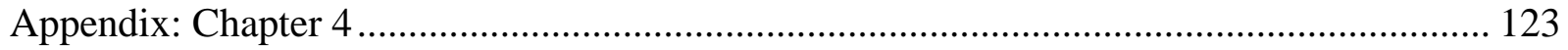

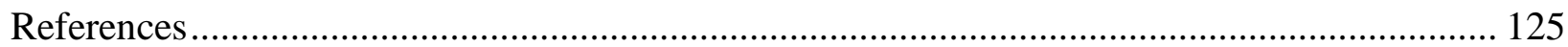




\section{List of tables}

Table 2.1 - Emulsion composition. Total emulsion mass was 200g.

Table 2.2: Solid fat content of HSO in the W/O emulsions as a function of time, water content and emulsifier type

Table 2.3: Melting enthalpy $(\mathrm{J} / \mathrm{g})$ of HSO in the W/O emulsions as a function of time, water content and emulsifier type

Table 2.4: Power law model fit of frequency sweep $G^{*}$ values for fat crystal network-stabilized emulsions containing 0 - $20 \mathrm{wt} \%$ water over time, where $\mathrm{G}^{*}=\mathrm{a} \omega^{\mathrm{b}}$

Table 3.1- Emulsion and blank compositions (wt.\%)

Table 3.2 - Homogenization conditions for MAG-stabilized emulsions to attain different droplet size distributions

Table 3.3 - Homogenization conditions for PGPR-stabilized emulsions to attain different droplet size distributions

Table 3. 4 - Dominant TAG crystal polymorphs identified in emulsions made with HSO and either GMS, PGPR or GMO over time

Table 4.1: Emulsion compositions on wt $\%$ basis

Table 4.2: Physical characteristics of fat crystal network-stabilized emulsions (Droplet diameter, SFC, apparent viscosity at $1.6 \mathrm{~s}^{-1}$, melting point and enthalpy)

Appendix table 2.1 - Droplet size distributions for all fat crystal-stabilized emulsions as determined by bright field microscopy image analysis 
Appendix table 2.2 - Peak melting temperatures $\left({ }^{\circ} \mathrm{C}\right)$ of $\mathrm{HSO}$ in the W/O emulsions as a function of time, water content and emulsifier type

Appendix table 3.1 - Power law fitting of emulsion viscosity - $a$ coefficient Appendix table 3.2 - Power law fitting of emulsion viscosity $-b$ coefficient 


\section{List of figures}

Figure 1.1: Interactions between a filled gel matrix and filler particles

Figure 1.2A - Major factors dictating rheology in emulsions

Figure 1.2B - Major factors dictating rheology in fat crystal networks

Figure 1.2C - Major factors dictating rheology in composite systems to show the intersectionality of structural interactions

Figure 2.1 - Appearance of freshly-prepared $20 \mathrm{wt} \% \mathrm{~W} / \mathrm{O}$ emulsions prepared with (A) PGPR and (B) GMO

Figure 2.2 - Micrographs of freshly-prepared $20 \mathrm{wt} \% \mathrm{~W} / \mathrm{O}$ emulsions containing either GMO or PGPR

Figure 2.3 - The amplitude-dependent storage modulus $\left(\mathrm{G}^{\prime}\right)$ of GMO and PGPR-based emulsions at days 0 and 7

Figure 2.4 - Frequency sweeps from 0.01 to $10 \mathrm{~Hz}$ of GMO and PGPR-based emulsions at days 0 and 7

Figure 3.1 - Visual appearance of the different fat crystal-stabilized emulsions

Figure 3.2 - Brightfield (upper panel) and polarized light (lower panel) microscopy of fat crystal-stabilized emulsions prepared with PGPR, GMS, or GMO

Figure 3.3 - Evolution in $\mathrm{G}^{\prime}(\mathrm{kPa})$ of emulsions made with PGPR, GMS, or GMO

Figure 3.4 - Evolution in Reinforcement index (R) of emulsions made with PGPR, GMS, or GMO over time 
Figure 3.5 - Apparent viscosity at low shear (1 rpm) and high shear (100 rpm)

Figure 3.6 - Schematic summary of the role droplet size, and particle interaction strength on fat crystal network rheology

Figure 4.1: Schematic of Rhodamine B release set-up and microscopy preparation

Figure 4.2 Confocal image of typical gel cross-sections after dye was applied to the surface

Figure 4.3 Comparison of macroscopic texture W/O emulsions stabilized by PGPR. GMO and GMS

Figure 4.4 Droplet size distributions of fat crystal-stabilized emulsions

Figure 4.5 Microscopic morphology of emulsions

Figure 4.6 Microscopic morphology of emulsions after shear treatment

Figure 4.7: Rhodamine path length given in $\mu \mathrm{m}$ from the gel surface

Figure 4.8 Release mechanism of dye from water droplets in a fat crystal network

Appendix figure 2.1 - Frequency sweep data from $0.01-10 \mathrm{~Hz}$ at $0.02 \%$ strain for fat crystal network stabilized emulsions

Appendix figure 3.1 - XRD scattering of different HSO polymorphs

Appendix figure 4.1 - Average dye intensity within the gel as a function of distance from the gel surface which begins at $\mathrm{x}=0$

Appendix figure 4.2 - Melting curves of the PGPR, GMS and GMO stabilized emulsions determined by DSC at a heating rate of $5^{\circ} \mathrm{C} / \mathrm{min}$ 


\section{List of abbreviations}

DSC - differential scanning calorimetry

DSD - droplet size distribution

GMO - glycerol monooleate

GMS - glycerol monostearate

EE - Encapsulation efficiency

HSO - hydrogenated soy oil

LVR - linear viscoelastic region

MAG - monoacylglycerol

NMR - nuclear magnetic resonance

$\mathrm{O} / \mathrm{W}$ - oil-in-water

PGPR - polyglycerol polyricinoleate

$\mathrm{RCF}$ - relative centrifugal force

SFC - solid fat content

TAG - triacylglycerol

W/O - water-in-oil 
XRD - X-ray diffraction 


\section{Introduction}

\section{Overview}

Fat crystal networks are effective for improving the stability of water-in-oil emulsions by providing a steric barrier that prevents droplet coalescence and adds structural strength to the material. Much research has been done to account for the contributions of the fat crystal network on bulk rheology, however comparatively less work has been done to account for the contributions of the dispersed phase.

Structurally, emulsions resemble composite systems, as they consist of a continuous network with embedded particles that, together, will dictate mechanical rigidity, viscosity and other rheological properties. The embedded materials, referred to as fillers, can be active or inactive, depending on whether their presence increases structural rigidity (active filler) or reduces integrity/imparts no effect (inactive filler). The "activity" of the filler can be modulated by controlling different aspects of the particles, most notably surface functional groups, volume fraction, aggregative tendency, surface roughness and particle size. Judicious manipulation of these specific filler characteristics can control the rheology of the overall material. The use of filler particles and study of their properties is common in the materials science field, but is not applied to food systems in spite structural similarity and useful application.

It is the purpose of the work here to describe emulsion droplets as liquid fillers. Just as solid particles are adjustable in terms of size and surface structure, emulsion droplets can be modified through processing conditions and use of surfactants to create droplets with structure necessary to elicit a desired rheological response. Oil droplets in oil-in-water emulsion gels have been 
described previously in terms of their filler potential, but not water droplets within oil-continuous matrices. The work presented here describes how water droplets in fat crystal network-stabilized emulsions can behave as functional filler particles in a composite by controlling droplet characteristics to alter viscoelastic behaviour of the network.

Chapter 1 examines fat crystal network-stabilized emulsions as composite systems and factors influencing filler activity. Chapter 2 examines how the structure of the droplet interface and volume fraction of the dispersed phase affects rheology. Chapter 3 describes how droplet size affects rheology and how these effects are dependent on the droplet interface. Chapter 4 deals with how the interface influences the release of encapsulated material when under shear conditions. The final chapter summarizes the major findings of this research and identifies further research to expand on this body of work.

\section{Objectives}

The overarching objective of this thesis was to better describe how dispersed emulsion droplets within a fat crystal network behave as rheology-modifying filler particles by modulating specific characteristic.

The following objectives were as follows:

i. To determine whether active or inactive filler effects could be promoted by the presence of droplets with interfacial crystals or with a liquid interface, respectively (Chapter 2).

ii. To determine how volume fraction and droplet size distribution affect the viscoelastic behaviour of active and inactive filler droplets within a fat crystal network (Chapters 2 and 3). 
iii. To determine the effect of shear on the release behaviour of a water-soluble dye encapsulated within liquid surfactant- and interfacial crystal-stabilized droplets from a fat crystal network-stabilized emulsion (Chapter 4).

\section{Hypotheses}

Surface characteristics have a strong impact on the viscoelastic behaviour of classical composite (solid particulate embedded within a matrix) systems. In water-in-oil fat crystal network-stabilized emulsions, where water droplets are embedded within a matrix, the droplet interface may promote viscoelastic changes on the network as a solid particle would. It is hypothesized that:

i. For fat crystal network-stabilized emulsions whose droplets exhibit interfacial crystallization, these droplets will impose an active filler effect resulting in more rigid network emulsions resulting from stronger filler-matrix associations compared to a liquid interface. A liquid stabilized interface has comparatively weaker associations and do not contribute to network rigidity (Chapter 2).

ii. As active filler volume fraction increases, the number of attractive interactions between filler droplets and their surroundings also increases, resulting in an increase in network rigidity and viscosity. Conversely, inactive fillers form weaker interactions and will not reinforce the network substantially, irrespective of the volume fraction or surface area (Chapter 2 and 3).

iii. Interfacial crystallization provides an added barrier against shear deformation and droplet disintegration which provides superior encapsulation during shear over liquid surfactant-stabilized droplets (Chapter 4). 


\section{Special notes}

All experiments described in this manuscript were performed by me, with the exception of Chapters 1 and 2 where the partial NMR data was collected by Andre Castillo and assorted microscopy/ImageJ image analysis for droplet size distribution was performed by Melinda Dass. The articles that form the basis for Chapters 2-4 were written by me as the first author with Dérick

Rousseau. Chapters 2 and 3 have been published in Food Research International (2017) and Journal of Food Engineering (2019). Chapter 4 was accepted for publication in LWT and was published in May 2019. 


\title{
Chapter 1 - Water droplets as fillers in water-in-oil food emulsions
}

\author{
Abstract \\ There is increasing interest in water-in-oil (W/O) emulsions with enhanced functionality to \\ develop foods with a reduced saturated fat content, lower caloric load or a novel mouthfeel. \\ Enhancing the functionality of oil-continuous emulsions has traditionally been limited by the \\ dearth of oil-soluble emulsifiers that are available. More recently, efforts have focused on \\ engineering fat crystals as emulsion stabilizers. Fat may comprise a 3D network in the oil phase \\ that provides structure to the emulsion or a portion may reside at the interface of dispersed droplets. \\ It has been shown that structuring the droplet oil-water interface with adsorbed fat may have in an \\ influence on emulsion rheology. Explaining the rheological behaviour of such composite \\ emulsions relies on an understanding of the individual and synergistic contributions of fat crystals \\ and emulsions, where one draws inspiration from materials science, fat crystallization theory and \\ food science. Though fat crystallization increasingly forms part of the growing arsenal of tools to \\ modulate W/O emulsion functionality, much remains to be done in order to exploit this approach \\ as a bona fide emulsion structuring tool. This review explores recent findings in the field that \\ demonstrate the growing relevance of fat crystal-stabilized W/O emulsions for functional \\ applications and novel approaches to modifying their texture and rheology.
}




\subsection{Introduction}

Emulsions consist of two immiscible fluids that form a suspension with one phase dispersed as micron or nano-scale droplets in the other, which acts as the continuous phase. Oil-in-water $(\mathrm{O} / \mathrm{W})$ emulsions (dairy cream, dressings, etc.) are usually fluid whereas food-related water-in-oil (W/O) emulsions (e.g., butter and margarine) are oil-continuous and often solid-like. Though less common than their $\mathrm{O} / \mathrm{W}$ counterparts, there is growing interest in the use of $\mathrm{W} / \mathrm{O}$ emulsions for applications including encapsulation and delivery of volatile or labile ingredients (e.g., flavours, vitamins), masking of off-flavours (e.g., bitterness), prevention of rancidity, protection of bioactive compounds during gastrointestinal transit, and lower-calorie and reduced fat products where maintaining textures comparable to full-fat foods is key.

From a materials science viewpoint, emulsions are thermodynamically unstable composites where droplets act as a filler dispersed within a continuous phase matrix. In $\mathrm{O} / \mathrm{W}$ emulsions, addition of filler droplets to a continuous phase can give rise to dispersions with consistencies ranging from thin liquids such as vinaigrette to highly elastic solids such as self-supporting mayonnaise, depending on droplet packing, and interactions (Barnes, 2003, 1994; Perrechil et al., 2010; Sala et al., 2009). Yet, whereas many water-soluble emulsifiers, proteins or thickening gums are available to tune droplet interactions in $\mathrm{O} / \mathrm{W}$ emulsions, only a handful of oil-soluble emulsifiers and thickening agents exist for W/O emulsions. Conversely, in oil-continuous systems, solid fat and increasingly, oleogelation, are being considered as tuning parameters to potentially control texture and rheology.

The interactions between a filler material and continuous matrix will play a dominant role in the material properties of an emulsion, including the development and conservation of the type, 
magnitude, and stability of product texture as it ages. Interactions may be physical or chemical in nature and may be of the filler-filler, filler-matrix or matrix-matrix type. Such interactions, whether synergistic, antagonistic or neither, may occur at the molecular, supramolecular or microscale and vary according to composition, processing conditions, consumer handling and during ingestion.

In this chapter, we focus on the textural variables that define fat crystal-stabilized W/O emulsions. We show how fat crystal-stabilized W/O emulsions are best viewed as composites of two systems in one - a continuous phase fat crystal network and a dispersed aqueous phase -with each individually contributing to overall rheology, but importantly, also via their synergistic or antagonistic interactions. We also show that extrapolating from bulk fat rheology towards a composite system fails given the presence and role of a dispersed aqueous phase. We conclude by showing example of how interactions among ingredients can be used to manipulate the texture of fat crystal-stabilized W/O emulsions.

\subsection{Emulsions}

Emulsions are formed through the high energy mixing of one immiscible phase into another, thereby creating a thermodynamically unstable, ideally homogeneous dispersion of droplets of one phase within a continuous phase. Emulsions contain anywhere from $<1 \%$ to upwards of $75 \%$ of dispersed phase. The proportion of dispersed phase present within the continuous phase influences important characteristics including appearance, flavour, stability and texture (Tadros, 2013). Dispersed droplets (whether oil or water) range in diameter from less than $1 \mu \mathrm{m}$ to upwards of 100 $\mu \mathrm{m}$ and are typically polydisperse. 
The free energy upon emulsification $(\Delta G$, Equation 1.1) can be written as a function of the increased interfacial area $(\Delta A)$, interfacial tension $(\gamma)$, absolute temperature $(T)$, and configurational entropy $(\Delta S)$ (Tadros, 2013):

$$
\Delta G=\gamma \Delta A-T \Delta S
$$

Given the inherent interfacial tension between the two phases, the interfacial area term is always positive, meaning the overall free energy change is unfavourable. Therefore, emulsion instability arises from the thermodynamic drive to decrease interfacial surface area. It is the kinetic parameters of a system, however, that govern how quickly the emulsion breaks down and can be used to counter the thermodynamic drive to destabilize. It is in this regard that the presence, type and concentration of a stabilizing system is critical to emulsion functionality.

When mixed in the absence of surface-active agents (emulsifiers), the oil and aqueous phases within an emulsion will rapidly separate into their constituent phases. Phase separation can occur via creaming/sedimentation (separation of the droplet-rich phase to top/bottom of the mixture), flocculation (aggregation where droplets retain their individual identity), coalescence (the merging of flocculated droplets into larger droplets), partial coalescence (where partly crystalline dispersed oil droplets aggregate), and Ostwald ripening (formation of larger droplets as small droplets are re-adsorbed by the continuous phase) (Spyropoulos et al., 2011). Emulsifiers can improve miscibility of the dispersed phase and prevent destabilization. Steric barriers, such as fat crystal networks and interfacial crystals may also physically impede coalescence and flocculation/sedimentation processes. These will be discussed in following sections. 


\subsection{Emulsifiers}

The kinetic destabilization of emulsions can be slowed by the use of emulsifiers, which are amphiphilic molecular species that allow for the formation of nano- or micron-sized droplets within a continuous phase (McClements, 2005). Emulsifiers do so by lowering the interfacial tension between the two phases, allowing for the dispersion of an otherwise immiscible dispersed phase given their amphiphilicity, which renders them surface-active. As a result, they preferentially adsorb to the oil-water interface, with their polar groups oriented towards the aqueous droplet surface and nonpolar groups residing in the continuous oil phase.

Emulsifier solubility in either the aqueous or oil phase will dictate whether it promotes the formation of an $\mathrm{O} / \mathrm{W}$ or W/O emulsion, as semi-empirically described by its hydrophilic-lipophilic balance (HLB) value. HLB is a measure based on the relative contributions of hydrophilic and hydrophobic functional groups within a molecule. Emulsifiers that promote formation of W/O emulsions have low HLB values, meaning they are predominantly lipophilic, and vice-versa for O/W emulsions, as stipulated by Bancroft's rule (Tadros, 2013).

Compared to $\mathrm{O} / \mathrm{W}$ emulsions, where many water-soluble emulsifiers, proteins or thickening gums are available for emulsion formation and stabilization, only a handful of oil-soluble emulsifiers for W/O emulsions exist. In this regard, many efforts have focused on developing fat crystals as emulsion stabilizers, as discussed below. 


\subsection{Emulsion rheology}

The rheology of emulsions depends on key properties such as the oil:water ratio, composition of the dispersed and continuous phases, the type and concentration of emulsifier, processing conditions and storage (Barnes, 2003, 1994). These parameters will impact properties such as the interfacial tension between the phases, average dispersed phase droplet size and droplet size distribution as well as the viscosity of the emulsion (Barnes, 2003, 1994; Tadros, 2013). Ultimately, this will dictate the kinetic stability of the emulsion as well as its possible applications (Norton et al., 2009; Tadros, 2013).

Well-established models exist for modeling the relative viscosity of solid suspensions based on non-interacting hard sphere models. These have been applied to explain rheological phenomena of dispersed liquids i.e., emulsions with good agreement with experimental data (Farris, 1968; Genovese, 2012; Krieger, 1972; Luckham and Ukeje, 1999). The Einstein equation (Equation 1.2) describes the relative viscosity, $\eta_{r}$, of a solid suspension at low particle volume fractions:

$$
\eta_{r}=\eta_{m}(1+[\eta] \phi)
$$

Where $\eta_{m}$ is the viscosity of the surrounding medium, $[\eta]$ is the intrinsic viscosity of hard spheres (2.5 for rigid spheres), and $\phi$ is the volume fraction (Do et al., 2007; Genovese, 2012). While this model is effective at low particle concentrations $(\phi<0.1)$, the model fails to explain dispersion viscosity at higher particle loading. The Krieger-Dougherty model (Equation 1.3) provides better estimations of viscosity at higher particle volume fractions, up to approximately $60 \% \mathrm{w} / \mathrm{v}$.

$$
\eta_{r}=\left(1-\frac{\phi}{\varphi_{m}}\right)^{-[\eta] \varphi_{m}}
$$


As with the Einstein equation, $\eta_{r}, \phi$ and $[\eta]$ are the relative viscosity $\left(\eta_{\text {dispersion }} / \eta_{\text {continuous phase }}\right)$, the volume fraction and the intrinsic viscosity of the non-interacting particles. The maximum packing of particles $\left(\phi_{m}\right)$ is a theoretical estimate and represents a threshold value below which the particles do not interact and do not impart a change to the material's viscosity. Above this concentration, the particles begin to form a network that increases the material's viscosity and exhibits a yield stress (Genovese, 2012; Krieger, 1972; Luckham and Ukeje, 1999). Particle size is an important factor governing the viscosity of dispersions. The above models describe monomodal distributions; however, this is rarely the case in real systems where the particle size distributions are often multimodal. Particle size distributions can have varying effects on the viscosity of a dispersion. Assuming that the particles are non-interacting, an effective diameter of greater than 1/10 the size of the large particle component will impose an increase on the viscosity of the suspension. For a multimodal distribution, the relative viscosity of a suspension (Equation 1.4) is the product of the relative viscosity of each particle size distribution, $\eta_{r i} \phi_{i}$ (Farris, 1968).

$$
\eta_{r}=\prod_{i=1}^{n} \eta_{r_{i}} \phi_{i}
$$

Essentially, widening or narrowing the difference between particle size distributions can reduce or increase the relative viscosity of a suspension, respectively (Genovese, 2012). This model has good agreement with experimental viscosity values at high particle loading concentrations, however, it is only applicable to non-interacting spheres. For particles that display attractive interparticle interaction, the model fails to predict relative viscosity trends.

Since the changes in emulsion rheology are analogous to those caused by interparticle interactions of hard spheres and the same models are used to describe their rheology (Tadros, 2013), the same forces governing attractive forces between particles are those acting on dispersed droplets in an 
emulsion (hydrodynamic/fluid resistance forces, colloidal forces, electrostatic, hydrophobic interactions, etc.). These interparticle forces influence the overall behaviour of emulsions under shearing conditions (Barnes, 2003). As well, changes in surface area, particle size and volume fraction can impose similar trends. As such droplets can be broadly considered as particle suspensions whose rheology is determined by the number and strength of dispersed particle interactions, however, less quantitatively understood than their solid particle counterparts (Barnes, 1994).

Both oil and water demonstrate Newtonian flow behaviour with a linear relationship between shear stress and rate of shear strain (i.e. shear rate), where the proportionality constant relating these is the viscosity of the fluid. Incorporation of a dispersed phase generally increases viscosity. In the simplest case, non-interacting droplets will cause the emulsion viscosity to increase at low shear rates. This is due to the droplets moving within the continuous phase with applied shear, imposing local changes in flow adjacent to the droplet (Chen et al., 2010). At higher volume fractions, the dispersed phase can cause crowding, leading to further increases in viscosity until the dispersion flows no longer and transitions to a predominantly elastic material. This trend towards thickening can be compounded by droplet flocculation, which implies that droplet interactions play a role in this regard. At high shear rates, viscosity can increase or decrease, known as shear-thickening or shear-thinning, depending on the attractive forces between droplets and their ability to resist shear deformation (Barnes, 1994; Chen et al., 2010).

W/O emulsion rheology depends droplet size and distribution, apparent and real volume fraction, deformability and tendency to aggregate (Barnes, 1994). The presence of dispersed aqueous droplets strongly impacts rheology, in particular at higher volume fractions where large increases 
in emulsion rigidity result (Frasch-Melnik et al., 2010; Haj-shafiei et al., 2013; Johansson and Bergenståhl, 1995; Lopes da Silva and Coutinho, 2004; Macierzanka et al., 2009).

\subsection{Fat crystallization}

Fat largely consists of triacylglycerols (TAGs), which are made up of three fatty acid chains (acyl groups) esterified to a glycerol core (Lawler and Dimick, 2002). Crystallization in bulk fat has been well-characterized and it is understood that the textural attributes of fat crystal network depend on many compositional, processing and storage variables. The TAG composition of natural fats is highly variable, which will greatly impact crystallization behaviour. For example, cocoa butter consists of 3-4 key TAGs whereas milk fat contains hundreds of TAGs capable of influencing crystallization behaviour. This compositional variability can result in complex crystallization behaviour (Sato et al., 2013).

The degree of unsaturation, chain length of the constituent fatty acids, and their relative position on the glycerol backbone ultimately determine how TAG molecules pack in a 2D plane and stack lengthwise. Saturated fats have higher melting points than unsaturated. The presence of an unsaturated fatty acid can affect polymorphic transitions depending on which of the 3 positions it occupies on the glycerol backbone (sn-1, sn-2, or sn-3). The TAGs adopt $\beta^{\prime}$ or $\beta$ polymorphs with 2 or 3 chain length stacking to place the unsaturated fatty acid away from the saturated tails. (Himawan et al., 2007, 2006).

TAGs may assume different polymorphs and exhibit different crystal sizes under different cooling rates. Fast cooling rates under static conditions have been associated with smaller crystal size and 
less stable polymorphs. Slower cooling rates result in more stable polymorphs and a larger crystal size. Different polymorphs may exhibit different perceived hardness, an important factor for sensory qualities of food products. For example, creaminess of margarine is attributed to the $\beta^{\prime}$ polymorph, whereas grittier texture is associated with the more stable $\beta$ form (Miskandar et al., 2005).

Control of the liquid-solid transition in such TAGs is crucial for the proper development of microstructure and texture in many foods. Crystallization of fat from the molten liquid phase requires sufficient supercooling of a melt to form nuclei, which are the smallest-sized entities able to withstand resolubilization at a given temperature and concentration. Growth into crystals is initiated when molecules assemble on the surface of nuclei, leading to the formation of larger and larger crystals (de Man and Beers, 1988; Lawler and Dimick, 2002).

Fat nucleation occurs via one of two modes - homogeneous and heterogeneous. The latter occurs on a foreign surface associated with the melt at a given temperature, e.g., higher-melting crystal seeds, emulsifiers, container surface irregularities and importantly, dispersed droplet surfaces. As homogeneous nucleation occurs in the absence of impurities, it directly takes place from the melt when two or more TAG molecules come into close contact and adopt an ordered conformation. This newly-formed nucleus recruits adjacent TAG molecules to facilitate crystal growth. Both modes of nucleation are affected by the rate of supercooling where higher rates lead to smallerdimension crystals, whereas slower cooling rates tend towards larger crystals (Campos et al., 2002; Maleky et al., 2012). While the crystals formed under fast supercooling conditions are smaller, the crystals themselves have a less ordered crystal structure than those formed using slower supercooling rates (Lawler and Dimick, 2002; Sato et al., 2013). 
Fat crystal networks are defined as assemblies of crystals that hierarchically associate into a spacefilling crystal network. The first level of organization consists of TAG forming supramolecular assemblies ("nuclei") result in first level of organization. These then aggregate to form micro-scale crystal clusters, which further aggregate to form an interconnected crystal network. The rheological properties of the crystal network depend on TAG composition, fat crystal size, polymorph, aggregation state and particle-particle interactions. The structure and rheological properties of this network are responsible for the mechanical strength and yielding, which may confer a solid-like character with a degree of plasticity to macroscopic bulk fats (Marangoni et al., 2012).

\subsection{Rheology of fat crystal networks}

The rheology of fat crystal networks depends on properties that include the fatty acid composition and their positional distribution (Himawan et al., 2006), the type and concentration of added emulsifier and other ingredients, processing conditions and storage (Garbolino and Bartoccini, 2005; Herrera and Hartel, 2000; Johansson and Bergenståhl, 1992a; Smith et al., 2011), which ultimately define properties such as solid fat content, TAG crystallization kinetics, fat crystal size and polymorphic behaviour, extent of aggregation as well as small (e.g., viscoelasticity) and large deformation (e.g., yielding) behaviour (Garbolino and Bartoccini, 2005; Gregersen et al., 2017; Herrera and Hartel, 2000; Johansson and Bergenståhl, 1992b; Ramamirtham et al., 2017; Smith et al., 2011; Thareja, 2013).

Fat crystal networks are a collection of crystalline aggregates held together by van der Waals forces (Piska et al., 2006). These bonds can stretch upon the application of stress, causing deformation 
of the network until a yield point is reached. The system then behaves as a viscous liquid and permanent deformation of the network may occur (de Man and Beers, 1988). Many factors affect rheological properties of a fat crystal network. Fat crystal size and polymorphism both play a significant role on kinetic stability. Smaller fat crystals increase the number of aggregate interactions, which can lead to higher structural rigidity (de Man and Beers, 1988), which reduces network porosity and strengthens the crystal lattice (Lopes da Silva and Coutinho, 2004). Age also plays a significant role, as fat crystals sinter with time. For example, hydrogenated palm oil-palm oil blends increased in hardness after ageing 28 days, implying that the crystals in the network continued to grow over time after initial solidification (Omar et al., 2005).

The effect of solid fat content on elastic modulus is well-known in bulk fat systems. It has been shown experimentally that dependency is not linear, implying the presence of other synergistic factors (Vreeker et al, 1992). While the strength of particle-particle interactions and particle volume fraction are key factors in determining overall viscoelastic character, they are not comprehensive. Therefore, crystal aggregate structure has been investigated as a determinant factor in fat crystal network rheology (Campos et al., 2002; Maleky et al., 2012; Maleky and Marangoni, 2011; Marangoni et al., 2012; Shih et al., 1990). Fractality is "self-similarity" at different length scales e.g. a single structure at different magnifications look somewhat similar (Marangoni and Rousseau, 1996; Narine and Marangoni, 1999; Shih et al., 1990). Fractal flocs, such as fat crystal aggregates, can have numeric values ascribed to them based on light scattering that describe their fractal growth (Vreeker et al., 1992). With regards to fats, the rheology of high concentration gels (high SFC) may be explained by the weak-link theory, where the links between neighbouring fat crystal flocs have a smaller elasticity than those within the flocs themselves. Conversely, the rheology of low concentration gels (i.e.,, low SFC systems) may be explained by 
the strong-link theory, where the links between neighbouring flocs have a larger elasticity than those in the flocs (Narine and Marangoni, 1999; Shih et al., 1990). How the elasticity of strong and weak links in the crystal network is affected by the presence of a dispersed aqueous phase is currently unknown, however. Clearly, diffusional processes governing crystal aggregation differ as available space is partly occupied by water droplets.

\subsection{Emulsifiers as fat crystallization modifiers}

Emulsifiers may significantly impact TAG nucleation, crystal growth or both, depending on their composition, added amount and mechanism of action (Smith et al., 2011). Addition of certain emulsifiers has been shown to mitigate fat crystallization. For example, sorbitan monostearate has been observed to inhibit chocolate fat bloom (Lawler and Dimick, 2002) and alter the crystallization of tristearin (Aronhime et al., 1988). The opposite effect has been observed as well with different emulsifiers where the additives act as crystallization "seeds" to accelerate crystallization and/or alter polymorphic behaviour (Lawler and Dimick, 2002; Thareja et al., 2012).

The presence of emulsifiers can induce rheological changes by reducing or increasing the strength of the interactions between the crystals comprising a network. For example, in the case of a fine tristearin crystal network, the presence of unsaturated monoglycerides (i.e., glycerol monooleate) at high concentration $\left(12.0 \%\right.$ ) decreased $\mathrm{G}^{\prime}$ from $10 \mathrm{kPa}$ to $9 \mathrm{kPa}$ as well as yield stress from 50 to $27 \mathrm{~Pa}$ compared to the emulsifier-free network at $20^{\circ} \mathrm{C}$, whereas low concentrations $(2.5 \%)$ showed little effect (Johansson and Bergenståhl, 1992b). Saturated monoglycerides (glycerol monostearate and monopalmitate) decreased $\mathrm{G}^{\prime}$ from 10 to $8.5 \mathrm{kPa}$ and yield stress from 50 to 42.5 
$\mathrm{Pa}$ at low concentrations $(2.0 \%)$, but had the opposite effect at high emulsifier concentrations (12.0\%) increased $\mathrm{G}^{\prime}$ and yield stress to $11 \mathrm{kPa}$ and $66 \mathrm{~Pa}$ respectively. At low concentrations, the emulsifier molecules adsorbed to the fat crystal surface, lowering the interaction strength between fat crystal aggregates. Increasing saturated monoglyceride content also increased the solid fat content, thus increasing $G^{\prime}$ and viscosity (Johansson and Bergenståhl, 1992a). These results suggested that the attractive forces between the emulsifier tail and TAGs were an important contributor to fat crystal network viscoelasticity. Hence, in bulk fats, emulsifiers may be used as a tool to modulate texture and rheology.

\subsection{Role of fat crystals in stabilization of W/O emulsions}

Foods such as butter, margarine, whipped cream and ice cream are either partially or wholly stabilized by surface-active fat crystals present at the water/oil interface. Such solid particles, known as 'Pickering' crystals, create a steric barrier between adjacent water droplets thereby hindering droplet collisions, film drainage and coalescence. If fat crystals are surface-inactive,

their propensity to reside at the interface will be greatly diminished. Such crystals are more apt to interact via van der Waals interactions and form a fat crystal network that stabilizes W/O emulsions not by interfacial adsorption, but by physically encasing the dispersed phase. Finally, fat crystals may act as both Pickering and network stabilizers, depending on the presence of an appropriate surfactant that yield crystals with 'switchable' polarity. 


\subsubsection{Pickering particles for emulsion stabilization}

Given their exceptional capacity to kinetically stabilize emulsions, particle-stabilized emulsions have seen dedicated research efforts both in industrial and academic circles since the 1990s, whether for applications in personal care products, drug delivery, and processed foods, where particles present as wax or fat crystals, silica, clay, or polymers adsorbed to the oil-water interface effectively hinder dispersed phase thin film drainage and coalescence by creating a mechanical barrier between adjacent water droplets (Chevalier and Bolzinger, 2013; Melle et al., 2005; Tavernier et al., 2016). In foods, fat crystal-based particles may consist of TAGs or emulsifier crystals and originate via direct solidification at the interface (Krog and Larsson, 1992) and/or absorption of previously-formed crystals on the droplet surface (Garti et al., 1998). Pickeringstabilized emulsions are formed by these particles adsorbing to the droplet interface, forming a diffuse monolayer surrounding a dispersed droplet. This provides a mechanically stable steric barrier against droplet coalescence (Aveyard et al., 2003; Binks, 2002; Melle et al., 2005).

\subsubsection{Core-shell particle emulsion stabilization}

A prerequisite for effective emulsification is the presence of an appropriate emulsifier that results in an appropriate droplet size. Emulsifier adsorbed onto the surface of the water droplet surface may then potentially act as a substrate to promote adsorption of TAG crystals, however this will depend on several factors, including emulsifier properties, physical state and concentration.

Emulsifiers play a significant role in tuning fat crystals towards bulk network or interfacial crystallization. For example, petroleum wax crystals are not inherently interfacially-active, given 
their alkane composition and preferentially reside in the continuous oil phase. Wax-in-oil systems will gel as the wax crystallizes and aggregates. Wax crystals follow similar trends as fat where many small crystals will form more rigid structures than larger, but fewer aggregates (Lee et al., 2008; Vignati et al., 2005). In W/O emulsions where wax is the solid crystal species, it has been shown that the presence of surfactant can promote adsorption of wax crystals to the water droplet interface and can increase the apparent viscosity compared to both surfactant-stabilized, wax-free

emulsions and wax-containing, surfactant-free emulsions. Apparent viscosity is higher than both these systems when both surfactant and wax are present (Ma et al., 2017). This increase in viscosity is due to gelation which is enhanced when the surfactant promotes the adsorption of wax to the droplet interface and thus increasing the number and size of droplet aggregates (Visintin et al., 2008, 2005). This increase in viscosity can be enhanced by increasing the water concentration which promotes aggregation behaviour of wax stabilized droplets (Haj-shafiei et al., 2013; Visintin et al., 2008).

\subsection{Rheology of fat crystal-stabilized W/O emulsions}

The presence of dispersed droplets can alter the rheology, crystallization kinetics and morphology of fat crystal networks. For example, water droplets hasten the crystallization of cocoa butter versus bulk cocoa butter and promote the formation of the most stable form V polymorph. (Di Bari et al., 2017). It was postulated that the ordering created by the water-cocoa butter interface provided "facilitated TAG structural re-arrangement". This was further supported by the observation that no such increase in crystallization rate or tendency to form $\mathrm{V}$ was seen in the 
presence of PGPR, a branched polymeric surfactant. This was attributed to the less organized molecular arrangement of PGPR, preventing polymorphic evolution.

How dispersed droplets are stabilized will also impact the stability of an emulsion against phase separation. For example, Hodge and Rousseau found that emulsions containing a fat-crystal network comprised of hydrogenated canola oil yielded Pickering-stabilized water droplets that exhibited the highest degree of stabilization in quench cooled samples after emulsification with the highest solid fat content providing crystal network stabilization (Hodge and Rousseau, 2005). Studies have been done to elucidate the effect of incorporating a dispersed water phase on the viscoelastic properties of a crystal network. Lopes da Silva and Coutinho have shown that in wax crystal-stabilized gels, the network strength is proportional to wax content as well as the oil phase chain length where oils with higher degrees of saturation display gels of higher rigidity (Lopes da Silva and Coutinho, 2004). As well, fast-cooling produced a gel-like solid containing small, aggregated spherulitic clusters, whereas slow cooling may result in solid precipitation and larger crystal formation. This is in keeping with the crystal network stabilization study performed by Hodge and Rousseau.

Similar to bulk fat crystal networks, physical ageing of the continuous fat crystal network stabilized W/O emulsions will also greatly affect rheology, and resistance against coalescence and water separation, as has been observed in previous studies using paraffin wax crystals as the network crystal (Haj-shafiei et al., 2013; Macierzanka et al., 2009). This results in a more rigid overall structure and it is expected to see the trend continue with age. As they age, fat crystals may grow further and enmesh or sinter resulting in a material that is stiffer and more brittle. Hence, long-term emulsion rheology and stability will hinge on how nascent and existing crystals grow and aggregate, which will greatly depend on composition (TAG make-up, emulsifiers, etc.), 
processing (temperature, shear) and storage conditions as well as interparticle interactions (e.g., van der Waals forces, sintered solid crystal bridges, etc.). The presence of emulsifiers potentially alter the strength of particle-particle interactions (Johansson and Bergenståhl, 1992a; Marangoni and Rousseau, 1996; Narine and Marangoni, 1999). In emulsions, emulsifiers at the interface of a water droplet can also bring about rheological changes that are significantly different than emulsifiers present in a water-free bulk fat crystal network.

\subsection{Droplets as active fillers in emulsions}

The concept of fillers, a foundational tool in materials science, has been extensively applied to water-continuous foods, namely $\mathrm{O} / \mathrm{W}$ emulsions gels, with the general agreement that the interface plays a part in strengthening/weakening material structure (Chen and Dickinson, 1999; Dickinson and Hong, 1995; Van Aken et al., 2015; van Vliet, 1988). Composite materials consist of two or more materials that, when combined, give a mixture with different rheological and mechanical properties than its constituent parts - the matrix, comprising the continuous network, and the filler material, the species embedded within the matrix (Ring and Stainsby, 1982). Fillers fall under two categories - active and inactive. Active fillers interact strongly with the surrounding matrix and impact the rigidity and deformation of the composite whereas inactive fillers show little or no interactions with the matrix and do not significantly alter composite rheology (Barnes, 2003; Fröhlich et al., 2005; McClements et al., 1993; Ring and Stainsby, 1982; van Vliet, 1988). Filler activity depends on its surface activity, particle size distribution, extent of aggregation within the continuous phase and volume fraction (Anton et al., 2001; Dickinson and Hong, 1995; Fröhlich et al., 2005). Emulsions show similar dependencies on these factors for their viscoelastic behaviour. 
Solid filler particles have their surfaces exposed to the surrounding matrix. Surface activity is largely dictated by the functional groups available on the surface to interact with the surrounding matrix and adjacent filler particles. In food systems, emulsifiers can alter the viscoelasticity of complex mixtures containing solid particles, resembling composite materials. Chocolate, for example, consists of sugar and cocoa particles suspended within a fat crystal network of solidified cocoa butter. The addition of emulsifiers, notably lecithin and polyglycerol polyricinoleate, can reduce the Casson yield value (force required to initiate flow) and lower apparent viscosity by coating the sugar surface, altering the strength of the interactions between the solid particles and the fat crystal matrix (Afoakwa et al., 2008; Middendorf et al., 2015).

Surface activity caused by the nature of the functional groups on the filler surface can improve the strength and number of contact points with the surrounding continuous phase matrix and as such, increase the level of reinforcement under shear (Dickinson and Chen, 1999). For example, heat set emulsion gels exhibit higher values of $\mathrm{G}^{\prime}$ when whey protein is used as an emulsifier. A non-ionic surfactant, such as Tween 20, does not form covalent bonds as some proteins do upon heating. In this case, amino acid functional groups present on the droplet surface create covalent bonds with the surrounding matrix and adjacent droplets during processing. As well, some proteins can form strong physical bonds (van der Waals, hydrogen bonding etc) which leads to aggregation and subsequent reinforcement compared to the unfilled matrix (Chen and Dickinson, 1999; Dickinson and Chen, 1999). Such examples show that filler surface activity is a reflection of filler-filler interactions, as well as those with the surrounding network.

Particle size and volume fraction are other variables with a significant influence on filler activity. The size of the particles and the loading concentration ultimately alter the number of contact points a matrix can make (e.g., crosslinks in a fibrillar network and physical contacts within a particle 
matrix). Higher concentrations (i.e.,, particle loading) of an active filler can substantially reinforce the material, while increasing concentrations of an inactive filler may have nil effect or break matrix structure (Fröhlich et al., 2005). In solid particle-fat crystal network systems, such as chocolate, small particle sizes increase Casson plastic viscosity. This reduction is proportional to the number of attractive physical interactions (non-crosslinking) between particles and their surroundings (Afoakwa et al., 2008; Van Aken et al., 2015).

Aggregation or clustering of particles can reinforce composite materials as well. By increasing the number and strength of filler-filler interactions, the overall contributions of the filler component to reinforcement is increased. This ultimately depends on the strength of the filler-filler interactions within the aggregate (Fröhlich et al., 2005). It is important to note that while small active filler particle sizes tend to reinforce more effectively than larger particles, large aggregates of small particles can reinforce the material even further. Strong intra-aggregate interactions require greater shear in order to destabilize the aggregate. These interactions are different than those between the filler particles and the matrix but can contribute to the overall viscoelastic behaviour of the material. In chocolate, low fat content increases the amount of aggregation between suspended solids. This causes heightened particle interaction, which form aggregates that result in a hard final product (Do et al., 2007). In composite hydrocolloid gels, increasing the volume fraction of the dispersed particles leads to clustering, with the individual clusters growing more dense with increasing particle concentration. Further increasing the particle concentration causes particle clusters to form cluster aggregates, behaving as large deformable particles. The deformation tendency of these cluster aggregates varies depending on the strength of interparticle interactions. The strength of these interparticle interactions given volume fraction ultimately dictates how effective the particles reinforce the material in comparison to the unfilled matrix (Van 
Aken et al., 2015). Bimodal particle size distributions can be used to optimize the contributions of the fillers to achieve the desired flow behaviour of the final product by altering the packing of the aggregates. Small particles fill the interstices within larger particle aggregates to increase the overall number of associations to make a more compact, harder aggregate cluster (Servais et al., 2002). The key types of interactions between filler particles and their surrounding matrix are depicted in Figure 1.1.
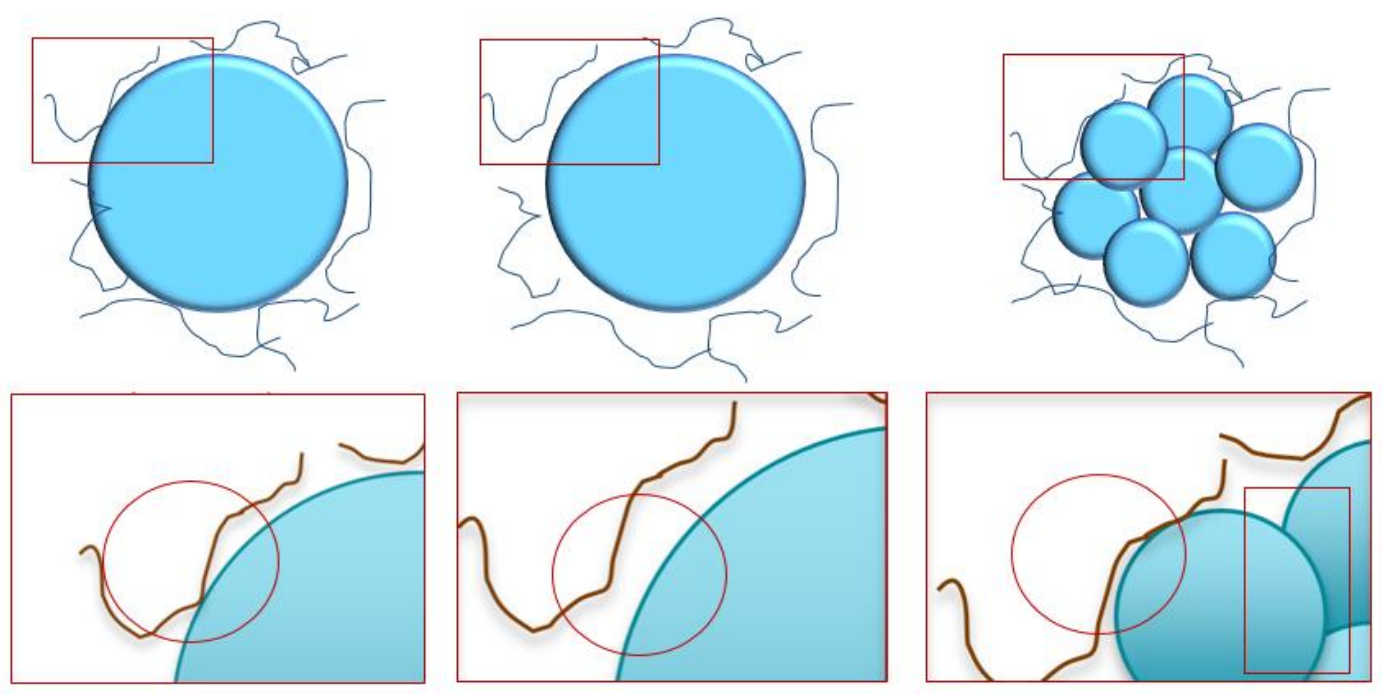

Figure 1.1: Interactions between a filled gel matrix and filler particles. Top row shows the type of interaction associated with each filler type. The bottom row shows the boxed area from above picture. Left - Active filler: Circle shows the close proximity of the filler surface to the matrix, resulting in the stronger interactions that cause reinforcing. Middle - Inactive filler: Circle shows the lack of interaction between the filler and matrix. Right - Aggregated filler: Rectangle shows filler-filler interaction, circle shows filler-matrix interactions (adapted from van Vliet (1988).

The behaviour observed in solid particle composites can be extended to dispersed liquid phases. As the packing fraction of dispersed water droplets heavily depends on water load, droplet flocculation will alter the apparent volume fraction and droplet size. For example, if interactions result in floc formation, water droplets no longer behave solely as discrete particles that interact 
with the surrounding matrix, but as an aggregate mass of droplets. The strength of the droplet aggregates then confers enhanced viscosity and viscoelastic properties, such as $\mathrm{G}^{\prime}$, to the overall emulsion. In the case where irreversibly-attached fat crystals interact to transform the interface from fluid to solid (and thus possesses an elastic modulus), the viscoelastic properties of the resulting interface will strongly depend on the interaction between the particles. Strong interactions and crystal-crystal sintering at the interface will impact deformation whereupon a significant force will be required to lead to droplet shape buckling and breakdown. If the interface remains fluidlike, deformation may permit a higher droplet packing density, and impact the maximum aqueous phase volume possible.

\subsection{Droplets as active fillers in W/O emulsions within a fat crystal network}

Since the dispersed aqueous phase is a key contributor to overall W/O emulsion rheology, simply extrapolating the rheological behaviour of bulk fats to emulsions is not appropriate (Haj-shafiei $e t$ al., 2013; Visintin et al., 2005). The dispersed aqueous phase of a W/O emulsion may interact strongly with the surrounding fat crystal network thereby resulting in emulsions with tunable rigidity, deformation, and storage stability simply by tailoring fat crystal-droplet interaction. As such, dispersed water droplets may be considered as a functional component of fat crystal networkstabilized W/O emulsions that can be used to tailor rheology. Treating droplets as particles with functional surfaces rather than just the dispersed phase allows us to build on the number of variables that we can control that goes beyond simply solid fat content and bulk fat properties.

Recent efforts have shown that modulating the interactions between fat crystals and water droplets offers the possibility of tailoring W/O emulsion rheology. For example, borrowing a concept used 
in $\mathrm{O} / \mathrm{W}$ emulsion gels where the dispersed oil phase may interact strongly with the surrounding gel matrix ('active filler') or show little to no interaction ('inactive filler') (Fröhlich et al., 2005; Genovese, 2012; McClements et al., 1993; Ring and Stainsby, 1982; van Vliet, 1988), it was recently shown that $\mathrm{W} / \mathrm{O}$ emulsion rigidity was enhanced when emulsion droplets interacted with surrounding fat crystals - here, droplets acted as an active filler. Conversely, weak droplet interactions with fat crystals did not alter emulsion rheology, hence these dispersed droplets were inactive fillers. Emulsifier type was the dominant factor resulting in droplets behaving as active fillers or not (Rafanan and Rousseau, 2017).

Figure 1.2A shows the different factors associated with the dispersed droplets which are thought to affect the rheology of fat crystal-stabilized W/O emulsions. In TAG-oil bulk crystal networks (Figure 1.2B), factors dictating structural rigidity are triglyceride molecular composition (fatty acid chain spatial distribution, saturation, TAG chain length etc), solid fat content, polymorphic form, crystal size, and fractal dimensions. In networks containing non-fat solids or emulsifiers, impurity characteristics affects the structural behaviour by altering the attractive interactions between fat crystals and aggregates. Emulsion droplets in the absence of a network have similar factors affecting droplet-droplet interactions associated with attractive-repulsive forces associated with the interface, which can be manipulated through controlling emulsifier characteristics. Factors controlling fat crystal network-stabilized emulsion rheology, a combination of the two different systems, are outlined in Figure 1.2C. Those common to both fat crystal networks and composite materials are shown in grey. Factors common to emulsions and composites are shown with dotted border. Factors controlling fat crystal network-stabilized emulsion rheology that coincide with factors dictating composite rheology are shown in black. It is seen here that fat crystal network stabilized emulsion interactions and composite material interactions often overlap. 
This involves considering water droplets within fat crystal network-stabilized emulsions as functional participants in network rheology, giving greater significance to the role of the interactions of the droplet interface.

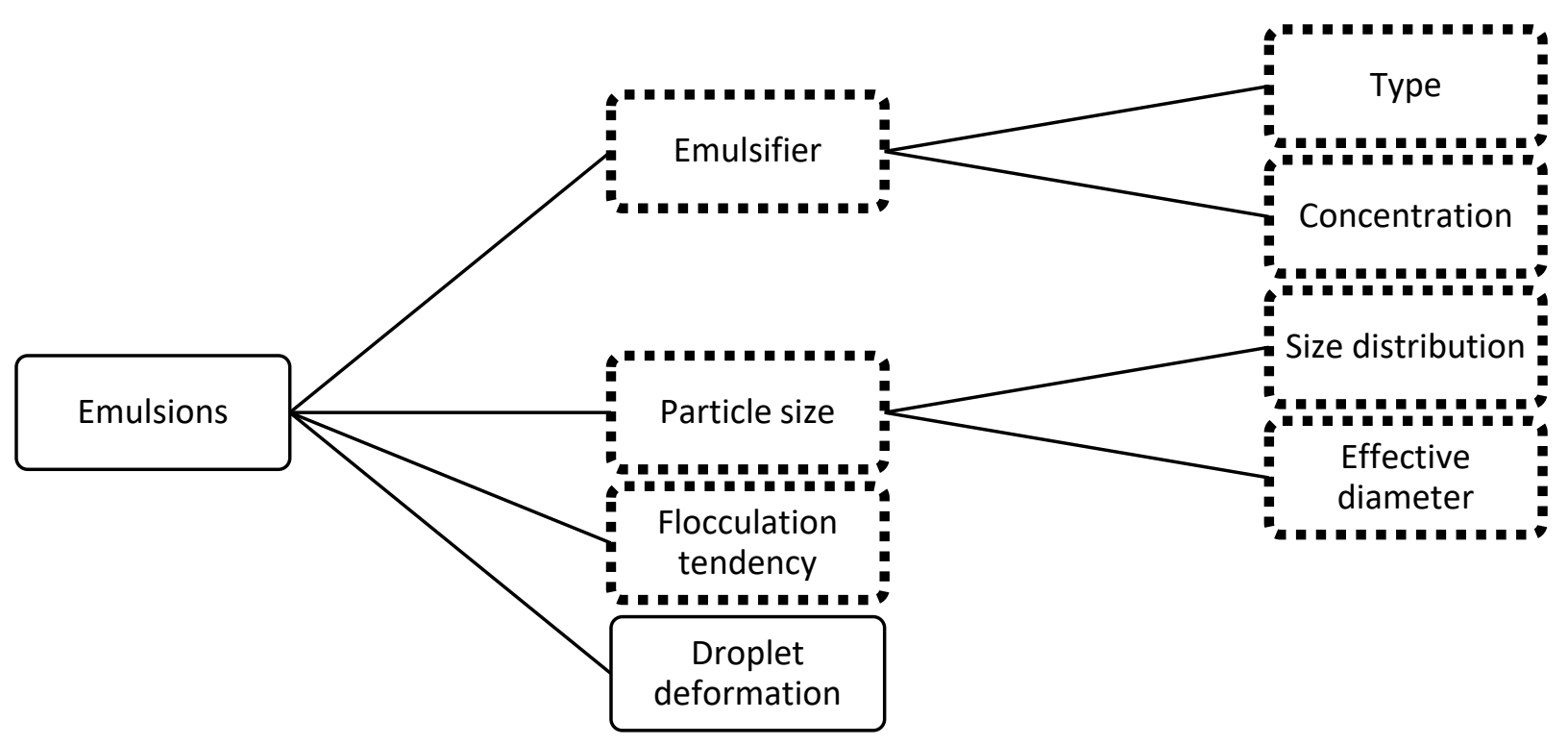

Figure 1.2A - Major factors dictating rheology in emulsions. The dotted, bordered boxed show common elements to composite rheology and emulsions. 


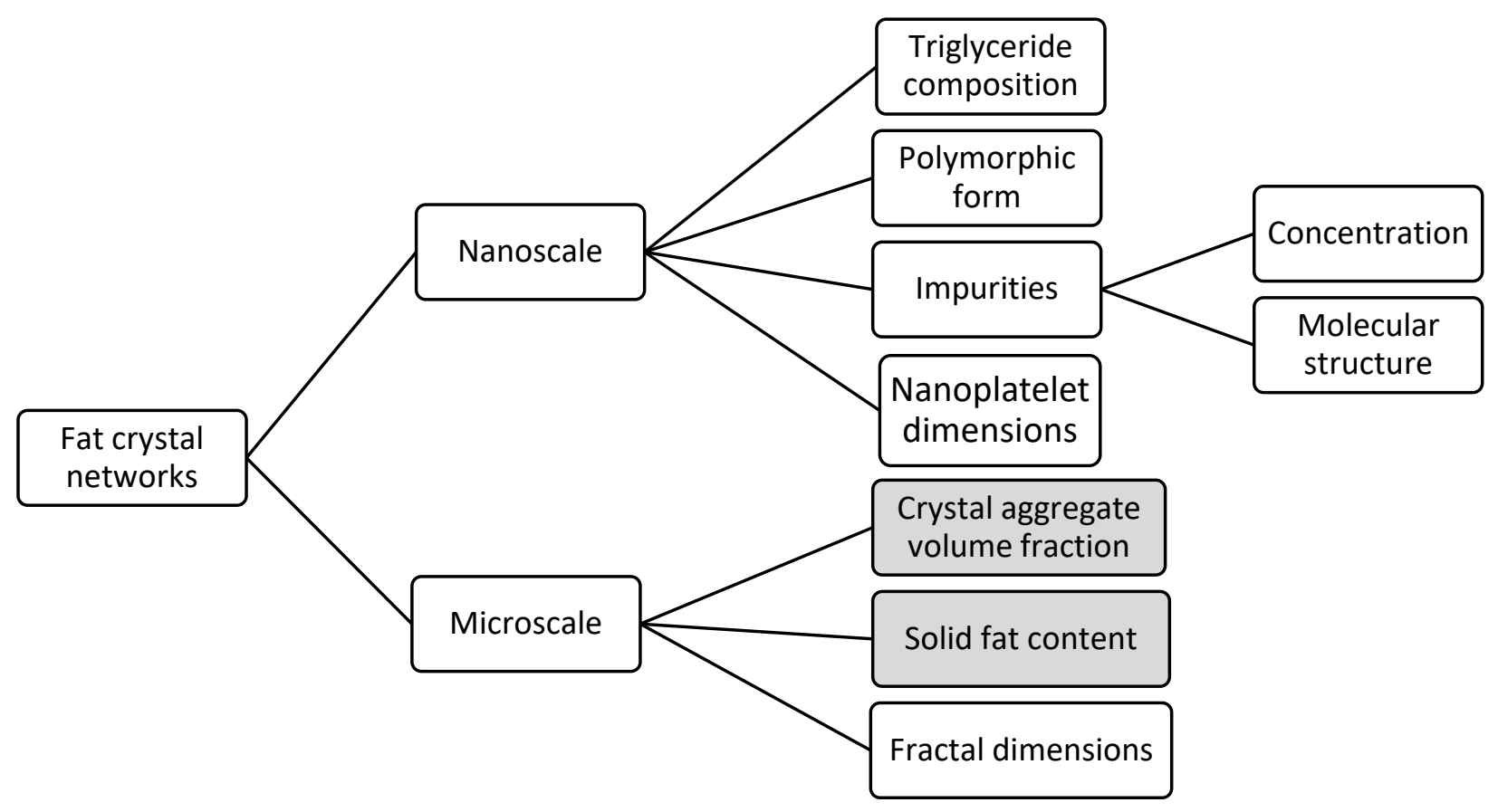

Figure 1.2B - Major factors dictating rheology in fat crystal networks. The grey boxes show variables common to fat crystal network rheology and composite materials. 


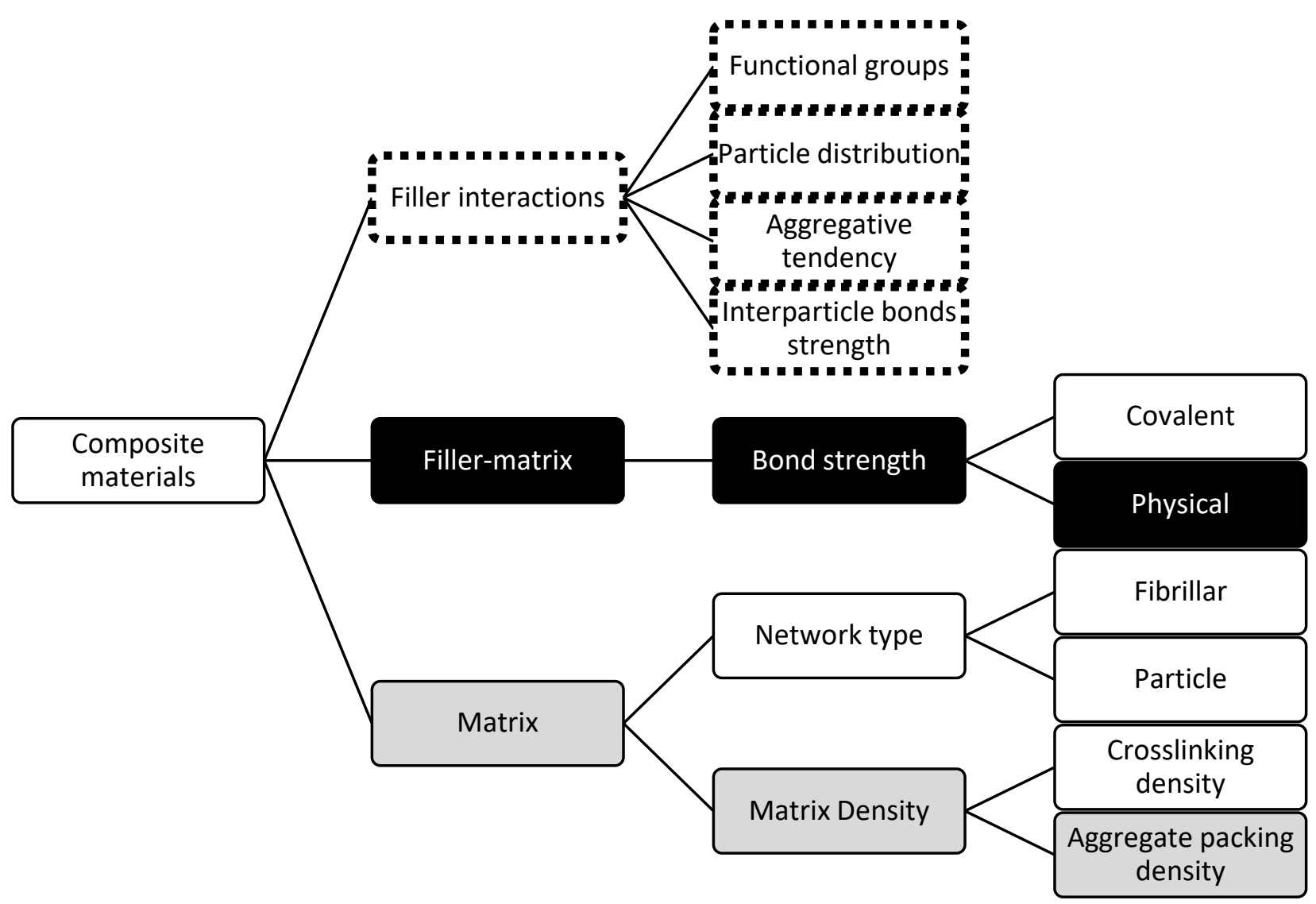

Figure 1.2C - Major factors dictating rheology in composite systems to show the intersectionality of structural interactions. The grey boxes show variables common to fat crystal network rheology and composite materials. The dotted, bordered boxed show common elements to composite rheology and emulsions. Black boxes in Figure $\mathrm{C}$ show the areas that can be applied to fat crystal network-stabilized emulsion rheology specifically.

In addition to possibly altering crystallization of the fat crystal network itself (Aronhime $e t$ al., 1988), emulsifiers can affect overall viscoelastic properties when present as a stabilizing film surrounding a droplet after the network has been established. As well as promoting network structure formation during the crystallization process, the emulsion droplets themselves may act as fillers after the network has formed. This effect has been observed in gel systems where simple replacement of the surfactant had a marked effect on rheology (Chen and Dickinson, 1995a; 
McClements et al., 1993). If the rigidity of the filler is greater than that of the matrix and there is good physical bonding between the particles and its surrounding network, the filler will have a reinforcing effect on the gel (Genovese et al., 2010). As well, if the interaction between the surface of the filler is strong, there is a palpable increase in system rigidity, as evidenced by an increase in G' (Chen and Dickinson, 1995b; Dickinson and Hong, 1995). Similar effects on crystal network rheology in the presence of fillers is expected, but research specifically addressing surface qualities and other filler characteristics are sparse.

For example, Chen and Dickinson showed that emulsion droplets coated with surfactants altered the elastic modulus of a heat-set whey protein gel (Chen and Dickinson, 1999). The active filler, oil droplets stabilized only by the whey protein, dramatically increased $\mathrm{G}^{\prime}$ compared to the dropletfree gel. Tween 20 stabilized droplets (inactive filler) required higher whey protein concentration to form a gel network of similar rigidity to the emulsion gel containing an active filler. For these gels, the increase in rigidity was attributed to the formation of disulfide bonds in the presence of the active filler upon heating, which was absent in the Tween 20-stabilized emulsion gel. However, Tween 20 at low concentrations was shown to have the opposite increase $\mathrm{G}^{\prime}$ on whey protein gels if the surfactant was added after homogenization, but before heat setting (Dickinson and Hong, 1995). Hence, both emulsifier and processing conditions dramatically alter change the role a particular filler may have on its surrounding matrix.

Thus far, most filler-matrix studies have concentrated on classical solid particulate-matrix and gel systems, with crystal networks studied to a far lesser degree. We have shown that fat crystal network-stabilized emulsions containing droplets exhibiting different interfaces (liquid-state small molecule emulsifier vs. interfacial crystallization) can have very different $G^{\prime}$ values (Rafanan and Rousseau, 2017). It was shown that the small molecule surfactant stabilized emulsion did not 
exhibit a change in $\mathrm{G}^{\prime}$ despite increasing water content. However, the emulsions stabilized with interfacial crystals, displayed increasing $\mathrm{G}^{\prime}$ values with increasing water content. This shows that the interface is a functional space and can have dramatic effects on reinforcement. It is apparent that similarities between interfacial functional groups and the outer matrix is determinant in rheological behaviour.

\subsection{Applications of fat crystal-stabilized W/O emulsions}

Structured emulsions are seeing explosive growth in food product development and applications. Fat crystal-stabilized W/O emulsions are sought-after for applications that demand calorie or fat reduction and costs savings while maintaining similar textural and sensory attributes as their fullfat, higher-cost counterparts. For example, butter, margarine and other fat-based tablespreads can benefit from the reduction in fat while maintaining desirable texture by systematic emulsifier selection, as found in recent commercially-produced margarines where emulsifiers such as glycerol monooleate may be used to promote interfacial crystallization rather than using

hydrocolloid thickeners. Similarly, in confectionery fillings such as ganache, the quantity of costly ingredients such as cocoa-based products may be reduced by promoting particle-particle interactions via the use of appropriate emulsifiers (Dias et al., 2015).

Control of sensory properties in manufactured food products is essential to the commercial success of the final product. The ability to manipulate parameters such as creaminess, thickness and spreadability are essential for creating desirable products, but also functional products. For example, reduced-fat spreads are more healthful, but have similar organoleptic properties as their full-fat counterpart. Not only is consumer perception an important factor in developing these 
products, but also cost of production. Low-fat spreads, where higher volumes of water are incorporated into the fat-crystal network not only replaces some of the fat to reduce caloric content, but also replaces the more costly material (fat and oil) with water. Discovering how to exploit W/O emulsion droplets to deliver the expected product at a fraction of the cost is highly advantageous.

Reducing the fat content of food products such as bakery goods relies on final texture and rheology remaining comparable to the original for processing to evoke as little change as possible to the manufacturing process. For example, laminated doughs such as puff pastry require rolling of the dough and layering with fat (butter/shortening) to achieve lift during baking. These doughs are notoriously high in fat and efforts have been made to manufacture more healthful alternatives. Saturated fat content had at one time been replaced with trans fats, but this has been shown to be a less heathy option (Macierzanka et al., 2009; Simovic et al., 2009). Reduced fat formulations have been reported using W/O emulsions stabilized with fat crystals (Wickramarachchi et al., 2015). While dough rheological properties may be comparable during manufacture, moisture may increase due to coalescence of water droplets as the network melts during baking, which results in sub-optimal lift and sogginess (Wickramarachchi et al., 2015).

Lipsticks, which usually consist of an oil-continuous network of wax containing solid pigments, can incorporate a water phase to deliver hydrophilic compounds (flavouring, nutriceuticals, vitamins, etc) upon application (Beri et al., 2013; Le Révérend et al., 2011; Taylor, 2011). In emulsion-based lipstick, replacement of microcrystalline wax in cosmetic formulations may also benefit from strategic surfactant selection. For example, PGPR-stabilized droplets may promote adsorption of interfacial crystals which lead to a more reinforced structure in the presence of microcrystalline wax (Le Révérend et al., 2011). Replacing microcrystalline wax by a vegetable- 
based fat, the PGPR would have the opposite effect, preventing fat crystal adsorption and hindering network aggregates from interacting ultimately leading to a more viscous product.

Encapsulation is another field where fat-crystal W/O emulsions may play an increasingly important role. Studies have shown that the structure and organization of fat crystals around dispersed water droplets can be tuned to permit controlled release. Frasch-Melnik et al. investigated the effect interfacially crystallized tripalmitin had on the release of salt from W/O emulsions (Frasch-Melnik et al., 2010). They showed that presence of tripalmitin prevented the release of salt into a releasing medium with increasing tripalmitin improving encapsulation efficiency. In a similar study, Nadin et al. studied the release of $\mathrm{NaCl}$ from fat crystal network stabilized emulsions comparing Pickering and non-Pickering stabilization (Nadin et al., 2014). They found that interface composition was determinant in controlling release of encapsulated compounds from within a fat-crystal stabilized emulsion, with interfacial crystals completely covering the interface providing the most encapsulation potential.

Release of $\mathrm{FeSO}_{4}$ was carried out comparing PGPR-stabilized droplets in the presence or absence of rice bran wax (RBW) oleogels. RBW conferred greater stability to the otherwise unstable PGPR emulsions. The presence of RBW, especially at higher concentrations enhanced the encapsulation of $\mathrm{FeSO}_{4}$ through hindrance of droplet movement. RBW did not create Pickering shells around the droplets, but highlights that the presence of a crystal network alone may promote encapsulation of compounds simply through steric hindrance (Prichapan et al., 2017).

While there is evidence to support superior encapsulation potential from interfacial fat crystal stabilization under quiescent conditions, there is considerably less data on encapsulation and release properties of these emulsions under shear. Developing in vitro, bench-scale protocol for 
examining release under shear conditions is highly beneficial for many end-uses. Finally, replacement of fat or adding an aqueous phase is not necessarily turn-key from a manufacturing perspective. However, considering the interface as a contributing factor to structure may prove to have cost-saving and/or healthful benefits.

\subsection{Conclusion}

The rheology of fat crystal network-stabilized emulsions depends on many factors. In addition to the fat crystal network itself, the presence and activity of water droplets exert an influence on the rheological profile of the system and must be considered as a contributor in emulsion rheology, particularly in the presence of a fat crystal phase. As well, the aggregation behaviour of fat crystals, water droplets and mixtures of both is likely to tie into both droplet effective size and filler-filler interactions. Further affecting aggregation behaviour is the polydispersity of the dispersed droplet phase, which has been explored with solid particles in a fat crystal network, but not as much investigation has be done with liquid droplets (Afoakwa et al., 2008; Do et al., 2007). A mix of large and small droplets (or large and small fat crystal aggregates) may affect crystal/droplet packing, hence dramatically altering viscosity and rigidity (Sala et al., 2009; Van Aken et al., 2015). Clearly, inter-particle interactions must be better elucidated to create target products with different properties. 


\title{
Chapter 2 - Dispersed droplets as active fillers in fat-crystal network-
}

\section{stabilized water-in-oil emulsions}

Published in Food Research International (Rafanan and Rousseau, 2017)

\begin{abstract}
We show that the rigidity and microstructure of water-in-oil (W/O) emulsions depend on the ability of oil-soluble emulsifiers to enhance the crystallization of fats on the surface of dispersed aqueous droplets. In test emulsions consisting of hydrogenated soy oil (HSO) in liquid canola oil (CO) and a dispersed aqueous phase representing up to $20 \mathrm{wt} \%$ of the emulsion, use of glycerol monooleate (GMO) promoted oil-water interfacial crystallization whereas polyglycerol polyricinoleate (PGPR) resulted in HSO crystallization in the continuous phase only. By removing the confounding effects of droplet size and solid fat content, GMO-covered emulsion droplets were shown to behave as active fillers as they interacted with the surrounding fat crystal matrix and enhanced emulsion rigidity. By contrast, the PGPR-stabilized droplets only weakly associated with the matrix and did not significantly alter emulsion rheology, hence these were inactive fillers. This study shows that by simply changing emulsifier type, it is possible to alter the magnitude of the association between the dispersed droplets and surrounding fat crystals and, by extension, tailor the texture and rigidity of fat crystal-stabilized water-in-oil emulsions.
\end{abstract}




\subsection{Introduction}

From a materials science perspective, processed foods are composite materials consisting of a continuous matrix with multiple ingredients existing as solids, liquids, and/or gases. Ingredient properties such as inherent composition, physical state, volume fraction, and particle shape and size will alter the mechanical properties of a food (Ring and Stainsby, 1982). Beyond their mere presence, the extent of particle interactions between dispersed particulates and the surrounding matrix also impacts overall rheology and stability (Anton et al., 2001; Dickinson and Hong, 1995; Fröhlich et al., 2005).

The use of fillers is a well-established concept in the field of materials science, where embedded particulates dispersed within a continuous matrix exert a change on the rheological behaviour of a composite system. This dispersed phase can be considered an "active" or "inactive" filler where, as the former, it interacts strongly with the surrounding matrix and, as the latter, it demonstrates little to no interaction (Fröhlich et al., 2005; Genovese, 2012; McClements et al., 1993; Ring and Stainsby, 1982; van Vliet, 1988). Filler behaviour in food systems has been primarily studied in oil-in-water (O/W) emulsion gels (van Vliet, 1988). Notable properties dictating the rheological behaviour in these systems include oil droplet size and rigidity as well as how extensively the dispersed phase interacts with the surrounding matrix (Chen and Dickinson, 1995a; Dickinson and Hong, 1995; Genovese et al., 2010).

As a corollary to $\mathrm{O} / \mathrm{W}$ emulsion gels, fat crystal-stabilized water-in-oil (W/O) emulsions consist of a 3-D network of aggregated fat crystals held together by van der Waals forces (Maleky et al., 2012; Piska et al., 2006), whose rheology is affected by properties such as solid fat content, fat 
crystal polymorph and crystal aggregate size (Bell et al., 2007; de Man and Beers, 1988) as well as the presence of emulsifiers (Aronhime et al., 1988; Lawler and Dimick, 2002).

Water droplets embedded within a fat crystal network result in a composite material where the network comprises the matrix and the embedded water droplets the filler. It is known that the presence of a dispersed aqueous phase strongly impacts $\mathrm{W} / \mathrm{O}$ emulsion rheology, in particular at higher volume fractions where large increases in storage modulus $\left(\mathrm{G}^{\prime}\right)$ occur (Haj-shafiei et al., 2013; Johansson and Bergenståhl, 1995; Lopes da Silva and Coutinho, 2004; Macierzanka et al., 2009).

There remains a dearth of knowledge regarding how to tailor the properties of the oil-water interface to modify the rheology of fat crystal-stabilized W/O emulsions. In the absence of water droplets, emulsifiers affect fat crystallization by promoting or retarding crystal nucleation and growth depending on their concentration and fatty acid chain compatibility between the emulsifier and fat (Aronhime et al., 1988). In W/O emulsions, they will preferentially partition towards the oil-water interface, given their surface activity. Certain emulsifiers such as monoacylglycerols (MAGs) may promote triacylglycerol (TAG) adsorption to the interface better than others (Douaire et al., 2014; Ghosh and Rousseau, 2012; Haj-shafiei et al., 2013; Nadin et al., 2014), suggesting that this is a novel tool to alter emulsion rheology.

To our knowledge, the filler concept has yet to be applied to oil-continuous systems. Here, we extend it to fat crystal-stabilized W/O emulsion and investigate whether filler activity can be used to modulate emulsion rheology. The foundation of this study lies in the ability to control parameters such as creaminess, thickness and spreadability in foods and personal care products. These tunable qualities are essential for creating functional products such as low-fat spreads, where 
higher volumes of water can be incorporated to reduce saturated fats and caloric load or pharmaceutical preparations where minimal use of additives can be a marketable trait.

\subsection{Material and Methods}

\subsubsection{Emulsion formulation}

The W/O emulsions tested consisted of $5-20 \mathrm{wt} \%$ water with the remainder consisting of a 10 wt\% mixture of fat in oil and emulsifier. The constitutions of each emulsion are given in Table 2.1. The canola oil was purchased from a local supermarket (acid value $<0.2 \%$ ) and used without further purification. Prior to emulsion formation, hydrogenated soy oil (HSO) (Bunge, Bradley, IL, USA) was melted at $90{ }^{\circ} \mathrm{C}$ for 10 minutes to erase any crystal history. The HSO (acid value $0.02 \%$ ) consisted primarily of tristearin based on manufacturer specifications. Glycerol monooleate (GMO) (Monomuls90-O18, BASF Canada, Mississauga, ON, Canada) was present in all emulsions at a 0.1 GMO:water weight ratio while PGPR was added at a 0.01 PGPR:water weight ratio (PGPR) (Nealanders, Mississauga, ON, Canada). When optimizing formulations, as different ratios of emulsifier to water demonstrated the same behaviour, the lowest possible emulsifier concentration resulting in kinetically stable emulsions with similar droplet sizes was selected. As well, given that GMO exhibits complex phase behaviour at many water:surfactant ratios (Milak and Zimmer, 2015; Pitzalis et al., 2000), we chose a ratio that minimized such behaviour. The water:PGPR ratio was kept constant to surfactant concentration in the oil phase. 
Table 2.1 - Emulsion composition. Total emulsion mass was 200g.

\begin{tabular}{ccccc}
\hline & GMO & & & \\
\cline { 2 - 5 } Water $(\mathbf{w t})$ & Water & Surfactant & HSO & Canola oil \\
\hline $\mathbf{5}$ & 10 & 1 & 34.2 & 154.8 \\
$\mathbf{1 0}$ & 20 & 2 & 32.4 & 145.6 \\
$\mathbf{1 5}$ & 30 & 3 & 30.6 & 136.4 \\
$\mathbf{2 0}$ & 40 & 4 & 28.8 & 127.2 \\
\hline
\end{tabular}

\begin{tabular}{|c|c|c|c|c|}
\hline \multirow[b]{2}{*}{ Water (wt\%) } & \multicolumn{4}{|l|}{ PGPR } \\
\hline & Water & Surfactant & HSO & Canola oil \\
\hline 5 & 10 & 0.1 & 34.2 & 155.7 \\
\hline 10 & 20 & 0.2 & 32.4 & 147.4 \\
\hline 15 & 30 & 0.3 & 30.6 & 139.1 \\
\hline 20 & 40 & 0.4 & 28.8 & 130.8 \\
\hline
\end{tabular}

The aqueous phase consisted of $0.05 \mathrm{M} \mathrm{NaCl}$ and $1.46 \times 10^{-4} \mathrm{~mol} / \mathrm{L}$ Rhodamine. $\mathrm{NaCl}$ was added as distilled water alone promoted the formation of double emulsions rather than W/O emulsions during high pressure valve homogenization (Pradhan and Rousseau, 2012). Rhodamine allowed for better discernment of oil phase separation (oiling-off). It did not impact emulsification based on preliminary tests.

Emulsification with GMO was a three-step process. Pre-emulsions were prepared by dropwise addition of the aqueous phase to the molten fat, surfactant and oil blend by continuous magnetic stirring at $200 \mathrm{RPM}$ at $85-90{ }^{\circ} \mathrm{C}$ for 10 minutes. This was followed by emulsification with a rotor/stator mixer (PT 10/35 fitted with a general purpose, 2-knife aggregate, Kinematica, Inc., Bohemia, NY, USA) for 1 minute at $27000 \mathrm{rpm}$ and then high pressure valve homogenization (APV-1000, APV, Albertslund, Denmark) whose hopper was preheated to $85^{\circ} \mathrm{C}$. Homogenizer 
pressures were $48.3 \mathrm{MPa}\left(1^{\text {st }}\right.$ stage $)$ and $4.83 \mathrm{MPa}\left(2^{\text {nd }}\right.$ stage $)$. Once added to the hopper, the emulsions were cycled through the homogenizer for 5 minutes and immediately shear-cooled thereafter. PGPR-stabilized emulsions were emulsified by combining all phases together via rotorstator for 1 minute at $27,000 \mathrm{RPM}$ as no further homogenization was required to attain a comparable droplet size distribution to that of the GMO-stabilized emulsions.

All emulsions underwent the same shear cooling regimen at 260 RPM provided by a forwardcurved, 3-blade impeller powered by a motorized overhead stirrer (Stirrer: Model BDC3030, Blade: AC533, Caframo Lab Solutions Ltd., Georgian Bluffs, ON, Canada). The emulsion cooling chamber was maintained at $4{ }^{\circ} \mathrm{C}$ by partially submerging it in an ice bath. Emulsions were cooled from $75-85^{\circ} \mathrm{C}$ with shearing to a surface temperature of $13{ }^{\circ} \mathrm{C}$. Samples were then transferred to storage containers and analyzed for stability, rheology, microstructure, solid fat content and droplet size over 7 days.

\subsubsection{Solid fat content and fat crystal network characterization}

Emulsions were pipetted into NMR tubes to a height of $6 \mathrm{~cm}$ and subsequently stored at $4{ }^{\circ} \mathrm{C}$. Solid fat content (SFC) of the emulsions was determined at $4{ }^{\circ} \mathrm{C}$ using a temperature-controlled Bruker Minispec Mq20 (Bruker Canada, Milton, ON, Canada) with the application sfc_lfc v2.51.

Fat polymorphism was monitored by differential scanning calorimetry (DSC) and x-ray diffraction (XRD). DSC was conducted on a Q2000 DSC (TA instruments, New Castle DE, USA). Fresh 10$15 \mathrm{mg}$ aliquots of all emulsions and water-free controls were hermetically sealed within aluminum pans and heated from 5 to $90{ }^{\circ} \mathrm{C}$ at $5{ }^{\circ} \mathrm{C} /$ minute after equilibration at $5{ }^{\circ} \mathrm{C}$ for 1 minute. Peak 
melting temperatures and corresponding enthalpies were recorded. Wide-angle XRD was performed on a Hecus S3-MICROcaliX x-ray diffractometer (Hecus x-ray systems, Graz, Austria). Approximately $1 \mathrm{mg}$ samples were loaded into $1.0 \mathrm{~mm}$ quartz capillaries and x-ray signals were captured for 10 minutes, given the weak signal produced by the low solid fat proportion. The $\mathrm{x}$ ray source was provided by $50 \mathrm{kV}, 1 \mathrm{~mA} \mathrm{KCu} \mathrm{Ka}_{\alpha}$ anode. Samples in quartz capillaries were stored at $5{ }^{\circ} \mathrm{C}$ until the next sampling interval.

\subsubsection{Microscopy and droplet size determination}

All samples were characterized using a Carl Zeiss Axiovert 200M inverted light microscope at 630x magnification operated in brightfield and polarized light modes. Emulsion droplet size was determined through image analysis using ImageJ software (v1.51, Research Service Branch, National Institute of Health, Bethesda, MD). No fewer than 10 images per replicate were autothresholded to subtract the background and converted to binary images subjected to the "Analyze Droplets" algorithm using a scale of 8.8 pixels/micron. Each image per replicate was taken with the same light intensity and gain/offset settings and thus, had similar backgrounds. Autothresholding, rather than manual thresholding, allowed for the elimination of background noise while maintaining droplet outlines on each image before converting to a binary image. Since the same automatic algorithm (RenyiEntropy) was chosen for each image set, this eliminated variations in background subtraction and contrast adjustment between images. For a given aqueous phase wt $\%$, the highest number frequency for each trial was considered as the most representative droplet diameter. Droplet size determination was limited to image analysis as droplet diameters were $\sim 1 \mu \mathrm{m}$ and samples were non-dilutable. Hence, neither pfg-NMR nor light scattering was viable. Image analysis of brightfield micrographs was selected as it is non-destructive and reliable. 


\subsubsection{Small deformation rheology}

Oscillatory tests were performed at $5{ }^{\circ} \mathrm{C}$ on an Anton-Paar MCR301 rheometer (Anton Paar, St. Laurent, QC, Canada) equipped with a temperature-controlled Teflon stage using a parallel plate geometry (25 mm diameter, Teflon) with a gap of $0.5 \mathrm{~mm}$. Amplitude sweeps were conducted at $1.592 \mathrm{~Hz}$ from $0.01-100 \%$ strain. The linear viscoelastic region (LVR) was calculated using linear strain values deviating no more than $5 \%$ from baseline at low strain. Frequency sweeps were performed at the upper strain boundary of the LVR common to all samples. The frequency range was $0.01-10 \mathrm{~Hz}$.

\subsubsection{Distribution fitting and Statistics}

Analysis of variance (ANOVA) was performed with Tukey's HSD test when necessary using SigmaPlot 11 (Systat Software Inc., San Jose CA, USA). For all statistical tests, $\mathrm{n}=3$ and $\alpha=0.05$ was applied for all probability testing. Distribution fitting was processed using Matlab R2016a software (MathWorks, Natick, MA, USA)

\subsection{Results and Discussion}

\subsubsection{Visual appearance}

The GMO and PGPR-based emulsions studied for 7 days showed no phase separation at all water contents. Irrespective of water content, the PGPR-based emulsions exhibited a similarly thick, liquid consistency based on visual assessment. The GMO-based emulsions with 5 and 10 wt\% 
water produced a liquid similar in texture to the PGPR emulsions whereas at $15-20 \mathrm{wt} \%$ water, the emulsions were self-supporting soft solids. For comparison, the visual appearance of two 20 wt $\%$ W/O emulsions on day 0 demonstrates the flowable nature of the PGPR emulsion (Figure 2.1A) and semi-solid appearance of the GMO emulsion (Figure 1B). Overall, the incorporation of water in the GMO-based emulsions produced significant textural differences observable by the naked eye, but a similar increase in rigidity was not observed in PGPR-based emulsions.

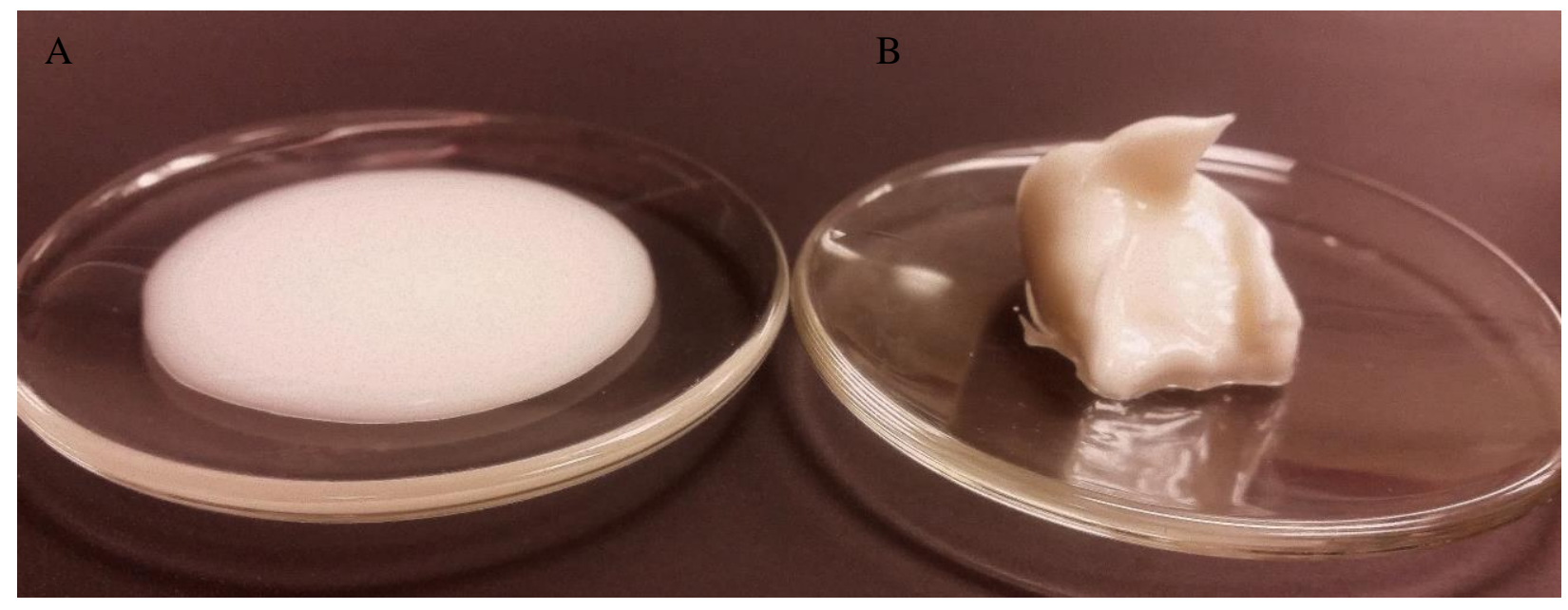

Figure 2.1 - Appearance of freshly-prepared $20 \mathrm{wt} \% \mathrm{~W} / \mathrm{O}$ emulsions prepared with (A) PGPR and (B) GMO. SFC, water content and droplet size were matched in both emulsions.

\subsubsection{Microstructure}

The initial droplet sizes in the GMO and PGPR emulsions at all water contents were comparable, with average droplet diameters of $1-3 \mu \mathrm{m}$ and no droplets $>10 \mu \mathrm{m}$ in diameter (Appendix table 2.1). Figure 2 compares the brightfield and polarized light images of $20 \mathrm{wt} \% \mathrm{~W} / \mathrm{O}$ emulsions prepared with GMO (Figures 2.2A and B) and PGPR (Figure 2.2C and D). The GMO-based 
emulsions consisted of discrete droplets existing as aggregates (Figure 2.2A circle) or within fat crystalline shells (Figure 2.2A arrow), with very few droplets freely existing within the continuous phase. This behaviour was particularly apparent in emulsions containing 15 and $20 \mathrm{wt} \%$ water. At 5-10 wt $\%$ water, droplets were more evenly spaced, though still adsorbed to the surface of fat crystal aggregates. The crystalline oil-water interface was apparent in all GMO-stabilized emulsions regardless of water content. Evidence of interfacial crystallization was also confirmed given that many of the larger droplets were generally spherical, but their surfaces were not smooth, appearing wrinkled, which was consistent with previous studies (Figure 2.2B inset) (Beri et al., 2013; Macierzanka et al., 2009). 

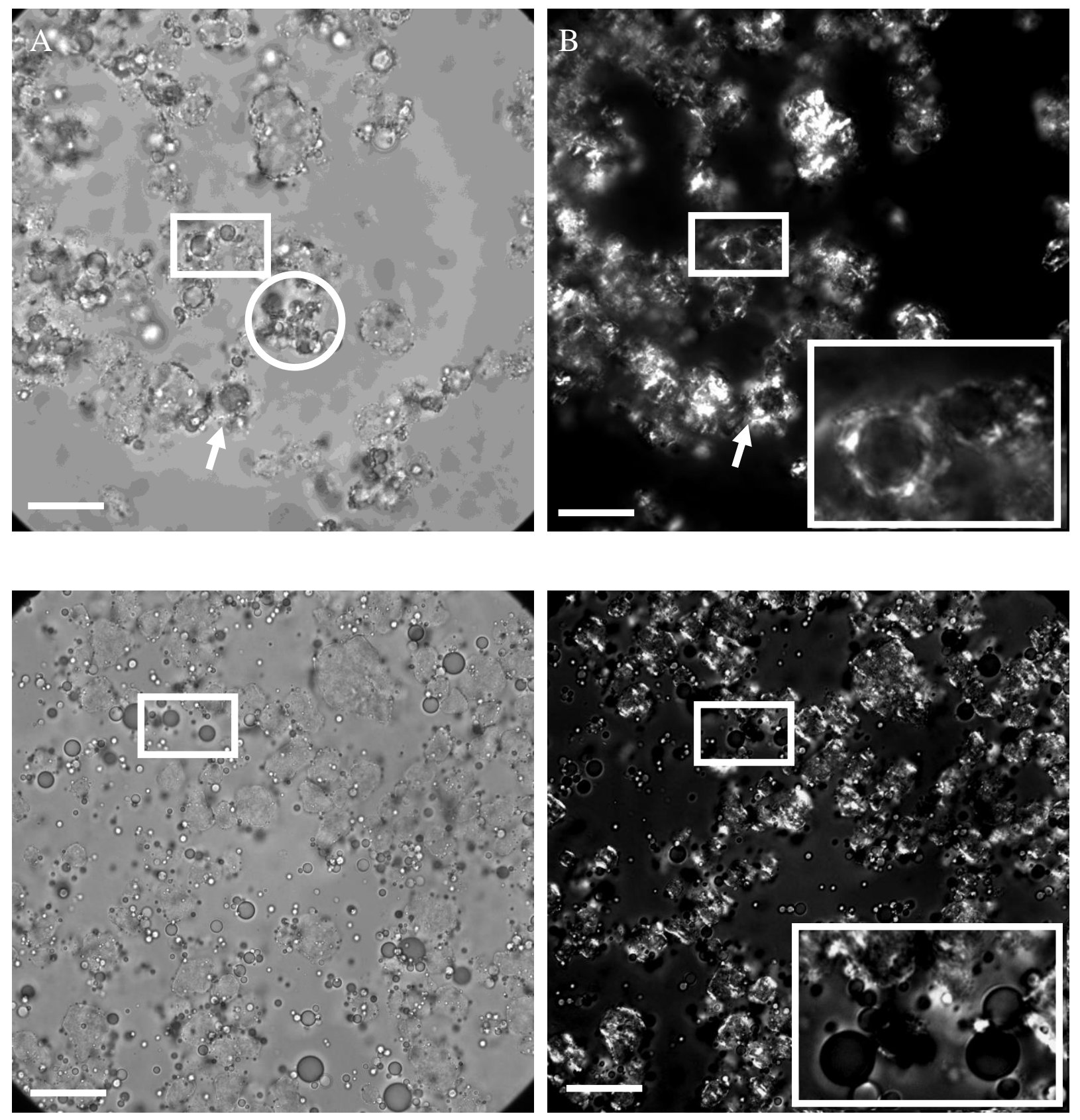

Figure 2.2 - Micrographs of freshly-prepared $20 \mathrm{wt} \% \mathrm{~W} / \mathrm{O}$ emulsions containing either GMO or PGPR. GMO-based emulsion: (A) brightfield and (B) polarized light; PGPR-based emulsion: (C) brightfield and (D) polarized light. Insets in B and D are zoomed-in views of boxed areas. Boxed areas highlight the droplet interface, indicating the crystalline interface of GMO-stabilized emulsions and the lack thereof in the PGPR-stabilized emulsion. Circled area in A shows a droplet aggregate consisting of small, clustered droplets and fat crystals. The arrow in A shows a droplet encased within a fat crystal shell. The hazy appearance of the PGPR interface is due to wall effect caused by the liquid interface. Scale bar is $20 \mu \mathrm{m}$. 
A different microstructure emerged with PGPR-based emulsions, where most droplets were freely moving throughout the continuous phase (Figures 2.2C and 2.2D). At all water concentrations, there was no evidence of flocculation and the overall number of freely-moving droplets increased with increasing water content. The morphology of the PGPR emulsions was dominated by a fat crystal network consisting of aggregated smoothened spherulites and non-interacting, individual aqueous droplets interspersed throughout (Figure 2.2D inset). Though these images depict the same field of view, brightfield and polarized light images taken seconds apart clearly showed the mobility of the fat crystal aggregates and droplets. No such mobility was apparent in the GMObased emulsions.

From a mechanistic perspective, during homogenization, the GMO rapidly diffused and adsorbed onto the oil-water interface where it self-assembled resulting in a brush layer, with its polar groups (hydroxyl and carbonyl groups) residing in the aqueous side of the interface and the acyl chains exposed to the oil phase. In the presence of a molten high-melting fat such as HSO in the continuous phase, association between the GMO and HSO fatty acid chains occurred through van der Waals interactions, which was promoted by their similar acyl chain lengths match (i.e., oleic acid in the GMO and stearic acid in the HSO). During cooling, below the HSO crystallization temperature, the associated acyl chains in the emulsifier and fat co-solidified via interfacial HSO TAG heterogeneous nucleation (Bayés-García et al., 2015). Presumably, templated crystal growth of highly-aligned TAGs at the oil-water interface proceeded as the crystals thickened, spread and englobed entire droplets with a solid fat crystal shell. Though not studied in emulsions, the Dewettinck group also noted the templating effects of unsaturated MAGs, which they found was less apparent than their saturated counterparts (Foubert et al., 2004). 
Such behaviour was in contrast to the PGPR-based emulsions where no evidence of an interfacial fat layer was apparent on the droplets. Given its larger size and polymeric nature, PGPR did not form an ordered monolayer upon adsorption to the oil-water interface capable of effectively interacting with the surrounding HSP TAGs. As a result, it was not capable of promoting interfacial fat crystallization (Ghosh and Rousseau, 2009; Rousseau et al., 2009; Wassell et al., 2012). Rather than being adsorbed to the oil-water interface, the HSO was present as distinct, smoothened spherulites in the continuous fat phase with no associated droplets. As discussed below, these distinct differences in droplet and fat crystal spatial distribution were largely responsible for significant differences in rheological properties.

\subsubsection{Fat crystal properties}

The SFC, polymorphism and thermal behaviour of the emulsions were investigated. The SFC was statistically similar in both GMO and PGPR emulsions at all water contents ( $\mathrm{p}>0.05)$, though a small decrease occurred between days 0 and $7(\mathrm{p}>0.05)$ (Table 2.2). X-ray diffraction revealed that fat crystals in both freshly-prepared GMO and PGPR-based emulsions were in the $\beta$ polymorph which persisted over 7 days, with wide-angle d-spacings showing a dominant peak at 4.6 $\AA$, with lesser peaks at $3.7 \AA$ and $3.9 \AA$ for all emulsions (Da Silva et al., 2009; Lutton, 1945) $(\mathrm{p}>0.05)$. In the absence of emulsifier and water, HSO crystals also existed in the $\beta$ polymorph, showing that neither emulsifier promoted a change in TAG subcell arrangement. Though the incorporation of saturated MAGs into growing TAG lattices affects fat crystal polymorphism (Fredrick et al., 2008), neither GMO or PGPR show strong effects (Garti et al., 1998; Niiya et al., 1973a, 1973b, 1973c). 
Table 2.2: Solid fat content of HSO in the W/O emulsions as a function of time, water content and emulsifier type. Different lower-case superscript letters denote within-column statistical differences in SFC as a function of water content. Different upper-case superscript letters denote between-column statistical differences in SFC as a function of time for each emulsifier. No differences in $(n=3)$.

\begin{tabular}{ccccc}
\hline & \multicolumn{2}{c}{ GMO } & \multicolumn{2}{c}{ PGPR } \\
Water content $(\mathbf{w t} \%)$ & Day 0 & Day 7 & Day 0 & Day 7 \\
\hline $\mathbf{0}$ & $10.6 \pm 0.2^{\mathrm{aA}}$ & $10.6 \pm 0.3^{\mathrm{aA}}$ & $10.7 \pm 0.3^{\mathrm{aA}}$ & $10.6 \pm 0.4^{\mathrm{aA}}$ \\
$\mathbf{5}$ & $10.6 \pm 0.2^{\mathrm{aA}}$ & $10.2 \pm 0.2^{\mathrm{aA}}$ & $10.7 \pm 0.2^{\mathrm{aA}}$ & $10.2 \pm 0.2^{\mathrm{aB}}$ \\
$\mathbf{1 0}$ & $10.0 \pm 0.1^{\mathrm{bA}}$ & $9.2 \pm 0.4^{\mathrm{bB}}$ & $9.9 \pm 0.1^{\mathrm{bA}}$ & $9.2 \pm 0.4^{\mathrm{bB}}$ \\
$\mathbf{1 5}$ & $9.2 \pm 0.2^{\mathrm{cA}}$ & $9.0 \pm 0.2^{\mathrm{bA}}$ & $9.0 \pm 0.1^{\mathrm{cA}}$ & $9.0 \pm 0.2^{\mathrm{bA}}$ \\
$\mathbf{2 0}$ & $9.0 \pm 0.5^{\mathrm{cA}}$ & $8.6 \pm 0.3^{\mathrm{bA}}$ & $9.0 \pm 0.4^{\mathrm{cA}}$ & $8.6 \pm 0.3^{\mathrm{cA}}$ \\
\hline
\end{tabular}

DSC measurements showed no significant difference in fat melting point between days 0 and 7 (Appendix table2.2). However, there was a small, but significant difference between the melting peaks of the GMO and PGPR-based emulsions: $54^{\circ} \mathrm{C}$ vs. $55^{\circ} \mathrm{C}$, respectively $(\mathrm{p}<0.05)$. On a fat mass basis, the melting enthalpy $(\Delta \mathrm{H})$ of the HSO in oil was significantly higher at $\sim 167 \mathrm{~J} / \mathrm{g} \mathrm{HSO}$ than in all emulsions, as well as when it was blended with both oil and emulsifier (Table 2.3) (p < 0.05). This suggested that the presence of either emulsifier or water reduced the $\Delta \mathrm{H}$ of HSO. Though the reported enthalpies coincided with those consistent with $\beta^{\prime}$-tristearin, XRD did indicate the $\beta$-polymorph. The decrease in $\Delta \mathrm{H}$ (and SFCs) was speculatively attributed to a dilution effect of the fat in oil or perhaps interactions with the interface. 
Table 2.3: Melting enthalpy $(\mathrm{J} / \mathrm{g})$ of $\mathrm{HSO}$ in the $\mathrm{W} / \mathrm{O}$ emulsions as a function of time, water content and emulsifier type. Enthalpy values are reported based on mass HSO per emulsion. Different lower-case superscript letters denote within-column statistical differences in SFC as a function of water content. Different upper-case superscript letters denote between-column statistical differences in SFC as a function of time for each emulsifier $(n=3)$.

\begin{tabular}{ccccc}
\hline \multirow{2}{*}{ Water content (wt\%) } & \multicolumn{2}{c}{ GMO } & \multicolumn{2}{c}{ PGPR } \\
& Day 0 & Day 7 & Day 0 & Day 7 \\
\hline $\mathbf{0}$ & $151.1 \pm 5.8^{\mathrm{aA}}$ & $162.7 \pm 4.2^{\mathrm{aB}}$ & $153.3 \pm 1.5^{\mathrm{aA}}$ & $158.4 \pm 3.3^{\mathrm{aB}}$ \\
$\mathbf{5}$ & $154.0 \pm 10.5^{\mathrm{abA}}$ & $143.8 \pm 0.7^{\mathrm{bA}}$ & $144.8 \pm 5.9^{\mathrm{bA}}$ & $147.7 \pm 4.0^{\mathrm{bB}}$ \\
$\mathbf{1 0}$ & $142.7 \pm 2.2^{\mathrm{bcA}}$ & $140.2 \pm 2.6^{\mathrm{bA}}$ & $138.5 \pm 3.9^{\mathrm{bA}}$ & $154.8 \pm 2.3^{\mathrm{aB}}$ \\
$\mathbf{1 5}$ & $145 \pm 11.8^{\mathrm{abcA}}$ & $147.6 \pm 8.6^{\mathrm{bA}}$ & $140.4 \pm 2 .^{\mathrm{bA}}$ & $154.8 \pm 1.9^{\mathrm{aB}}$ \\
$\mathbf{2 0}$ & $136.5 \pm 5.7^{\mathrm{bcA}}$ & $139.8 \pm 8.3^{\mathrm{bA}}$ & $123.5 \pm 3.5^{\mathrm{cA}}$ & $138.1 \pm 1.6^{\mathrm{cB}}$ \\
\hline
\end{tabular}

\subsubsection{Emulsion and bulk fat rheology}

Oscillatory rheology performed on day 0 and 7 samples revealed clear differences in storage $\left(\mathrm{G}^{\prime}\right)$ and loss $\left(G^{\prime \prime}\right)$ moduli. GMO had a greater influence on $G^{\prime}$ than $G^{\prime \prime}$ for both amplitude- and timedependent emulsion properties than PGPR, which was most evident at higher water contents.

Amplitude sweeps of the emulsions gave LVRs at 0.01-0.03\% strain for all emulsions, regardless of water content or emulsifier type. The GMO and PGPR-containing controls (i.e., fat, oil and emulsifier only) were statistically similar $(p>0.05)$ in terms of $\mathrm{G}^{\prime}$ and $\mathrm{G}^{\prime \prime}$ moduli within the LVR.

For the GMO-based emulsions, the $\mathrm{G}^{\prime}$ values were consistently higher than $\mathrm{G}^{\prime \prime}$ on both days 0 and 7 within the LVR (Figure 2.3). On day 0, the emulsions with 5 and $10 \mathrm{wt} \%$ water did not differ in respect to $G^{\prime}$ and $G^{\prime \prime}$ from the control $(p>0.05)$ and demonstrated a consistency similar to liquid 
shampoo. Significant differences were observed with 15 and $20 \mathrm{wt} \%$ water, with the latter exhibiting the highest $\mathrm{G}^{\prime}$ value $(\sim 1500 \mathrm{~Pa})$ of all formulations $(\mathrm{p}<0.05)$ on day 0 .

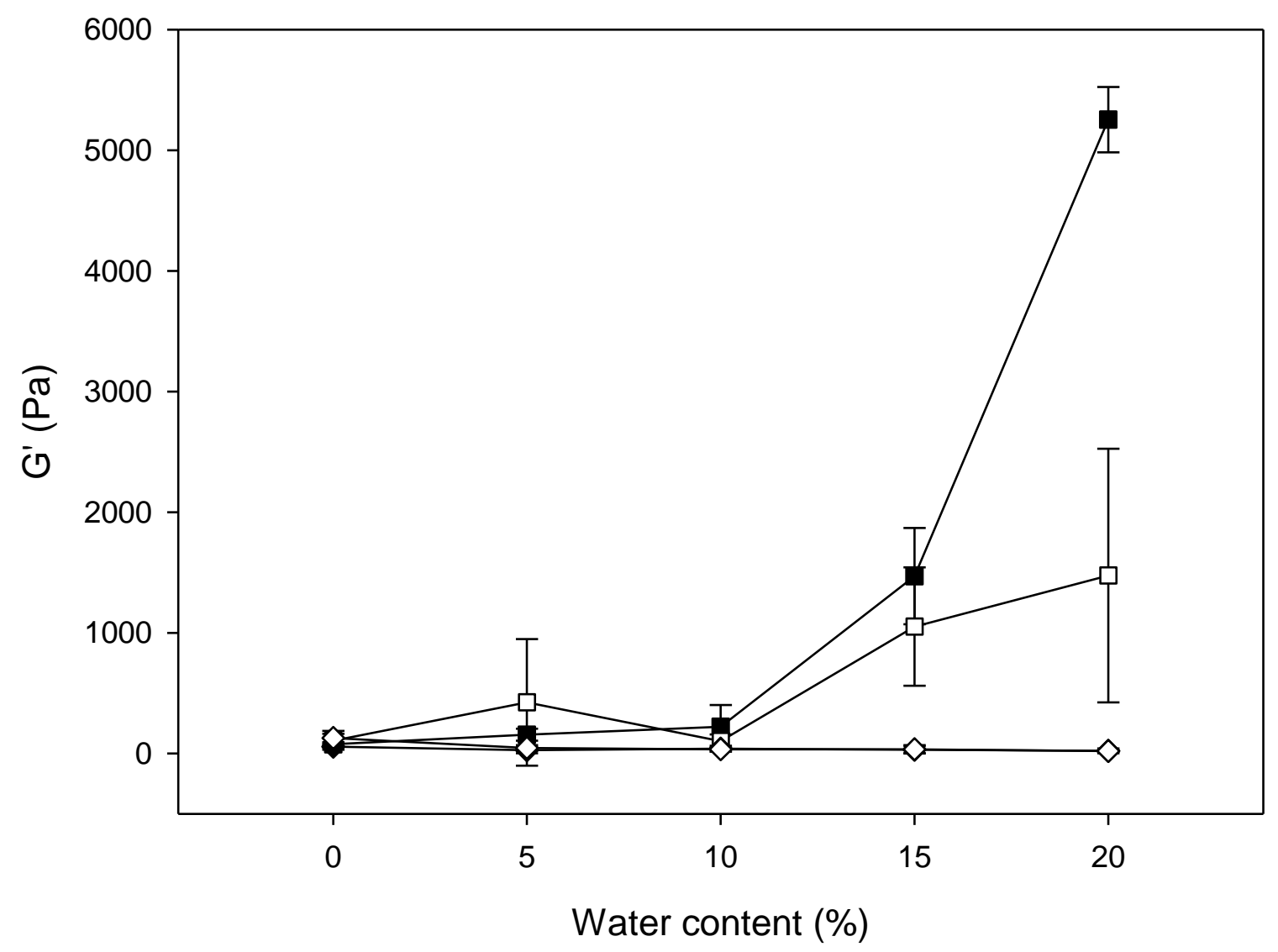

Figure 2.3 - The amplitude-dependent storage modulus $\left(\mathrm{G}^{\prime}\right)$ of GMO and PGPR-based emulsions at days 0 and 7. GMO day 0 (- $\square-)$ and day 7 (-ם-); PGPR day 0 (- - -) and day 7 (- $>-)$. Day 0 and 7 PGPR emulsion data is overlapping. Error bars show standard deviation $(n=3)$.

Ageing significantly affected the $\mathrm{G}^{\prime}$ of the GMO-based emulsions. Though there was no statistical difference in $\mathrm{G}^{\prime}$ or $\mathrm{G}^{\prime \prime}$ during storage $(\mathrm{p}>0.05)$ at low water content, with 15 and $20 \mathrm{wt} \%$ water, $\mathrm{G}^{\prime}$ increased 1.5 times to $\sim 1500 \mathrm{~Pa}$ and 3.5 times to $\sim 5200 \mathrm{~Pa}$ from days 0 to 7 , respectively. These emulsions with the higher aqueous phase content exhibited a soft solid consistency similar to 
mayonnaise. Beyond the expected increase in $\mathrm{G}^{\prime}$ arising from the presence of $15-20 \mathrm{wt} \%$ water in the form of dispersed droplets, this consistency arose from sintering between droplets and network aggregates given the similar SFCs (Garbolino and Bartoccini, 2005; Johansson and Bergenståhl, 1995).

The PGPR-based emulsions did not exhibit changes in $\mathrm{G}^{\prime}$ or $\mathrm{G}^{\prime \prime}$ with composition and storage (p>0.05) and displayed a thick liquid-like character irrespective of water content. In keeping with the visual assessment in Figure 1, G' and $\mathrm{G}^{\prime \prime}$ overlapped within the LVR and did not exceed 100 Pa. Therefore, with similar droplet sizes, water contents and SFCs, emulsions stabilized by GMO resulted in a self-supporting soft solid whereas PGPR yielded viscous liquids.

Frequency sweeps from 0.01 to $10 \mathrm{~Hz}$ performed at $0.02 \%$ strain are presented as $\tan \delta\left(=\mathrm{G}^{\prime \prime} / \mathrm{G}^{\prime}\right)$ in Figure $2.4\left(\mathrm{G}^{\prime}\right.$ and $\mathrm{G}^{\prime \prime}$ shown in Appendix figure 2.1). A $\tan \delta$ value of 1 indicates a threshold point above which a material is predominantly viscous whereas values $<1$ represent dominant elastic behaviour. The $\tan \delta$ value was useful in explaining the changes that occurred during ageing in the present emulsions, in particular the role of continued fat post-crystallization (i.e. sintering) with time and the possible role of droplet-crystal floc formation on rheology. 

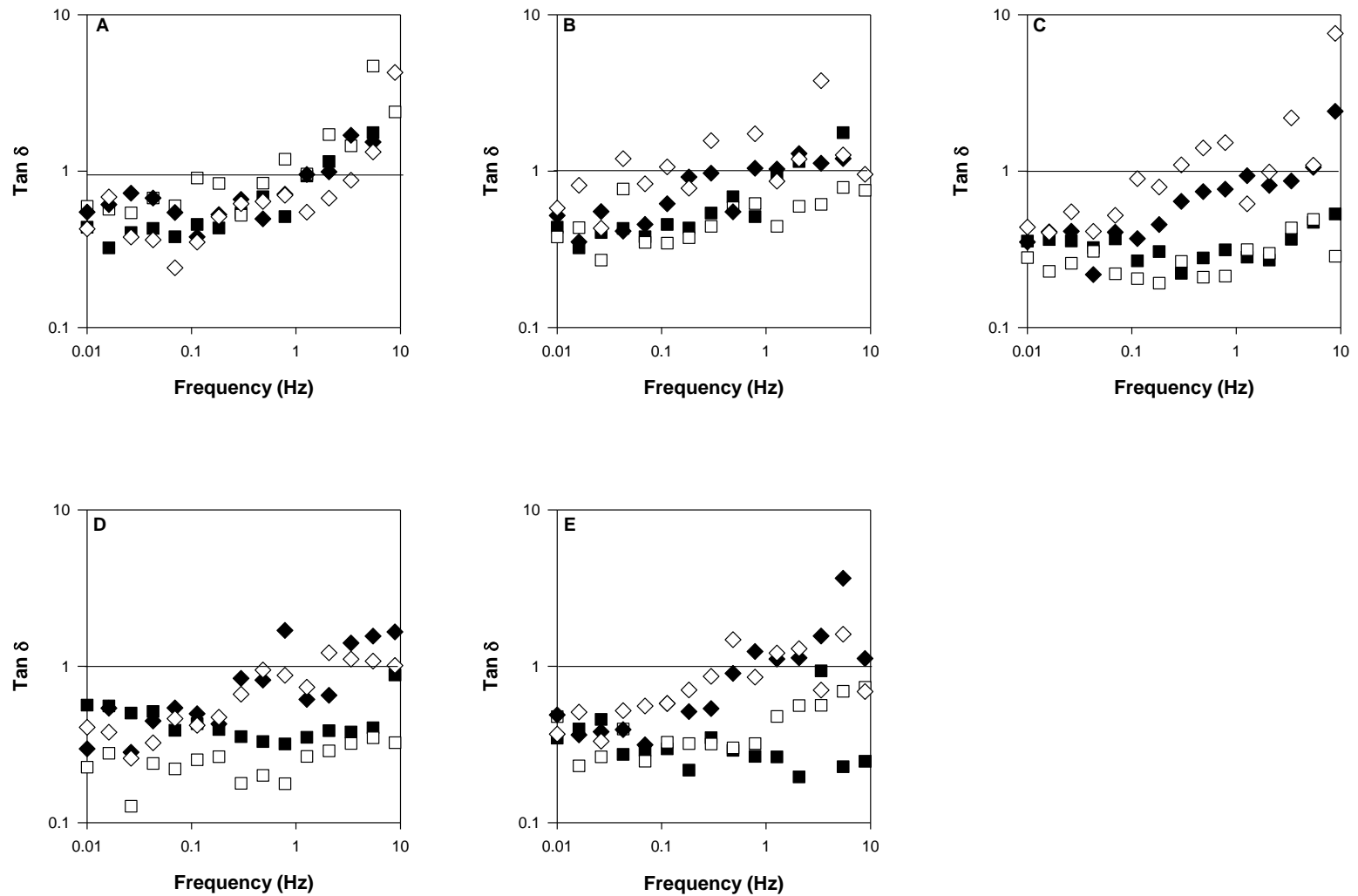

Figure 2.4 - Frequency sweeps from 0.01 to $10 \mathrm{~Hz}$ of GMO and PGPR-based emulsions at days 0 and 7. Viscoelastic character given as $\tan \delta=\mathrm{G}^{\prime \prime} / \mathrm{G}^{\prime}$. Water content: A - $0 \mathrm{wt} \%$ (bulk); B - $5 \mathrm{wt} \%$; C - $10 \mathrm{wt} \%$; D - $15 \mathrm{wt} \%$; E - $20 \mathrm{wt} \%$. GMO day 0 (- $\square-)$ and day 7 (-ם-); PGPR day 0 (- $\diamond-)$ and day 7 (-). Horizontal line at $\tan \delta=1$ shows threshold between predominantly elastic (below) and viscous behaviour (above). Values above $10 \mathrm{~Hz}$ in $4 \mathrm{~A}$ removed due to slippage. Error bars removed for clarity $(n=3)$.

Overall, all PGPR-based emulsions demonstrated frequency dependence whereas the GMO-based emulsions shower no frequency dependence, but only at higher water content. On day 0 , the bulk fat and $5 \mathrm{wt} \%$ water emulsions displayed frequency-dependent weakening above $\sim 1 \mathrm{~Hz}$, with similar behaviour evident for both emulsifiers (Figures 2.4A and 4B), suggesting a shift from an elastic to a viscous response at higher frequencies. At $\geq 10 \mathrm{wt} \%$ water, emulsifier type significantly impacted $\tan \delta$ frequency dependence. The GMO-based emulsions showed $\tan \delta$ values $<1$ at all 
frequencies, indicative of a greater solid-like character (Figures 2.4C-E). By contrast, the PGPRbased emulsions still displayed extensive frequency-dependent deformation $(\tan \delta>1)$ (Figures 2.4C-E).

Storage for one week substantially altered the $\tan \delta$ evolution of the GMO but less so the PGPRbased emulsions. Contrary to the emulsions, the bulk fats showed little change in $\tan \delta$ evolution over time (Figure 2.4A), indirectly demonstrating that presence of a dispersed aqueous phase contributed to strengthening of all emulsions via an increase in $\mathrm{G}^{\prime}$ or reduction in $\tan \delta$. All PGPRbased emulsions showed lower $\tan \delta$ values at day 7 vs day 0 , though a transition to viscous behaviour occurred between 0.1 and $1 \mathrm{~Hz}$ irrespective of aqueous phase wt $\%$ (Figure 2.4B-E). The GMO emulsion with $5 \mathrm{wt} \%$ water also demonstrated a transition from elastic to viscous flow. At $\geq 10 \mathrm{wt} \%$ water, only elastic behaviour was evident based on the $\tan \delta$ values below 1 irrespective of frequency (Figure 2.4B-E)

The combination of higher $\mathrm{G}^{\prime}$ and lower $\mathrm{G}^{\prime \prime}$ in the GMO-based emulsions at higher water content reflected a more rigid, stiff material whose $\tan \delta$ value decreased with time. The gradually lower $\tan \delta$ values indicated a material whose recovery characteristics were improved as the structural elements within (whether droplets, fat crystals or their combination) either resisted deformation more effectively or regained their original structure more rapidly following the application of stress. Reinforcement of the matrix was concentration-dependent and relied on the interaction of the droplets with the surrounding fat crystals to form a droplet-crystal flocculated network. Considering that flocs were not observed at lower water concentrations (i.e.,, 5 and $10 \mathrm{wt} \%$ water GMO emulsions), such flocculation was pivotal in enhancing the rigidity of these W/O emulsions (Macierzanka et al., 2009). 
Frequency sweep $\mathrm{G}^{*}$ data was fitted to a power law distribution where $\mathrm{G}^{*}=a \omega^{b}$ (Table 2.4) (Subramanian et al., 2006). All fits displayed a high degree of correlation $\left(\mathrm{R}^{2}>0.93\right)$. In the GMOstabilized emulsions, the increase in the value of $a$ indicated greater resistance to time-dependent deformation with increasing water content, particularly at lower frequencies. There was little evolution in parameter $a$ for all PGPR-stabilized emulsions over time, suggesting the absence of changes in viscoelastic character. Beri et al noted similar behaviour in wax systems where only certain additives incorporated into wax crystal networks increased $\mathrm{G}^{\prime}$ (Beri et al., 2013). Here, GMO-stabilized droplets interacted with the surrounding fat crystals in the network whereas PGPR-stabilized droplets did not promote such links. Parameter $b$ was similar in all emulsions irrespective of surfactant, age or aqueous phase $\mathrm{wt} \%(\mathrm{p}>0.05)$, indicating similar shear-dependent softening rate in all samples. Negative values for the $b$ variable are not expected, indicating that this while this particular model exhibits fairly good correlation with the data and does describe general trends in the $a$ variable, it does not adequately describe structural deformation under shear. Fitting $\mathrm{G}^{\prime}$ and $\mathrm{G}^{\prime \prime}$ to the above model had similar trends in $a$ and $b$. 
Table 2.4: Power law model fit of frequency sweep $\mathrm{G}^{*}$ values for fat crystal network-stabilized emulsions containing $0-20 \mathrm{wt} \%$ water over time, where $\mathrm{G}^{*}=\mathrm{a} \omega^{\mathrm{b}}$. All fits displayed a high degree of correlation $\left(\mathrm{R}^{2}>0.93\right)$.

\begin{tabular}{cccccc}
\hline & \multicolumn{2}{c}{ Day 0 } & \multicolumn{2}{c}{ Day 7 } \\
\hline WMO & Water $(\mathbf{w t} \%)$ & $\mathrm{a}(\mathrm{Pa})$ & $\mathrm{b}$ & $\mathrm{a}(\mathrm{Pa})$ & $\mathrm{b}$ \\
& $\mathbf{0}$ & 19.2 & -1.0 & 19.2 & -1.0 \\
& $\mathbf{5}$ & 224.8 & -1.0 & 224.8 & -1.0 \\
& $\mathbf{1 0}$ & 332.7 & -1.1 & 332.7 & -1.1 \\
& $\mathbf{1 5}$ & 645.6 & -0.9 & 645.6 & -0.9 \\
\hline PGPR & $\mathbf{2 0}$ & 711.3 & -0.9 & 691.3 & -0.9 \\
& $\mathbf{0}$ & 67.6 & -0.8 & 18.0 & -0.9 \\
& $\mathbf{5}$ & 10.2 & -0.9 & 18.0 & -1.1 \\
& $\mathbf{1 0}$ & 6.7 & -1.2 & 27.6 & -0.8 \\
& $\mathbf{1 5}$ & 21.4 & -0.9 & 9.3 & -1.2 \\
& $\mathbf{2 0}$ & 8.6 & -1.0 & 15.4 & -0.8 \\
\hline
\end{tabular}

Based on their distinct contributions to emulsion rheology, the GMO- and PGPR-covered dispersed droplets were classified as either active or inactive fillers. Though emulsions with a higher dispersed phase volume fraction normally display greater thickening due to an increase in droplet-droplet interactions (Koenig et al., 2002), this was not observed with the PGPR-stabilized emulsions as $\mathrm{G}^{\prime}$ remained constant at all water contents, and was similar to the bulk fat $(\mathrm{p}>0.05)$. Such inability to reinforce the fat crystal network categorized the PGPR-stabilized droplets as an inactive filler. Conversely, the GMO-stabilized droplets, in particular at high volume fractions (i.e., 15 and $20 \mathrm{wt} \%$ ), were active fillers as they significantly increased $\mathrm{G}^{\prime}$. Such enhancement was absent at lower water contents (5 and $10 \mathrm{wt} \%$ ), suggesting a threshold content for this behaviour.

There was clustering of droplets into flocs in the GMO-stabilized emulsions with 15 and 20 wt $\%$ water (Figure 2.2A and B). As previously shown in $\mathrm{O} / \mathrm{W}$ emulsion gels, droplet clusters can assist 
in gel formation and the redistribution of shear stresses by behaving as larger particles (Sala et al., 2009). A compounding factor in the present study was the added rigidity of the fat crystal spheroids englobing the droplets, which further increased the effective volume fraction of the dispersed aqueous phase, and by extension, emulsion $\mathrm{G}^{\prime}$. By comparison, particles with limited connectivity with the surrounding matrix would not redistribute shear forces and as such, no reinforcement should be observed (Van Aken et al., 2015).

The Norton group noted that the hardness of wax crystal-stabilized W/O emulsions generally decreased with increasing dispersed aqueous phase content. They proposed that wax crystals moving to the water droplet surface and away from the bulk weakened the network structure as the lower number of wax crystals present led to fewer crystal-crystal junctions (Le Révérend et al., 2011). Similarly, the present study showed that crystal-crystal contact was determinant in rheological reinforcement. Though the dispersed water droplets behaved as 'structural defects' in the network by reducing the number of contact points between neighbouring fat crystals, droplets with adsorbed fat crystals more effectively interacted either with the surrounding network and/or adjacent droplets to reinforce the material.

The Le Révérend et al. study also suggested that PGPR, which is derived from castor oil and which shared 'molecular similarities' (e.g. acyl chain lengths and polarity) with the surrounding solvent oil in the continuous phase, promoted solid wax adsorption onto the interface (Le Révérend et al., 2011). Yet, the present study and those of others, which used divergent solvent oils and fats, showed the very opposite (Ghosh and Rousseau, 2012). Irrespective of whether solids adsorption to the interface is mediated by favourable molecular similarity (Ghosh and Rousseau, 2012) or adsorption through favourable particle size/shape (Madivala et al., 2009), this showed that the 
droplet surface acted as a functional component in the emulsions, either by sequestering components from the matrix or by contributing to structural integrity.

Finally, Middendorf et al. reported that PGPR appeared to create emulsifier-rich regions within a cocoa butter-sugar matrix (Middendorf et al., 2015). 'PGPR pillows', as the authors stated, acted as spacers between adjacent sugar particles and promoted viscous flow of the cocoa butter-sugar mixtures. In the current study, PGPR-stabilized droplets similarly acted as spacers between adjacent fat crystal aggregates, mitigating fat crystal interactions and promoting flow. By contrast, GMO at the oil-water interface anchored adjoining fat crystals, resulting in a more mechanically rigid interface compared to PGPR.

\subsection{Conclusion}

The rheological properties of fat crystal-stabilized W/O emulsions can be tuned by simply altering the emulsifier present at the oil-water interface, similarly to how active and inactive fillers alter rheological behaviour in composite gels. This effect depends on the number of water droplets dispersed within the network which, in turn, governs the viscoelastic behaviour of these dispersions. Interfacial crystallization promoted by GMO increased $\mathrm{G}^{\prime}$, particularly at $\geq 15 \mathrm{wt} \%$ dispersed water whereas PGPR was passive and did not promote interfacial crystallization or reinforce the network, as evidenced by the lack of change in $\mathrm{G}^{\prime}$ with increasing water content.

To our knowledge, categorizing droplets in W/O emulsions as active or inactive fillers is a new concept, where we can no longer think of emulsions as simply one phase dispersed within another, but as a highly complex collection of modifiable, interactive surfaces. Droplet deformation must 
be taken into account, which is not a consideration with solid fillers. By structuring the interface, it is possible to more predictably tailor rigidity, deformation and storage stability. This work can be applied to numerous industries, such as food, pharma and cosmetics, where desired texture modification can be attained with modest changes in formulation. 


\title{
Chapter 3 - Dispersed droplets as tunable fillers in water-in-oil
}

\section{emulsions stabilized with fat crystals}

Published in Journal of Food Engineering (Rafanan and Rousseau, 2019a)

\begin{abstract}
The role of water droplets as rheology modifiers in fat crystal-stabilized emulsions is not widely studied, but relevant to many fields and industries such as foods and cosmetics. The degree to which water droplets interact with the surrounding fat crystal network can impart a significant effect on rheology. The objective of this study was to investigate how the rheology of water-in-oil (W/O) emulsions stabilized by a surrounding fat crystal network is impacted by the ability of emulsifiers that promote interfacial fat crystallization and whether droplet size is a contributing factor. Three emulsifiers imparting different interfacial characteristics were chosen: glycerol monooleate (GMO) and glycerol monostearate (GMS) both promoted interfacial crystallization in the presence of hydrogenated soy oil (HSO) whereas polyglycerol polyricinoleate (PGPR) did not. Different homogenization methods were used to create emulsions with two distinct average droplet sizes with narrow size distributions for each emulsifier. Amplitude sweeps showed that GMS emulsions, independently of droplet size, displayed the highest elastic modulus $\left(\mathrm{G}^{\prime}\right)$ values

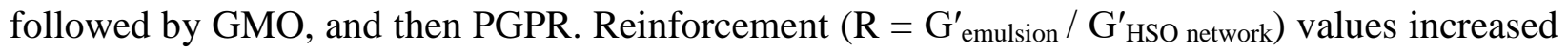
with each emulsifier over time, but its extent was dependent on emulsifier-mediated dropletdroplet interactions. In both GMO and GMS-stabilized emulsions, smaller droplets enhanced reinforcement whereas the opposite was observed in PGPR-stabilized emulsions. This study has shown that, through the use of different emulsifiers, the droplet interface should be considered a
\end{abstract}


functional component that can used to tailor the consistency of fat crystal-stabilized W/O emulsions. 


\subsection{Introduction}

Fat crystal-stabilized emulsions are complex mixtures present in consumer products including spreads such as butter and margarine as well as cosmetic preparations as cold cream and lipstick. They comprise a dispersed aqueous phase sterically stabilized by a 3D matrix of crystallized triacylglycerols (TAGs) (de Man and Beers, 1988; Hodge and Rousseau, 2005). Droplets in such emulsions can be stabilized by various means, namely small-molecule emulsifiers or solid species present at the droplet surface (Dickinson, 2013; Douaire et al., 2014; Duffus et al., 2016; Ghosh et al., 2011; Ghosh and Rousseau, 2011; Haj-shafiei et al., 2013; Pickering, 1907; Ramsden, 1903; Rayner et al., 2014). The latter is commonly known as Pickering stabilization. Importantly, emulsifiers can play a role in promoting solid particle adsorption to surfaces by modifying the wettability of the particle itself (Binks, 2002; Chevalier and Bolzinger, 2013) or mediating interfacial crystallization by providing a non-polar droplet surface amenable for fat crystallization (Frasch-Melnik et al., 2010; Ghosh and Rousseau, 2012). The latter possibly involves templating or small crystals adsorbing to the surfactant monolayer and undergoing crystal growth, with both mechanisms providing the droplet with a mechanically rigid interface that is stable against coalescence (Douaire et al., 2014). In fat-continuous systems, monoacylglycerols (MAGs) are thought to facilitate interfacial crystal formation via heterogeneous nucleation at the droplet surface (Beri et al., 2013; Douaire et al., 2014; Frasch-Melnik et al., 2010; Ghosh and Rousseau, 2012; Krog and Larsson, 1992; Libster et al., 2009; Rafanan and Rousseau, 2017).

It is well-known that in the liquid state, incorporation of a dispersed phase within a continuous phase is likely to increase relative viscosity (Barnes, 1994). Furthermore, the extent to which dispersed droplets interact with the surrounding matrix can have a marked impact on rheology. When droplets reinforce material properties compared to an unfilled matrix, as denoted by an 
increase in elastic modulus $\left(\mathrm{G}^{\prime}\right)$ or viscosity, they are known as active fillers. Alternatively, if they weaken or cause no change, they are known as inactive fillers. Much of the work in this area has been carried out in oil-in-water $(\mathrm{O} / \mathrm{W})$ emulsion gels, where it has been found that filler activity depends on factors such as droplet surface characteristics, aggregation behaviour and average particle size and size distribution (Anton et al., 2001; Dickinson and Chen, 1999; Fröhlich et al., 2005; van Vliet, 1988). Filler "activity" is governed by different interparticle forces, depending on the nature of the continuous medium and the dispersed species. Attractive forces are mainly responsible for the increase in reinforcement seen with active fillers whereas repulsive forces are responsible for lack of change or weakening observed with inactive fillers. The forces governing viscosity and overall rigidity in colloidal dispersions also dictate the rheological behaviour of composite mixtures, namely Brownian motion, hydrodynamic forces and colloidal forces, which work in concert to give composites their material properties, depending on the shear stress (or rate) applied. Properties such as emulsion viscosity and elasticity can be further modified by altering the type, concentration and physical state of emulsifiers present at the oil-water interface.

Though less studied, similar findings have been found with water-in-oil (W/O) emulsions, where promotion of interactions between aqueous droplets and surrounding fat crystals can enhance emulsion elasticity whereas minimized droplet-fat crystal interactions yield little effect on rheology (Rafanan and Rousseau, 2017). For example, in microcrystalline wax systems, particlestabilized droplets were shown to exhibit higher $\mathrm{G}^{\prime}$ values over non-Pickering droplets (Le Révérend et al., 2011). In crude oil, asphaltene-resin colloids adsorbed at the interface have been shown to alter emulsion stability and flowability (Kumar et al., 2001).

Solid particles dispersed within a fat matrix, also considered a composite material, have been investigated as texture modifiers in food products, a prime example being chocolate where sugar, 
cocoa mass and potentially milk powder are dispersed in a continuous cocoa butter phase. Studies delving into particle size effects on molten chocolate flow properties have shown that a higher degree of particle interactions results in an increase in viscosity, but that increasing particle size counters this effect (Luckham and Ukeje, 1999). Specifically, the particle size distribution of solids dispersed in cocoa butter may enhance shear-thinning effect, depending on the particle size, the size distribution width, and fat content (Afoakwa et al., 2008; Do et al., 2007). Such studies demonstrate that control of particle-particle interactions in fat-continuous matrices may place a significant role in altering the rheology of the resulting composite.

Although fat crystal-stabilized emulsions are conceptually similar to composite materials containing solid particles, dispersed droplets are rarely thought of in the same light. The objective of the present study was to demonstrate how the presence of a dispersed aqueous phase significantly influences the viscoelastic properties of W/O emulsions, with a focus on the role of droplet size and distribution and extent of interactions with neighbouring droplets and fat crystals. Three emulsifiers yielding different interfacial characteristics were employed: glycerol monooleate (GMO) and glycerol monostearate (GMS) both promoted interfacial fat crystallization whereas polyglycerol polyricinoleate (PGPR) showed little influence. Emulsifiers were chosen not only for their ability to emulsify, but their ability to create a functional interface with attractive/repulsive qualities that are optimized with respect to droplet size distribution. Such an approach offers the opportunity to systematically enhance certain textural properties with minimal changes to ingredients, for a wide range of applications including low-fat spreads, cookie fillings, coatings and ganaches. 


\subsection{Materials and Methods}

\subsubsection{Materials}

All fat crystal-stabilized emulsions consisted of a $20 \mathrm{wt} \%$ aqueous phase dispersed in an $80 \mathrm{wt} \%$ oil phase made up of $14.4 \mathrm{wt} \%$ high-melting fat, $0.4-3.2 \mathrm{wt} \%$ emulsifier and 62.4-65.2 wt $\%$ canola oil (Table 3.1), with these compositions selected based on extensive preliminary experiments. All emulsions were made using one of three emulsifiers selected for this study: glycerol monooleate (GMO, Monomuls90-O18, BASF Canada, Mississauga, ON, Canada, HLB 3.4), glycerol monostearate (GMS, Alphadim SBK, Corbion, Mississauga, ON, Canada, HLB 3.8), or polyglycerol polyricinoleate (PGPR, Nealanders, Mississauga, ON, Canada HLB 3). Canola oil was purchased from a local supermarket (acid value $<0.2 \%$ ) and used without further purification whereas hydrogenated soy oil (HSO) was used as the high-melting fat (acid value $<0.02 \%$, bulk m.p. $72{ }^{\circ} \mathrm{C}$ of $\beta$ polymorph) and consisted primarily of tristearin (Bunge, Bradley, IL, USA).

Table 3.1- Emulsion and blank compositions (wt.\%). The aqueous phase consisted of $0.02 \mathrm{mM}$ Rhodamine $\mathrm{B}$ as dye and $5 \mathrm{mM} \mathrm{NaCl}$ to prevent double emulsification.

\begin{tabular}{ccccc}
\hline & Water (wt.\%) & HSO (wt.\%) & Emulsifier (wt.\%) & Canola oil (wt.\%) \\
\hline GMO & 20.0 & 14.4 & 1.6 & 64.0 \\
GMS & 20.0 & 14.4 & 3.2 & 62.4 \\
PGPR & 20.0 & 14.4 & 0.4 & 65.2 \\
GMO blank & 0.0 & 14.4 & 1.6 & 84.0 \\
GMS blank & 0.0 & 14.4 & 3.2 & 82.4 \\
PGPR blank & 0.0 & 14.4 & 0.4 & 85.2 \\
\hline
\end{tabular}




\subsubsection{Emulsion preparation}

The aqueous phase consisted of $0.05 \mathrm{M} \mathrm{NaCl}$ and $0.15 \mathrm{mM}$ Rhodamine. $\mathrm{NaCl}$ was added to prevent the formation of double emulsions during high pressure valve homogenization (Pradhan and Rousseau, 2012). Rhodamine allowed for better discernment of oil phase separation (oiling-off) $v s$ water separation as liquid phase pooling would be colourless in the case of oil migration and pink for aqueous separation. Given the viscosity of the emulsions after cooling and over time, separation may be seen in pockets throughout the storage vial, not necessarily localized at the bottom or top as is seen with less viscous liquid emulsions. The addition of $\mathrm{NaCl}$ and Rhodamine had no impact on emulsification droplet size, stability or interfacial characteristics based on preliminary tests. The experimental timeframe was 4 weeks, which consisted of weekly testing. Note that each emulsifier required different concentrations to produce kinetically stable emulsions with significantly different number-weighted droplet size (d50) values and narrow droplet size distributions (DSDs). Emulsions with either the smaller or larger d50 values are hereafter referred to as 'small DSD' and 'large DSD'.

\subsubsection{Monoacylglycerol-stabilized emulsions}

Two MAGs with different melting points were used: GMO (bulk m.p. $~ 35^{\circ} \mathrm{C}$ ) and GMS (bulk m.p. $\sim 60^{\circ} \mathrm{C}$ ). Two DSDs ('large' and 'small') were attained with each MAG by varying emulsification conditions (Table 2). Emulsion preparation required either a 3 or 4-step protocol depending on the desired average droplet size. For step 1, the oil phase components (fat, canola oil, and emulsifier) were heated, while stirring with a magnetic stir bar on a hot plate to $85{ }^{\circ} \mathrm{C}$ for $10 \mathrm{~min}$, to negate crystal history. The room temperature $\left(\sim 25^{\circ} \mathrm{C}\right)$ aqueous phase was added dropwise to the hot oil phase while heating to maintain the temperature of the resultant coarse 
emulsion at $85-90{ }^{\circ} \mathrm{C}$. With the aqueous phase added, the coarse emulsion was subjected to fine emulsification protocols which are detailed below and summarized in Table 3.2 (Steps $2-4$ ).

Table 3.2 - Homogenization conditions for MAG-stabilized emulsions to attain different droplet size distributions. Small and large distributions with the associated size are specified. Note that the diameter corresponding to "small" and "large" distributions are surfactant-specific. RPM are shown with units of relative centrifugal force $(\mathrm{x} \mathrm{g})$ given in brackets.

\begin{tabular}{|c|c|c|c|c|}
\hline & $\begin{array}{c}\text { Step 1: } \\
\text { Coarse } \\
\text { emulsion }\end{array}$ & $\begin{array}{c}\text { Step 2: } \\
\text { Fine emulsion } \\
\text { (rotor/stator) }\end{array}$ & $\begin{array}{c}\text { Step 3: } \\
\text { Fine emulsion } \\
\text { (valve } \\
\text { homogenizer) }\end{array}$ & $\begin{array}{c}\text { Step 4: } \\
\text { Shear-cooling }\end{array}$ \\
\hline $\begin{array}{c}\text { GMO, } \\
\text { Small } \\
\mathbf{d}_{50}=\mathbf{1} \boldsymbol{\mu m}\end{array}$ & $\begin{array}{l}10 \mathrm{~min}, 150 \mathrm{rpm} \\
(2 \times \mathrm{g}), 85^{\circ} \mathrm{C}\end{array}$ & $\begin{array}{c}8000 \mathrm{rpm}(900 \mathrm{x} \mathrm{g}), \\
3 \mathrm{~min}, 85^{\circ} \mathrm{C}\end{array}$ & 7000 psi & $\begin{array}{l}\text { Impeller, } 260 \mathrm{rpm} \\
\quad(5 \mathrm{x} \mathrm{g}), 13^{\circ} \mathrm{C}\end{array}$ \\
\hline $\begin{array}{c}\text { GMO, } \\
\text { Large } \\
\mathbf{d}_{50}=\mathbf{5} \boldsymbol{\mu m}\end{array}$ & $\begin{array}{l}10 \mathrm{~min}, 150 \mathrm{rpm} \\
(2 \mathrm{x} \mathrm{g}), 85^{\circ} \mathrm{C}\end{array}$ & - & 500 psi & $\begin{array}{l}\text { Impeller, } 260 \mathrm{rpm} \\
\quad(5 \times \mathrm{g}), 13{ }^{\circ} \mathrm{C}\end{array}$ \\
\hline $\begin{array}{c}\text { GMS, } \\
\text { Small } \\
\text { d50 }=\mathbf{1 0} \boldsymbol{\mu m}\end{array}$ & $\begin{array}{l}10 \mathrm{~min}, 150 \mathrm{rpm} \\
(2 \mathrm{x} \mathrm{g}), 85^{\circ} \mathrm{C}\end{array}$ & $\begin{array}{c}8000 \mathrm{rpm}(900 \mathrm{x} \mathrm{g}), \\
3 \mathrm{~min}, 85^{\circ} \mathrm{C}\end{array}$ & 7000 psi & $\begin{array}{l}\text { Impeller, } 260 \mathrm{rpm} \\
\quad(5 \mathrm{x} \mathrm{g}), 13{ }^{\circ} \mathrm{C}\end{array}$ \\
\hline $\begin{array}{c}\text { GMS, } \\
\text { Large } \\
\mathbf{d}_{50}=\mathbf{2 8} \boldsymbol{\mu m}\end{array}$ & $\begin{array}{l}10 \mathrm{~min}, 150 \mathrm{rpm} \\
(2 \mathrm{x} \mathrm{g}), 85^{\circ} \mathrm{C}\end{array}$ & $\begin{array}{c}8000 \mathrm{rpm}(900 \times \mathrm{g}), \\
3 \mathrm{~min}, 85^{\circ} \mathrm{C}\end{array}$ & 700 psi & $\begin{array}{l}\text { Impeller, } 260 \mathrm{rpm} \\
\quad(5 \mathrm{x} \mathrm{g}), 13^{\circ} \mathrm{C}\end{array}$ \\
\hline
\end{tabular}

The rotor/stator fitted with a two-blade aggregate (PT3100 Kinematica, Inc., Bohemia, NY, USA) was only used where indicated. Only the GMO-based emulsion with the larger DSD bypassed use of the rotor/stator. High-pressure homogenization (APV-1000, APV, Albertslund, Denmark) was performed continuously for $3 \mathrm{~min}$ at the different pressures specified in Table 2. All MAGstabilized emulsions were shear-cooled in the same manner, which consisted of cooling the 
emulsion in a metal beaker partially submerged in an ice bath while shearing using an overhead impeller to solidify the HSO and MAG components. Once the hot emulsion was transferred to the metal beaker, the overhead stirrer attached to a three-finned impeller blade (Stirrer: Model BDC3030, Blade: AC533, Caframo Lab Solutions Ltd., Georgian Bluffs, ON, Canada) was lowered into the beaker to provide shear at $260 \mathrm{rpm}(5 \mathrm{x} \mathrm{g} \mathrm{RCF})$ until a temperature of $13{ }^{\circ} \mathrm{C}$ was reached $(\sim 5 \mathrm{~min})$. The cooled emulsions were then transferred to containers, sealed and stored at

$4{ }^{\circ} \mathrm{C}$. The control samples consisted of fat, oil and emulsifier where the aqueous phase was replaced with an equal mass of canola oil; their processing and HSO content was as per the corresponding emulsions.

\subsubsection{PGPR-stabilized emulsions}

As generating PGPR-stabilized emulsions with highly-controllable DSDs consisting of a larger average droplet size proved challenging, the fat phase was pre-crystallized without emulsifier and then emulsification was carried out with PGPR and the aqueous phase (Table 3.3). The HSO and canola oil were heated to above $80{ }^{\circ} \mathrm{C}$ and stirred continuously over for $10 \mathrm{~min}$. The hot oil-fat mixture was then transferred to the shear-cooling apparatus and sheared via impeller to a temperature of $14^{\circ} \mathrm{C}$ as described above. The emulsifier was added and sheared for $10 \mathrm{~s}$ to ensure incorporation. The room temperature aqueous phase was added dropwise and sheared in the cooling apparatus with the impeller at $260 \mathrm{rpm}(5 \mathrm{x} \mathrm{g}$ RCF) to achieve the small DSD and $85 \mathrm{rpm}$ $(<1 \mathrm{x} \mathrm{g}$ RCF) to produce the large DSD. Once the aqueous phase was fully added, the emulsion was sheared for one additional min to ensure that all water had been emulsified. Samples were then transferred to storage containers, sealed and stored at $4{ }^{\circ} \mathrm{C}$. The PGPR controls consisted of 
the oil, fat and emulsifier processed in the same manner as the emulsions with the aqueous portion replaced by canola oil.

Table 3.3 - Homogenization conditions for PGPR-stabilized emulsions to attain different droplet size distributions. Small and large distributions with the associated size are specified. Note that the diameter corresponding to "small" and "large" distributions are surfactant-specific. RPM are shown with units of relative centrifugal force $(\mathrm{g})$ given in brackets.

\begin{tabular}{cccc}
\hline & $\begin{array}{c}\text { Step 1: } \\
\text { Pre-crystallization } \\
\text { of molten HSO-oil } \\
\text { mix }\end{array}$ & $\begin{array}{c}\text { Step 2: } \\
\text { Introduction of } \\
\text { PGPR to crystallized } \\
\text { HSO + oil mix }\end{array}$ & $\begin{array}{c}\text { Step 3: } \\
\text { Emulsification } \\
\text { (impeller mixing) }\end{array}$ \\
\hline $\begin{array}{c}\text { PGPR, Small } \\
\text { d50 }=\mathbf{1 0} \boldsymbol{\mu m}\end{array}$ & $\begin{array}{c}\text { Cool from } 85{ }^{\circ} \mathrm{C} \text { to } \\
14{ }^{\circ} \mathrm{C}\end{array}$ & $10 \mathrm{~s}, 14^{\circ} \mathrm{C}$ & $260 \mathrm{rpm}(5 \mathrm{x} \mathrm{g}), 14{ }^{\circ} \mathrm{C}$ \\
PGPR, Large & & & $85 \mathrm{rpm}(<1 \mathrm{x} \mathrm{g}), 14$ \\
d50 $=\mathbf{2 6} \boldsymbol{\mu m}$ & & & ${ }^{\circ} \mathrm{C}$ \\
\hline
\end{tabular}

\subsubsection{Solid fat content and droplet diameter with pulsed NMR}

Droplet size and solid fat content (SFC) were determined by pulsed NMR. Immediately after preparation, control and emulsion samples were pipetted into NMR tubes to heights of $1 \mathrm{~cm}$ and 6 $\mathrm{cm}$, respectively. Samples were subsequently stored at $4{ }^{\circ} \mathrm{C}$ until analysis and re-tested weekly. The SFC of the emulsions was determined at $4{ }^{\circ} \mathrm{C}$ using a temperature-controlled Bruker Minispec Mq20 (Bruker Canada, Milton, ON, Canada) with the application sfc_lfc v2.51 whereas the d50 values were determined using the water droplet v2.51 application. When droplet sizes were below 
the threshold for NMR determination of droplet size (approximately $1 \mu \mathrm{m}$ ), image analysis was used as described below.

\subsubsection{Microscopy}

The morphology of the fat crystal network and dispersed aqueous droplets were examined by brightfield and polarized light microscopy. All samples were characterized at room temperature using a Zeiss Axiovert 200M inverted light microscope at 630 x magnification. Emulsion droplet diameters too small to reproducibly size by NMR were determined through image analysis using ImageJ software (v1.51, Research Service Branch, National Institute of Health, Bethesda, MD), using protocols indicated in our previous research (Rafanan and Rousseau, 2017). The background subtraction was performed on 8-bit images, which were converted to binary. Droplet diameter was measured using the "Analyze particles" macro, native to ImageJ. A frequency distribution of the measured particle diameters was created for each image. A minimum of 10 images was used per trial $(n=3)$. The population median was used as the small droplet size value as an approximation of the d50 value.

\subsubsection{Wide angle $x$-ray diffraction}

Fat crystal polymorphic behaviour over time was determined using wide-angle x-ray diffraction (WAXD) of the emulsions using a Hecus S3-MICROcaliX X-ray diffractometer (Hecus X-ray systems, Graz, Austria). Approximately $1 \mathrm{mg}$ samples were loaded into $1.0 \mathrm{~mm}$ quartz capillaries

to a height of $1 \mathrm{~cm}$ and $\mathrm{x}$-ray signals were captured for 10 min given the weak signal produced by the low-fat emulsions. The $\mathrm{x}$-ray source was provided by $50 \mathrm{kV}, 1 \mathrm{~mA} \mathrm{KCu}$ anode and collimated 
with a slit collimator. WAXD scanning range was from $\theta=9.4-14.4^{\circ}$, corresponding to d-spacing values of between $3.1-4.8 \AA$. Samples in quartz capillaries were stored at $5{ }^{\circ} \mathrm{C}$ until the next sampling interval.

\subsubsection{Rheology}

For the emulsion linear viscoelastic region range (LVR) and corresponding storage modulus $\left(\mathrm{G}^{\prime}\right)$, amplitude sweeps were performed at $5{ }^{\circ} \mathrm{C}$ on an Anton-Paar MCR301 rheometer (Anton Paar, St. Laurent, QC, Canada) equipped with a temperature-controlled, Teflon-coated Peltier stage and a parallel plate geometry (PP25 mm diameter, Teflon) set at a gap of $0.5 \mathrm{~mm}$. Amplitude sweeps were conducted at $1.6 \mathrm{~Hz}$ from $0.01-100 \%$ strain at $5{ }^{\circ} \mathrm{C}$. The LVR cut-off was determined as the strain value where sample $\mathrm{G}^{\prime}$ deviated no more than $5 \%$ from its ordinate value. Viscosity measurements were conducted with the same geometry, gap and temperature with the rotational shear rate in a continuous ramp increasing from $0-100 \mathrm{~s}^{-1}$.

\subsubsection{Data analysis}

One-way and two-way ANOVAs were performed on amplitude sweep and viscosity data using SigmaPlot 11 (Systat Software Inc., San Jose, CA, USA). Viscosity curves were fit to a power law distribution using the CurveFit GUI available through MatLab (Version R2016a, Mathworks Inc. Natick, MA, USA). Triplicate experiments and measurements were taken for all treatments $(n=3)$. 


\subsection{Results and Discussion}

\subsubsection{Visual appearance and dispersed phase droplet size}

The PGPR emulsions (i.e., with smaller and larger average d50 values) were viscous, pourable liquids (Figure 3.1). By contrast, all MAG-stabilized emulsions were soft, paste-like solids that retained their shape. The MAG-based, as well as the small DSD PGPR emulsion, were visually homogeneous with no evidence of phase separation over the 28-day experimental period. The large DSD PGPR emulsion, however, began to phase-separate by Day 14, with evidence of aqueous phase pooling at the bottom of the storage vial thereafter. As a result, its characterization took place for only 14 days. The d50 of the small and large DSDs for both GMS and PGPR-stabilized emulsions were centered at $\sim 10$ and $26-28 \mu \mathrm{m}$, respectively. The GMOstabilized emulsions, however, displayed small and large average d50 values of $\sim 1$ and $\sim 5 \mu \mathrm{m}$, respectively. No significant increase in droplet diameter was observed, indicating that all stable emulsions did not undergo coalescence for least 28 days ( $p>0.05$ ). 

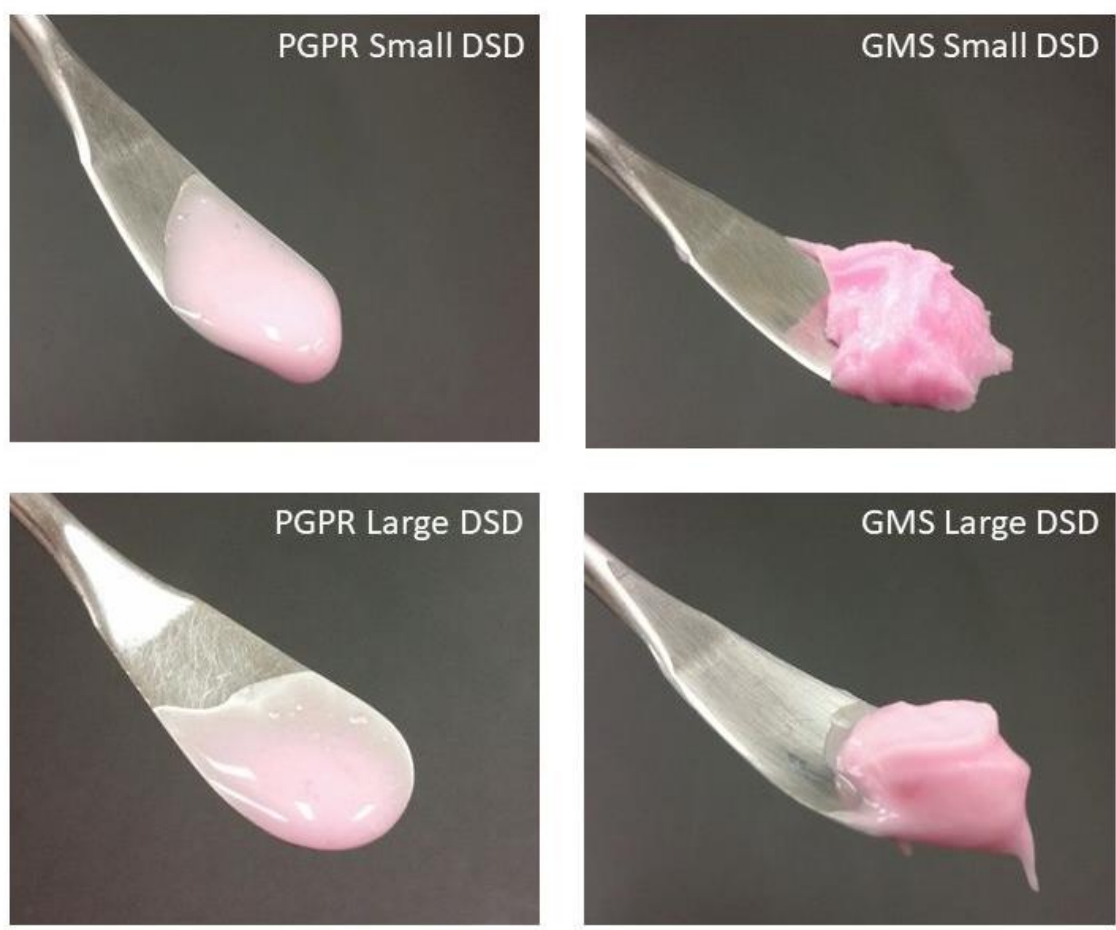
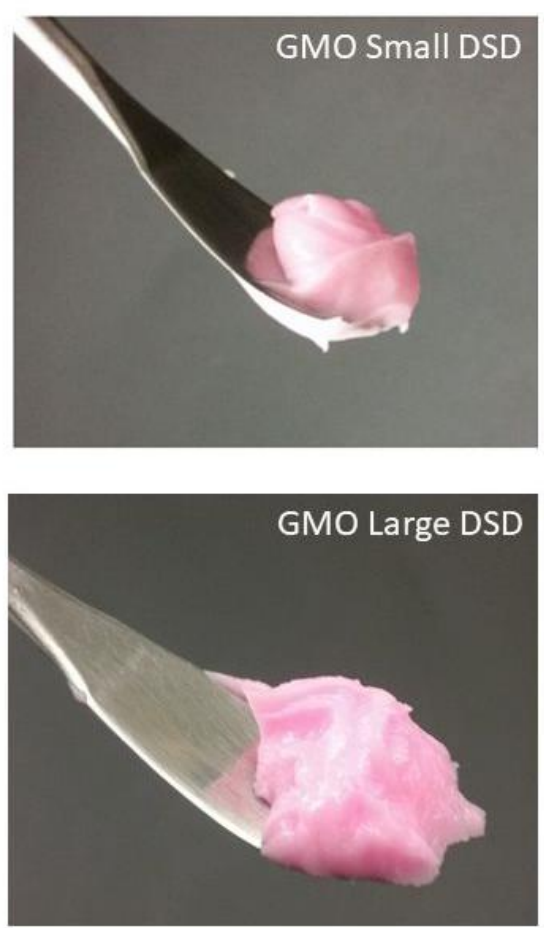

Figure 3.1 - Visual appearance of the different fat crystal-stabilized emulsions. The top row shows emulsions with small average droplet sizes whereas the bottom row shows emulsions with the larger average droplet sizes.

To establish emulsions with unique rheological properties, three emulsifiers giving different interfacial characteristics were chosen, with both GMO and GMS promoting interfacial crystallization of HSO and PGPR not doing so. Furthermore, different homogenization protocols were used to create emulsions yielding distinct d50 values for each emulsifier using a single concentration. Preparation of the MAG-based emulsions was straightforward with different droplet sizes obtained for each emulsifier. However, finding a single PGPR concentration resulting in emulsions with distinct $\mathrm{d} 50$ values was challenging as forming a stable emulsion with a larger d50 value (e.g., $\sim 26 \mu \mathrm{m}$ ) was not possible. This was unusual as PGPR is a known, highly efficient emulsifier that generates emulsions with small d50 values at low concentrations $(<1 \mathrm{wt} \%)$, owing 
to its ability to produce a thick elastic interface (Beri et al., 2013; Dedinaite and Campbell, 2000).

The presence of pre-crystallized fat hampered the otherwise excellent ability of PGPR to effectively disperse the aqueous phase into small droplets.

\subsubsection{Fat crystal network properties}

The SFC of all emulsions remained the same over the 28-day experimental timeframe. The SFC of the GMS-stabilized emulsions was higher than either the GMO or PGPR-stabilized emulsions ( $17.5 \%$ for GMS versus $\sim 14.5 \%$ and $\sim 15.5 \%$ for PGPR and GMO, respectively) due to the higher wt\% GMS required to prevent an increase in droplet size or oil/water phase separation. The SFC of the GMS emulsions and control corresponded to the sum of the wt\% of crystalline GMS and HSO. Lack of change in SFC over time in any emulsion suggested a lack of fat post-crystallization events $(\mathrm{p}>0.05)$

Figure 3.2 compares brightfield and polarized light images of all emulsions following their preparation. The upper panel in Figure 3.2, which shows brightfield images, demonstrated the difference in droplet size for all 3 emulsifiers. Regardless of emulsifier type or emulsification method, HSO crystal aggregates appeared as large, smooth and ovoid-shaped with long axes measuring 10 - $40 \mu \mathrm{m}$ in length (Figure 3.2A-F, arrows), and did not change over time. Their shape was induced by shearing, which collected and compacted neighbouring fat crystals into aggregates. Both MAG-stabilized emulsions, irrespective of average d50, showed evidence of interfacial crystallization, with boxed areas (Figures 3.2b, c, e and f) showing crystallized 'rings' at the droplet surface under polarized light. By contrast, there was absence of a crystalline interface in the PGPR-based emulsions (Figure 3.2a and d). Rather, the interface of these droplets was smooth 
and only the fat crystal aggregates transmitted polarized light. The absence of interfacial crystallization in the presence of PGPR has been observed in other studies (Nadin et al., 2014; Rafanan and Rousseau, 2017; Tran et al., 2017), and has been attributed to the branched, disordered structure of the emulsifier at the interface limiting TAG adsorption to the droplet surface (Ghosh and Rousseau, 2012). These features did not change during storage. 

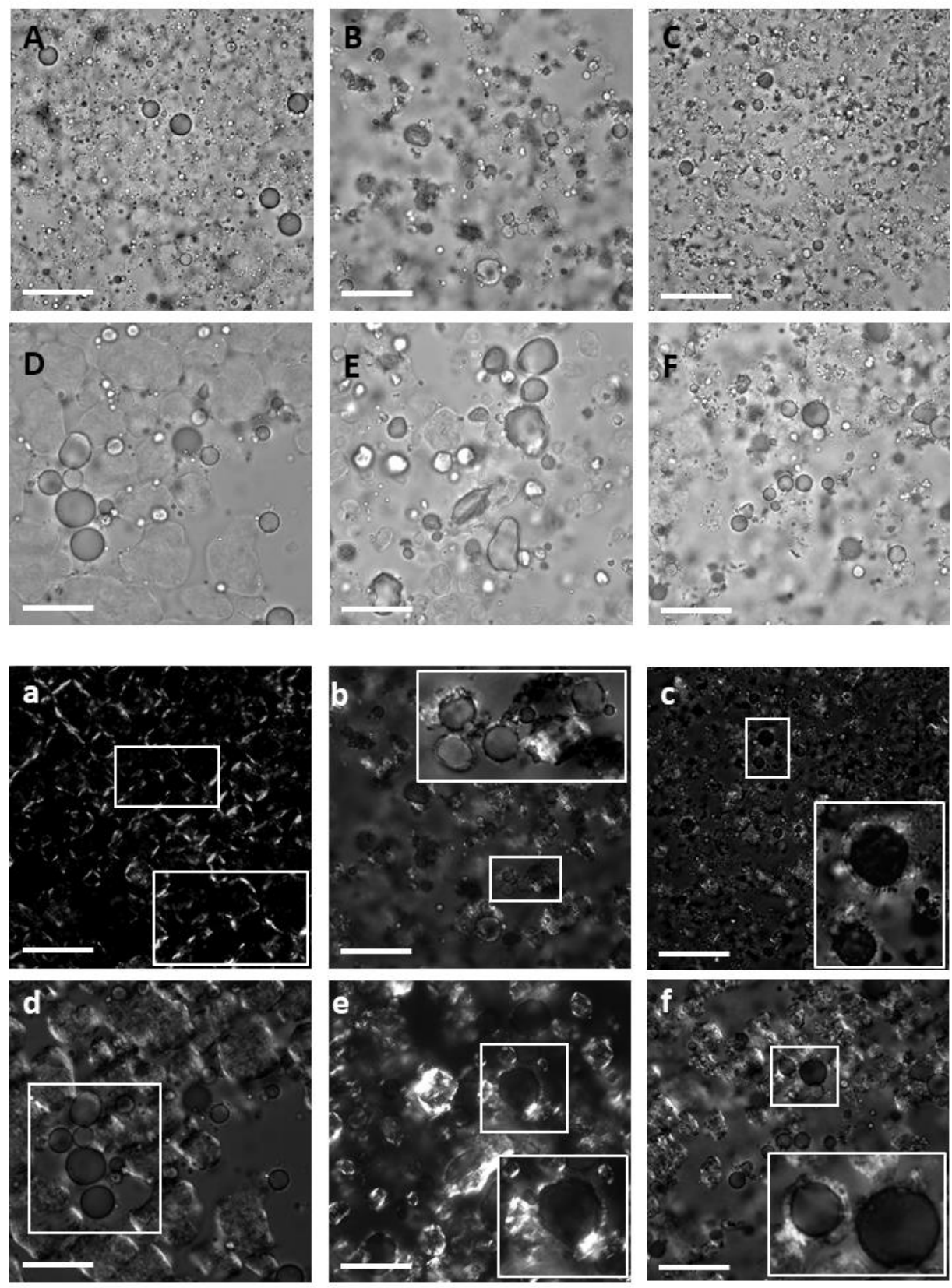

Figure 3.2 - Brightfield (upper panel) and polarized light (lower panel) microscopy of fat crystalstabilized emulsions prepared with PGPR, GMS, or GMO. PGPR small (A, a), GMS Small (B, b), GMO Small (C, c), PGPR Large (D, d), GMS Large (E, e), GMO Large (F, f). White arrows in the brightfield panels (A-E) indicate HSO aggregates comprising the fat crystal network. Scale bar is $50 \mu \mathrm{m}$. 
Table 3.4 displays the polymorphic behaviour of the fat phase present in all emulsions (polymorph identified from WAXS peaks in Appendix figure 3.1). The reason for identifying TAG crystal form was to assess whether the presence of dispersed droplets impacted fat crystal polymorph, given the propensity of emulsifiers to preferentially partition to the droplet surface. In the absence of dispersed droplets, emulsifiers are much more apt to be integrated within the lattice structure of TAG crystals. Comparison of the water-free blank and emulsions helped to highlight whether there were any differences in polymorphic form due to the presence of dispersed droplets.

Table 3. 4 - Dominant TAG crystal polymorphs identified in emulsions made with HSO and either GMS, PGPR or GMO over time. Two average droplet sizes and the water-free control are represented for each emulsifier. * indicates that data is valid for only days 0-14.

\begin{tabular}{ccccc}
\hline & Days & GMS & PGPR & GMO \\
\hline \multirow{2}{*}{ Control } & $0-21$ & $\beta^{\prime}$ & $\beta$ & $\beta+\beta^{\prime}$ \\
& 28 & $\beta^{\prime}+\beta$ & $\beta$ & $\beta+\beta^{\prime}$ \\
\hline Small & 0 & $\beta^{\prime}+\beta$ & $\beta$ & $\beta+\beta^{\prime}$ \\
DSD & $7-28$ & $\beta$ & $\beta$ & $\beta+\beta^{\prime}$ \\
\hline Large & 0 & $\beta^{\prime}>>\beta$ & $\beta$ & $\beta+\beta^{\prime}$ \\
DSD & $7-28$ & $\beta^{\prime}>\beta$ & $\beta^{*}$ & $\beta+\beta^{\prime}$ \\
\hline
\end{tabular}

From the outset, the HSO in the PGPR-based emulsions and control existed in the $\beta$-polymorph based on WAXD peaks at 3.7, 3.9 and $4.6 \AA$. This was expected due to the HSO crystallization prior to emulsification. Given its high tristearin content, HSO readily forms $\beta$-crystals with rapid cooling (Lutton, 1945).

The GMS control consisting of a mixture of HSO and GMS in canola oil exhibited a slow transition from the $\beta^{\prime}$ to the $\beta$-polymorph, which eventually appeared by Day 28 . This was due to the ability of GMS to slow and/or hinder the $\beta^{\prime}$-to- $\beta$ transition of saturated TAGs (Aronhime et al., 1988). 
Once emulsified, a different behaviour was observed, suggesting that presence of the droplet interface altered the ability of GMS to modify HSO polymorphic behaviour, as the emulsifier was preferentially adsorbed to the droplet surface rather than present in the bulk. Only the GMSstabilized emulsions showed evolution in subcell arrangement as a function of droplet size. On day 0 , a mix of $\beta^{\prime}$ and $\beta$ crystals was present in both emulsions. The small DSD GMS emulsions initially existed in a mix of $\beta^{\prime}$ and $\beta$-crystals based on the presence of spacings at 3.8 and $4.2 \AA$ as well as 3.7, 3.9, and $4.6 \AA$, respectively. Over time, there was partial loss of $\beta^{\prime}$ character based on the disappearance of the peak at 3.8 $\AA$. The large DSD GMS emulsion also existed in a mix of $\beta^{\prime}$ and $\beta$ polymorphs on Day 0 , based on the presence of spacings at 3.8 and $4.2 \AA$ as well as $4.6 \AA$ respectively. Over time, this emulsion retained much of its $\beta^{\prime}$ character, though the appearance of spacings at 3.7 and $3.9 \AA$ by Day 7 indicated increased presence of $\beta$-crystals.

The HSO in the GMO control, as well as small and large DSD emulsions, exhibited mostly $\beta$ character, with a persistent, strong peak at $4.6 \AA$, though there was a broad, weak peak at $\sim 3.8 \AA$ associated with $\beta^{\prime}$ crystals. This was due to the recognized ability of GMO to produce mixed crystals in the presence of saturated TAGs and palm fat blends (Shiota et al., 2011; Tran et al., 2017). These peaks did not evolve during storage.

It is well-known that emulsifiers may significantly impact fat TAG nucleation, crystal growth or both, depending on their composition, added amount and mechanism of action (Smith et al., 2011). For this to occur, however, the emulsifier must be sufficiently mobile and demonstrate some molecular complementarity to be incorporated into a TAG's crystal lattice. If adsorbed to an oilwater interface, emulsifiers will no longer be as available for integration into a growing fat crystal lattice, as would occur in the bulk. Therefore, GMS, which delayed the $\beta^{\prime}$-to- $\beta$ transition of HSO in the emulsion-free control (Table 3.4), showed a limited capacity to do so once adsorbed to an 
interface. After 4 weeks, there was presence of $\beta$ crystals in all GMS-based systems. However, the absence of dispersed droplets (i.e., in the control) resulted in the slowest $\beta^{\prime}$-to- $\beta$ transition followed by the large droplets and finally the small droplets which mediated a faster, more extensive polymorphic transition from $\beta^{\prime}$ to $\beta$-crystals, as observed in other fat-emulsifier systems (Aronhime et al., 1988). Therefore, presence of an oil-water interface sequestered the GMS away from the bulk fat thereby altering the polymorphic behaviour of HSO in bulk vs. emulsions.

Neither PGPR nor GMO exhibited this behaviour. In the case of PGPR, the lack of polymorphic transition was due to the addition of PGPR after crystallization of HSO resulting in $\beta$-crystal formation during preparation. GMO promoted formation of a mix of $\beta$ and $\beta^{\prime}$ by disrupting unit cell packing (Smith et al., 2011), though this behaviour has yet to be validated in many fats.

\subsubsection{Rheological profiles}

\subsubsection{Amplitude sweep of fat crystal-stabilized emulsions}

Figure 3.3 shows the elastic behaviour $\left(\mathrm{G}^{\prime}\right.$ values in $\left.\mathrm{kPa}\right)$ for all fat crystal-stabilized emulsions and associated controls as a function of time. Comparing Figures 3.3A with $3.3 \mathrm{~B}$ and $3.3 \mathrm{C}$, the PGPR-based emulsions expectedly showed much lower G' values than the GMS and GMO-based emulsions, in line with the observed consistency (Figure 3.3A). Throughout the experimental timeframe, the PGPR control maintained $\mathrm{G}^{\prime}$ values of $\sim 0.05 \mathrm{kPa}$. On day 0 , the $\mathrm{G}^{\prime}$ values of the small and large DSD PGPR emulsion were $\sim 2$ and $\sim 6 \mathrm{kPa}$, respectively. After day 7, both $\mathrm{G}^{\prime}$ values decreased to $<0.5 \mathrm{kPa}$, in line with the PGPR control ( $>>0.05$ ). 

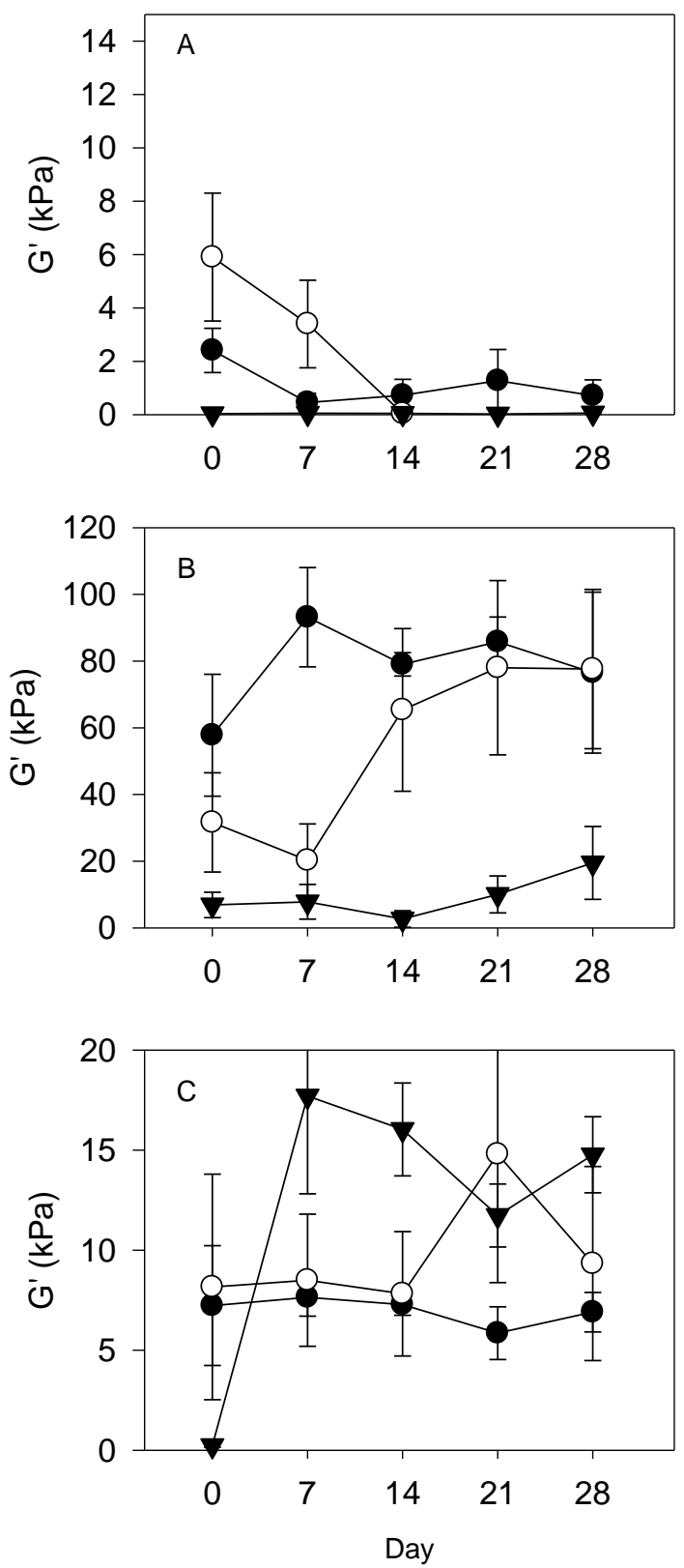

Figure 3.3 - Evolution in $\mathrm{G}^{\prime}(\mathrm{kPa})$ of emulsions made with PGPR (A), GMS (B) or GMO (C). Droplet size distributions are shown as (Small DSD), $\bigcirc$ (Large DSD), and $\nabla$ (control). Note that PGPR Large DSD data is only available up to Day 14 as the emulsions phase separated thereafter. Error bars show standard error.

Figure 3.3B shows that both large and small DSD GMS-stabilized emulsions not only had the highest $\mathrm{G}^{\prime}$ values overall but displayed the greatest sensitivity to droplet size. The small DSD 
emulsion consistently exhibited higher $\mathrm{G}^{\prime}$ values over the control and large DSD emulsion. The small DSD emulsions reached a value of $\sim 93 \mathrm{kPa}$ at Day 7 and appeared to plateau. The large DSD reached a plateau after Day $14(\sim 65 \mathrm{kPa})$. The $\mathrm{G}^{\prime}$ value of the GMS control continued to slowly increase over time, reaching a maximum value of $\sim 20 \mathrm{kPa}$ on day 28 .

Finally, both GMO emulsions (Figure 3C) were soft solids and exhibited higher $\mathrm{G}^{\prime}$ values than the PGPR-stabilized emulsions. On day 0, the GMO-containing control was liquid-like with a G' value $<1 \mathrm{kPa}$, indicating that the presence of droplets promoted elastic behaviour immediately after shear-cooling. However, from day 7 onwards, its $\mathrm{G}^{\prime}$ value exceeded that of both emulsions $(\sim 18$ $\mathrm{kPa}$ ) and had a soft solid texture. For all GMO-based samples, $\mathrm{G}^{\prime}$ values were stable after Day 7 , measuring 8 - $9 \mathrm{kPa}$ for all emulsions. In the absence of water droplets, post-crystallization processes appeared to be significantly slower, causing $\mathrm{G}^{\prime}$ to increase more gradually over time. Since there was no change in fat crystal polymorph or increase in SFC, increases in $\mathrm{G}^{\prime}$ were attributed to sintering of adjacent fat crystal aggregates (Macierzanka et al., 2009).

\subsubsection{Reinforcement index}

To better visualize how fillers in composite materials reinforce structure, one can use the ratio of the $\mathrm{G}^{\prime}$ of the composite material to that of the matrix without particles (Dickinson and Chen, 1999). In our case, the reinforcement index $(R)$ value provided a scale of how the presence of dispersed water droplets increased or decreased the elasticity of the HSO network (unfilled network) vs. fat crystal-stabilized emulsions (composite material):

$$
\log R=\frac{G_{\text {filled network emulsion }}^{\prime}}{G_{\text {Unfilled network }}^{\prime}}
$$


The $R$ value of the water-free, emulsifier-containing HSO network was also calculated to show how presence of an emulsifier in the bulk impacted fat crystal network reinforcement. As the log of the ratio is taken, $R>0$ indicates a strengthening of the material whereas $R<0$ denotes structural destabilization or weakening of the network. The average $\mathrm{G}^{\prime}$ values at $0.02 \%$ strain (which was within the LVR) were used for all $R$ calculations.

Figure 3.4A shows that addition of PGPR weakened the fat crystal network strength of HSO as $R$ values were below 0 at all time points. PGPR presumably reduced attractive interactions between adjacent HSO crystals, as has been observed in confectionery systems (Middendorf et al., 2015). This has been attributed to the looping ricinoleate branches in PGPR creating a thick and disordered interface that prevent close contact between neighbouring particles (Dedinaite and Campbell, 2000; Norton et al., 2009). The presence of water droplets strengthened the HSO network, with larger water droplets having a greater reinforcing effect over smaller droplets. It was expected that with their higher surface-to-volume ratio than larger droplets, the smaller droplets would create more contact points between adjacent droplets. However, this was not the case as the large DSD emulsions exhibited higher $R$-values at all time points. The increase in the $R$-values of the emulsions over the control was due to the volume fraction effect, with weakly-interacting dispersed droplets marginally increasing gel strength (Barnes, 1994). 

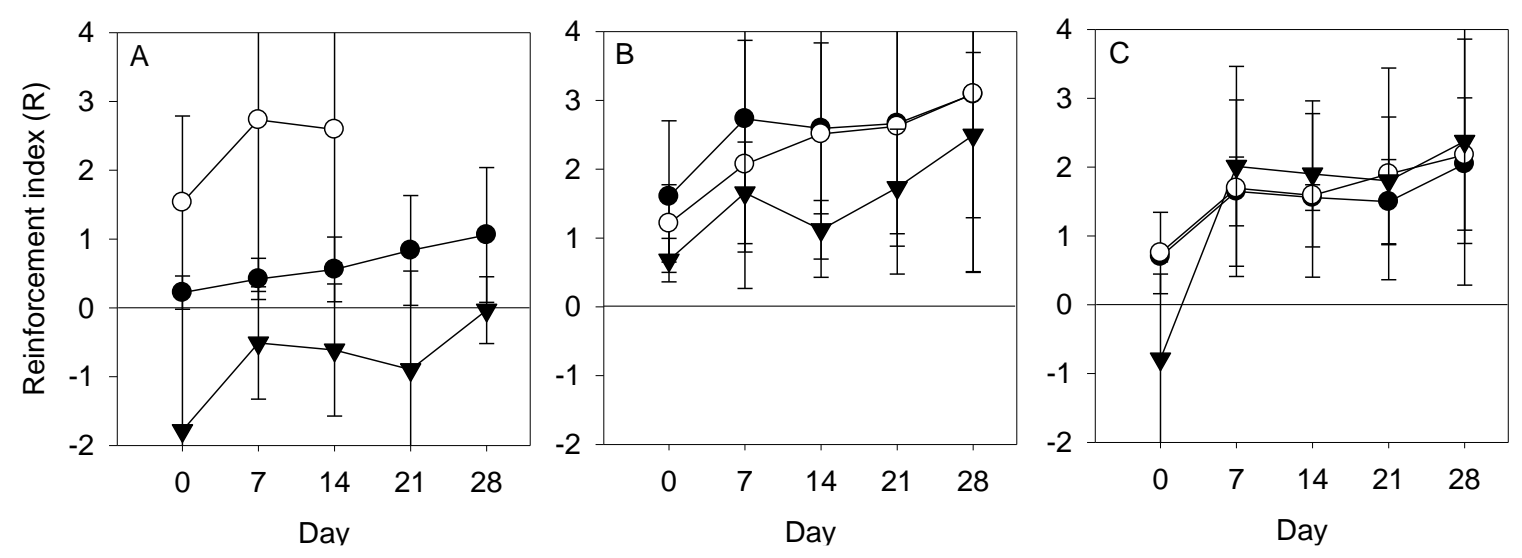

Figure 3.4 - Evolution in Reinforcement index (R) of emulsions made with PGPR (A), GMS (B) or GMO (C) over time. Droplet size distributions are shown as (Small DSD), $\bigcirc$ (Large DSD), and $\boldsymbol{\nabla}$ (control). A line shown at $\mathrm{y}=0$ identifies the transition between reinforcement $(\mathrm{R}>0)$ or weakening $(\mathrm{R}<0)$. Note that PGPR Large DSD data is only available up to Day 14 as the emulsions phase separated thereafter. Error bars show standard error.

The $R$-values for emulsions prepared with GMS showed network reinforcement $(R>0)$ with both the large and small DSDs (Figure 3.4B). With the small DSD, emulsions reached a maximum $R$ value after day 7 and then essentially plateaued whereas the $R$ value of the large DSD emulsion continued to gradually increase during the experimental timeframe. The $R$ values of both GMS emulsions were statistically similar ( $>>0.05)$, demonstrating that droplet size was unrelated to the maximum reinforcement of the HSO network. The GMS control did not reach the same plateau value as the emulsions and rose slowly throughout the experimental timeframe.

The similar trends in both $\mathrm{G}^{\prime}$ and $R$ likely resulted from the close packing exhibited by the small DSD emulsion droplets, which formed a network of aggregates within the interstices between fat crystal aggregates (e.g., Figures 3.2B and b). As the strength and number of the particle junction zones ultimately determines the reinforcement of the network, the observed increases in $\mathrm{G}^{\prime}$ and $R$ resulted from the greater numbers of such junctions. 
With GMO, the $R$ value of the control was initially below 0 (day zero), which indicated structural weakening. Between days 7 and 28, both GMO-based emulsions as well as the control approached similar $R$ values. Therefore, presence of a dispersed phase did not greatly alter the $R$ values of the emulsions. In fact, after day 7, the $R$ value of the GMO control surpassed that of both emulsions, although not significantly, suggesting that GMO adsorbed to the fat crystal aggregate surface and promoted sintering with ageing.

\subsubsection{Viscosity power law fitting}

Figure 3.5 shows typical apparent viscosity profiles at high and low shear rates $\left(100 \mathrm{~s}^{-1}\right.$ and $1 \mathrm{~s}^{-1}$, respectively) to highlight the change in viscosity as related to presence of a dispersed phase and average droplet size. All emulsions studied exhibited similar shear-thinning behaviour. Viscosity curve trends were summarized using the power law relationship (Do et al., 2007) (Equation 3.1):

$$
\eta=a \gamma^{b-1}
$$

where $\eta$ is viscosity $(\mathrm{Pa} \cdot \mathrm{s})$, a is the consistency index $\left(\mathrm{Pa} \cdot \mathrm{s}^{\mathrm{n}}\right), \gamma$ is shear rate $\left(\mathrm{s}^{-1}\right)$ and $\mathrm{b}$ is a unitless power law index. Fitting the viscosity data to a power law distribution linearizes the data to identify two factors: consistency ( $a$ coefficient) and shear-dependent changes in viscosity ( $b$ coefficient). Materials with higher values of $a$ will have a more solid-like character whereas lower values indicate a more liquid-like nature. The $b$ coefficient indicates shear-dependent deformation that occurs with increasing shear rate. As $b=1$ for Newtonian fluids, a value less than 1 indicates shear-thinning behaviour, with lower values indicating greater shear-thinning. For shearthickening materials, $b>1$. All fits discussed below had high $\mathrm{R}^{2}$ values $\left(\mathrm{R}^{2}>0.90\right)$. A table summarizing the $a$ and $b$ coefficients are included in the appendix as Appendix Tables 3.1 and 3.2. 
On day 0, both the large and small DSD PGPR-based emulsions displayed the lowest apparent viscosities at high and low shear $\left(100 \mathrm{~s}^{-1}\right.$ and $\left.1 \mathrm{~s}^{-1}\right)$ of all emulsions tested (Figures 3.5A vs. 3.5B and 3.5C). At $1 \mathrm{~s}^{-1}$, apparent viscosities ranged from 23 to $59 \mathrm{~Pa} \cdot \mathrm{s}$ for the large DSD and from 16 to $21 \mathrm{~Pa} \cdot \mathrm{s}$ for the small DSD PGPR emulsions. The high shear viscosity values for both emulsions were $~ 2 \mathrm{~Pa} \cdot \mathrm{s}$. Both emulsions showed higher viscosities than the PGPR control whose viscosity at low and high shear rates was $<2$ and 4 to $8 \mathrm{~Pa} \cdot \mathrm{s}$, respectively.
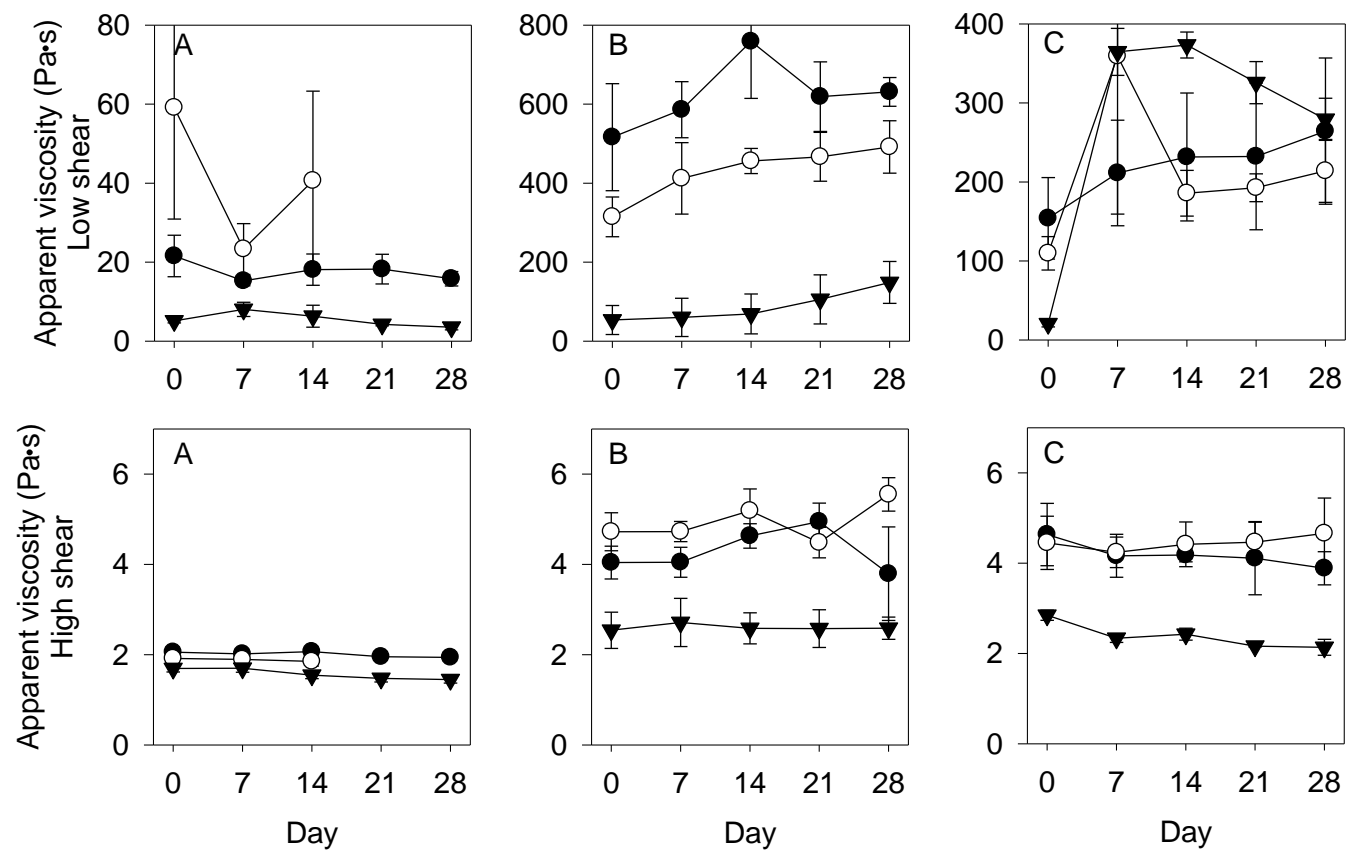

Figure 3.5 - Apparent viscosity at low shear ( $1 \mathrm{~s}^{-1}$, top row) and high shear (100 $\mathrm{s}^{-1}$, bottom row). Emulsion labels are as follows: PGPR (A), GMS (B) or GMO (C). Droplet size distributions are shown as (Small DSD), $\bigcirc$ (Large DSD), and $\nabla$ (control). Standard error bars shown. Note that the $y$-axis maxima of the top row graphs are not set to the same scale.

Of all samples tested, those containing PGPR exhibited the lowest $a$ coefficients. The control displayed $a$ values ranging from 3 to $7 \mathrm{~Pa} \cdot \mathrm{s}$ whereas the small DSD PGPR emulsion $a$ values 
remained similar over time, ranging from 15 to $19 \mathrm{~Pa} \cdot \mathrm{s}$. Although the large DSD PGPR emulsion did not display a definite trend, $a$ values ranging from 22 to $55 \mathrm{~Pa} \cdot \mathrm{s}$ were consistently higher than the control and small DSD. The $b$ coefficients of the control were highest at $0.6-0.8$ vs the small and large DSD emulsion b values, which ranged from 0.4 to 0.8 and -1 to 6 , respectively. These results signified that for PGPR-stabilized droplets, larger droplet sizes promote an increase in viscosity, giving thicker consistencies.

The GMS-containing samples exhibited the highest apparent viscosities of all samples tested. The low-shear range for the blank, small and large DSD were 21 to 37, 516 to 759, and 315 to 492 Pa's, respectively. The high-shear viscosity values were $\sim 3, \sim 4$, and $\sim 5$ Pa's for the blank, small and large distributions, respectively.

The $a$ values for GMS small DSD emulsions did not change significantly during storage and displayed no trend with time, with values ranging from 461 to $670 \mathrm{~Pa} \cdot \mathrm{s}$ (p>0.05). The large GMS droplets gave lower values (271 - $422 \mathrm{~Pa} \cdot \mathrm{s})$, but also did not change significantly (p>0.05). The $b$ values of both emulsions overlapped at approximately -0.3 and did not change during storage, indicating that although droplet size influenced consistency in the GMS-stabilized emulsions, it did not affect shear-thinning behaviour. The GMS control exhibited no change in $b$ from day 0 to 7, but did decrease consistently until Day 28 at which point it was less than both emulsions.

Both large and small GMS emulsions deformed at the same rate (similar $b$ coefficients), but the small GMS DSD emulsions had a more solid-like structure at lower shear, which was attributed to the more numerous inter-droplet connections present. At higher shear, these interparticle bonds broke, allowing the large and small droplet emulsions to flow similarly. Therefore, the number of 
interparticle bonds (i.e., small droplet interactions), was primarily responsible for overall consistency in GMS-stabilized emulsions.

The low-shear apparent viscosity range for the GMO-containing blank, small and large DSDs were $21-37 \mathrm{~Pa} \cdot \mathrm{s}, 154-264 \mathrm{~Pa} \cdot \mathrm{s}$, and $110-214 \mathrm{~Pa} \cdot \mathrm{s}$, respectively. At high shear, the apparent viscosity ranges for all systems was $2-5 \mathrm{~Pa} \cdot \mathrm{s}$ and were statistically similar. Consistency (a) of both large and small GMO DSDs increased slightly over time whereas b values concurrently decreased. The small DSD GMO emulsions saw their $a$ values increase from $141 \mathrm{~Pa} \cdot \mathrm{s}$ on Day 0 to $248 \mathrm{~Pa} \cdot \mathrm{s}$ on Day 28 whereas $b$ values decreased from 0.3 to 0.1 . The large GMO DSDs exhibited similar trends, with consistency increasing from 98 to $254 \mathrm{~Pa} \cdot \mathrm{s}$ and $\mathrm{b}$ values decreasing from 0.3 to 0.1 . Finally, the GMO control dramatically increased in $a$ from Day 0 to Day 7 from 20 to $269 \mathrm{~Pa} \cdot \mathrm{s}$ and remained higher than the $a$ values of both GMO emulsions during storage. On Day 0 , its $b$ value was 0.5 but decreased to $<0$ by day 7 , below that of the emulsions $(\sim 0)$. The consistency index of all GMO-based systems followed the same trend seen in the reinforcement results, indicating that there was some degree of structural change occurring with time, however slowly.

Overall, since the $b$ values obtained by fitting the viscosity to a power law equation were uncharacteristically negative in spite of favourable $\mathrm{R}^{2}$ values. This indicates that the fitting provides a summary of the observed trends (i.e. increases in viscosity) with the calculated $a$ values, the $b$ value trends, or lack thereof, are not reliable and these emulsions do not adhere to power law fluid behaviour under shear. 


\subsubsection{Particle size and network interactions}

Figure 3.6 shows a general summary of interactions between particles of different size distributions within a fat crystal network and the associated changes in rheological behaviour. Particle surfaces can differ in their interactions with the surrounding fat crystal network (shown in black), which can reinforce the network (Rafanan and Rousseau, 2017). Strong droplet-droplet interactions (shown in red) can also dramatically reinforce the network. Emulsifiers at the droplet interface can promote greater interaction strength with the surrounding fat crystal network through interfacial crystallization and sintering. Emulsifiers with a disordered, branched structure keeps adjacent droplets sterically separated, allowing droplets to interact only weakly (i.e., PGPR). Droplet size also influences the overall number of interactions between particles available in a system, which can influence whether the droplet network is rigid (i.e., MAGs) or more mobile (i.e., PGPR). The current study showed that increasing reinforcement and viscosity is best achieved in stronglyinteracting systems by reducing droplet size (Figures 3.3B, 3.4B, and 3.5B). Conversely, for weakly-interacting droplets in this HSO network, reinforcement and viscosity increased by increasing droplet size (Figures 3.3A, 3.4A, and 3.5A). Previous studies have shown that networks consisting of weakly-interacting small particles display higher viscosities compared to coarser particle suspensions. However, the addition of particles to an existing particle network can have the opposite effect (Do et al., 2007; Farris, 1968; Genovese, 2012). This is ultimately due to small particles widening the overall particle size distribution (i.e., filler and network particles) and lubricating the movement of large network particles. Addition of filler particles closer in size to those of the network simply contribute solid matter to the suspension which impede shear flow (Farris, 1968). Although DSDs may be significantly different, as was the case with GMO, droplet 
packing within network voids must be sufficiently different to invoke any difference in reinforcement and viscosity.

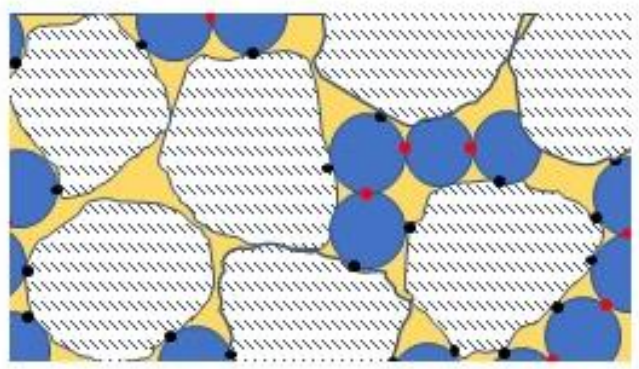

Large DSD-strong filler network

- Strong reinforcement

- Significant viscosity increase

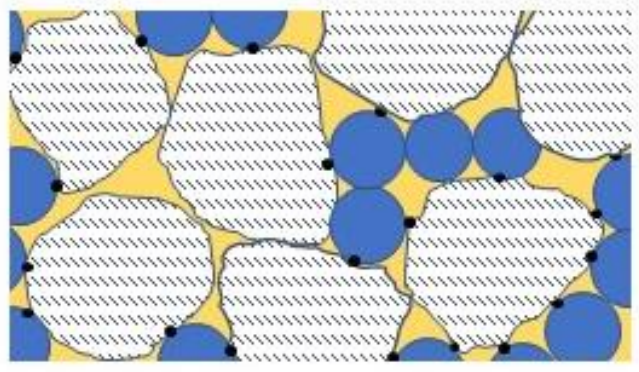

Large DSD-weak filler network

- Moderate reinforcement

- Moderate viscosity increase

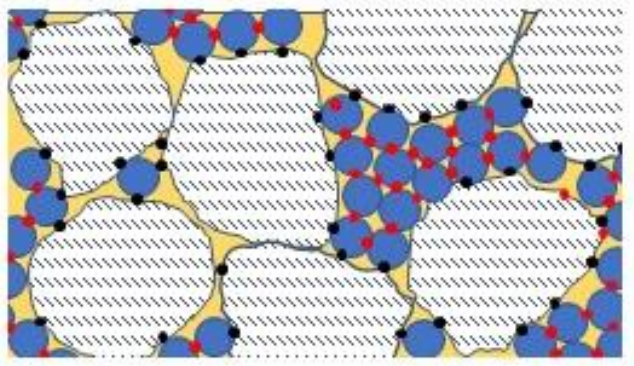

Small DSD-strong filler network

- Most reinforcement

- Highest viscosity increase

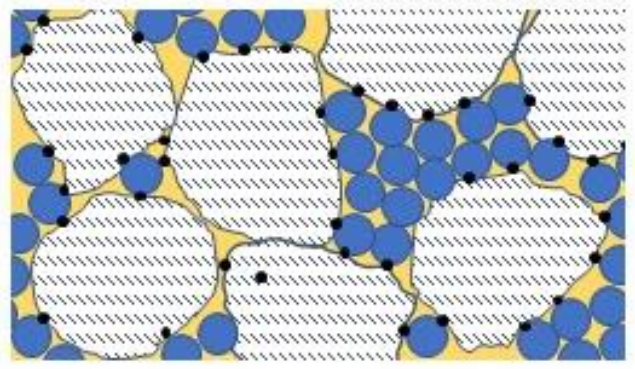

Small DSD-weak filler network

- Minor reinforcement

- Minor viscosity increase

Figure 3.6 - Schematic summary of the role droplet size, and particle interaction strength on fat crystal network rheology. Red spheres between droplets vary in strength depending on interfacial characteristics (emulsifier structure, interfacial crystallization etc.). Black spheres are contact points between droplets and the surrounding fat crystal network. 


\subsection{Conclusion}

This study has illustrated how the introduction of emulsion droplets within a fat crystal network imparts significant changes on the rheology of the resulting composite. The present study has shown that not only are emulsifier-dependent interfacial characteristics factors important in determining the extent of these changes, but also the particle size/volume fraction. The droplet interface, which affects particle interactions with their nearest neighbours, works in concert with the droplet packing behaviour to alter the rheology of fat crystal-stabilized emulsions. It is shown here that filler-filler interactions leading to aggregation enhance the rigidity of fat crystal networks. By categorizing droplets as strongly (i.e., MAGs) or weakly-interacting (i.e., PGPR), and then optimizing droplet size distribution, it is possible to more systematically produce fat crystalstabilized emulsions with desired rheological properties. Further research on fat crystal network stabilized emulsions can expand on this research to include whether aggregates of small droplets on the same size scale as larger single droplets have similar rheological behaviour. This would better elucidate the viscoelastic nature of droplet aggregates and explore the reinforcing potential of aggregates in fat crystal network. This approach may be used as a tool to tailor texture in relevant industrial applications (foods, pharmaceuticals, cosmetics, etc.). 


\title{
Chapter 4 - Effect of shear and interfacial fat crystallization on release of water-soluble dye from water-in-oil emulsions
}

Published in LWT - Food Science and Technology (Rafanan and Rousseau, 2019b)

\begin{abstract}
The microstructure and associated dye release behaviour of water-in-oil (W/O) emulsions stabilized with either a liquid or solid-state interfacial layer were studied under static and shear conditions. The emulsions contained high-melting fat crystals in the oil continuous phase which provided network stabilization. Two surfactant types were used to generate and stabilize the emulsions: Polyglycerol polyricinoleate (PGPR), a liquid-state surfactant at room temperature, and monoacylglycerols glycerol monostearate (GMS) and glycerol monooleate (GMO) which are solid at the same temperature. The latter two surfactants resulted in droplets exhibiting interfacial crystallization, whereas only network stabilization was present in the PGPR-based emulsion (i.e., no interfacial crystallization). Release of Rhodamine B, a water-soluble dye, from the dispersed aqueous phase of the W/O emulsions into an agar gel was quantified using confocal microscopy. Emulsions stabilized with liquid surfactant displayed greater dye release compared to the GMS and GMO emulsions. The PGPR emulsions also showed higher shear-mediated dye release than under quiescent conditions, whereas no such differences were seen with the GMS and GMO emulsions. Ultimately, the interfacial structure of the dispersed aqueous droplets was the dominant factor controlling the release of water-soluble dye from these W/O emulsions.
\end{abstract}

Keywords: fat crystal network, emulsion, encapsulation, controlled release, shear stability 


\subsection{Introduction}

Water-in-oil (W/O) emulsions are dispersions present in goods such as foods, cosmetics and personal products. The stability of the dispersed aqueous phase in such systems is most often attained using small-molecule surfactants or solid particles. In food-based W/O emulsions, small fat crystals present at the interface have been shown to act as an effective physical barrier against physical breakdown (Frasch-Melnik et al., 2010; Nadin et al., 2014).

A growing area of interest is the use of solids-stabilized W/O emulsions for encapsulation and release of aqueous compounds sensitive to light, air or other compounds (Melle et al., 2005), as these have shown improved encapsulation potential over surfactant-stabilized emulsion droplets (Frasch-Melnik et al., 2010; Frelichowska et al., 2009; Nadin et al., 2014). While recent efforts have concentrated largely on novel, food-safe particles suitable for stabilizing the interface or to extend shelf stability under static conditions, little has been reported on their stability and release behaviour under shear. This is particularly relevant as typical experimental conditions do not reflect actual material behaviour during consumer use, such as spreading or mixing. Furthermore, few methods are available to quantify the release of encapsulated compounds from emulsions into a release medium during shear.

Emulsions with crystalline fat at the interface have been used to encapsulate salt within the dispersed aqueous phase of W/O emulsions (Frasch-Melnik et al., 2010; Nadin et al., 2014). Nadin et al. found that glycerol monostearate (GMS)-stabilized droplets were more effective for retaining salt than polyglycerol polyricinoleate (PGPR) as the former resulted in a solid interfacial layer (Nadin et al., 2014). Release was triggered by an increase in temperature, which melted the fat at the interface, promoting droplet destabilization and subsequent release of encapsulated cargo. 
Emulsion stability and interfacial behaviour will significantly impact the release of encapsulated compounds through different barriers. For example, transport across a membrane (e.g., through skin) can be enhanced, or delayed depending on the type of stabilizer present at the interface of emulsion droplets. In a study on the skin absorption of retinol encapsulated within oil-in-water $(\mathrm{O} / \mathrm{W})$ emulsions, Frelichowska et al. showed that retinol delivered via Pickering-stabilized droplets accumulated primarily within the stratum corneum, the upper-most layer of the dermis, while small-molecule surfactants facilitated greater retinol deposition into the dermis layer (Frelichowska et al., 2009). In another study on encapsulated retinol, Ghouchi Eskandar et al. reported that once emulsion droplets had destabilized within the epidermis, the Pickering particles originally at the droplet surface interacted with the compound of interest and moved further into the skin layer (Ghouchi Eskandar et al., 2009). Sarmento et al. found that an oral insulin preparation encapsulated within a solid lipid shell offered protection from the harsh environment of the stomach and could be absorbed in the small intestine (Sarmento et al., 2007). In foods, the majority of release studies have focused on O/W emulsions. In a study performed by Marefati $e t$ al., modified starch used as an interfacial stabilizer effectively encapsulated curcumin during in vitro enzymatic digestion tests (Marefati et al., 2017). Matos et al. found that modified starchstabilized droplets encapsulated resveratrol twice as effectively as those stabilized by a non-ionic surfactant (polysorbate 20), and slowed release of the encapsulated cargo effectively under static release conditions (Matos et al., 2018). While the studies of Matos et al. and Marefati et al. showed the potential for effective encapsulation, these were performed under static conditions and did not account for shear effects such as mastication or spreading.

Determining how the interface contributes to encapsulation efficiency with regards to applied shear is problematic for a few reasons. Established methods such as the Franz cell require the use 
of a permeable membrane (i.e., excised skin), specialized equipment and static exposure conditions to determine the absorption of a compound of interest through the membrane. As well, this method requires large volumes of release medium and high loading for quantification. The present method repurposes more common lab equipment (controlled strain rheometer and confocal microscope) to quantify the release of a water-soluble fluorescent dye from a fat-crystal stabilized emulsion into a neighbouring hydrogel. This method uses only a fraction of the release medium described by previous static-only methods (Frasch-Melnik et al., 2010; Nadin et al., 2014). As well, this method captures the release of a dye within 5 min of exposure; far less than typical diffusion tests that can take hours to days to complete.

The release behaviour via confocal microscopy herein explored relied on Rhodamine B, dye commonly used as a fluorochrome. Its quantification has been explored in highly diverse fields, e.g., using remote aerial fluorescence sensing to determine diffusion processes and local concentrations along shores over time (Clark et al., 2014). Confocal microscopy has also been used to determine the relative fluorescence intensity of fluorescent-tagged structures and penetration depth of dyes in skin tissue (Alvarez-Román et al., 2004; van Kuijk-Meuwissen et al., 1998). It presents a convenient way to quantify rhodamine diffusion through a medium while controlling variables such as laser light intensity, field of view and objective working distance.

The purpose of this study was three-pronged: i) develop a novel confocal microscopy method to detect and quantify the release of a water-soluble fluorescent dye from within W/O emulsions; ii) compare dye release from emulsion droplets under quiescent and shear conditions; and iii) contrast the resistance to release of $\mathrm{W} / \mathrm{O}$ emulsions generated using a liquid-state emulsifier vs. those that are higher-melting and likely to crystallize at the oil-water interface. 


\subsection{Materials and methods}

\subsubsection{Emulsion construction}

Each emulsion consisted of $20 \mathrm{~g}$ aqueous and $80 \mathrm{~g}$ oil fractions per $100 \mathrm{~g}$ emulsion. Hydrogenated soy oil (acid value $<0.1 \%$ ) (HSO, Bunge international, Mississauga, ON, Canada) and canola oil (acid value $<0.1 \%$ ) purchased at a local supermarket comprised the oil phase common to all emulsions used in the study. The monoacylglycerols (MAGs) glycerol monooleate (GMO, 90\% monoglycerides, Monomuls O18, BASF) and glycerol monostearate (GMS, 95\% monoglycerides, Alphadim SBK, Corbion, Mississauga, ON, Canada) were surfactants that promoted interfacial crystallization whereas PGPR (Nealanders, Mississauga, ON, Canada) did not promote crystallization at the interface at room temperature (Rafanan and Rousseau, 2019, 2017). Emulsion compositions are given in Table 1 . The aqueous phase consisted of $50 \mathrm{mmol} / \mathrm{L} \mathrm{NaCl}$ and 0.2 mmol/L Rhodamine B (Acros Organics, Thermo Fisher Scientific, Geel, Belgium) dissolved in deionized water. The presence of $\mathrm{NaCl}$ in the aqueous phase was required to prevent double emulsion formation during emulsification (Pradhan and Rousseau, 2012).

Table 4.1: Emulsion compositions on wt $\%$ basis. The aqueous phase consisted of $0.5 \mathrm{M} \mathrm{NaCl}$ and $0.2 \mathrm{mmol} / \mathrm{L}$ Rhodamine B dye.

\begin{tabular}{c|ccc}
\hline Component & PGPR & GMS & GMO \\
\hline Oil Phase & & & \\
Surfactant & 0.4 & 3.2 & 1.6 \\
HSO (hard fat) & 14.4 & 14.4 & 14.4 \\
Canola oil & 65.2 & 62.4 & 64 \\
\hline $\begin{array}{c}\text { Aqueous Phase } \\
\text { Water }\end{array}$ & 20 & 20 & 20 \\
\hline
\end{tabular}




\subsubsection{MAG-stabilized emulsions (interfacial crystallization)}

To ensure narrow droplet size distributions and efficient mixing, a two-stage protocol consisting of coarse and fine emulsification was used. The oil phase constituents were heated while mixing with a magnetic stir bar at $150 \mathrm{rpm}$ at $85^{\circ} \mathrm{C}$ for $10 \mathrm{~min}$. The room temperature aqueous phase was then added dropwise to the molten oil phase. The aqueous phase was added slowly to prevent the temperature from dipping below the crystallization temperature. Once the aqueous phase was completely added, the coarse emulsion was mixed at $200 \mathrm{rpm}$ for $10 \mathrm{~min}$ at $85{ }^{\circ} \mathrm{C}$. Coarse emulsions were then mixed via rotor-stator at $7000 \mathrm{rpm}$ for $3 \mathrm{~min}$. The GMS-stabilized emulsions were homogenized via high-pressure homogenization ( $1^{\text {st }}$ stage: $6.9 \mathrm{MPa}, 2^{\text {nd }}$ stage: $48.2 \mathrm{MPa}$ ) continuously at $85^{\circ} \mathrm{C}$ for $5 \mathrm{~min}$. The GMO-stabilized emulsions were similarly homogenized at pressures of 3.4 and $6.9 \mathrm{MPa}$ for the $1^{\text {st }}$ and $2^{\text {nd }}$ stages, respectively. Once homogenized, the emulsions underwent shear cooling to form the crystal network and interfacial crystallization. Both GMO- and GMS-stabilized emulsions were poured into a metal beaker partially submerged in an ice bath and mixed continuously via impeller at $260 \mathrm{rpm}$ to $13{ }^{\circ} \mathrm{C}$. The GMS concentration was at the CMC of $4 \mathrm{wt} \%$ of the oil phase, while GMO was below the CMC (Ghosh and Rousseau, 2009; Ghosh et al., 2011). Above 2 wt\% oil phase using the present emulsification conditions, droplet sizes were significantly smaller, and the fat crystal network structure was not comparable to the GMS and PGPR-stabilized emulsions.

\subsubsection{PGPR-stabilized emulsions (liquid interface)}

The fat crystal network was formed prior to emulsification as crystallizing after emulsification promoted smaller droplet size distributions that were not comparable to the MAG-stabilized emulsions (i.e., no overlap in size distribution). The HSO and canola oil were heated to $85{ }^{\circ} \mathrm{C}$ and mixed thoroughly via magnetic stirrer at $150 \mathrm{rpm}$. The hot oil + fat mixture was then shear cooled 
at $262 \mathrm{rpm}$ via impeller to $13{ }^{\circ} \mathrm{C}$ in the ice bath-cooled metal beaker as described in the previous section. The PGPR was added to the cool oil + fat blend and mixed via impeller at $260 \mathrm{rpm}$. The aqueous phase was added in a steady stream while shearing at $50 \mathrm{rpm}$ for $3 \mathrm{~min}$ once the aqueous portion was added completely. The emulsion was then transferred to storage containers and aged for $24 \mathrm{~h}$.

\subsubsection{Emulsion characterization}

\subsubsection{Droplet size and solid fat content}

Dispersed droplet size and solid fat content (SFC) were determined using a Bruker mq20 pulsed field gradient NMR (Bruker Canada, Milton, ON, Canada). Immediately after preparation, the control and emulsion samples were pipetted into NMR tubes to heights of $1 \mathrm{~cm}$ and $6 \mathrm{~cm}$, respectively. The SFC of the emulsions was determined at $4{ }^{\circ} \mathrm{C}$ using the application sfc_lfc v2.51 whereas the droplet d50 values were determined using the water droplet v2.51 application. When droplet sizes were below the threshold for NMR determination of droplet size $(\sim 1 \mu \mathrm{m})$, image analysis was used as described below.

\subsubsection{Melting point and enthalpy}

Solid fat temperature and enthalpy of melting were determined by differential scanning calorimetry (DSC) on a Q2000 DSC unit (TA instruments, New Castle DE, USA). To do so, 10$15 \mathrm{mg}$ aliquots of each emulsion were hermetically sealed within aluminum pans and heated from 5 to $90{ }^{\circ} \mathrm{C}$ at $5{ }^{\circ} \mathrm{C} / \mathrm{min}$ after equilibration at $5^{\circ} \mathrm{C}$ for $1 \mathrm{~min}$. Peak melting temperatures and corresponding enthalpies were recorded using the DSC's built-in software (TA Universal Analysis 2000, Version 4.5, Waters LLC, Milford, MA, USA). 


\subsubsection{Rheology}

Emulsion viscosity was measured using an Anton-Paar MCR302 (Anton-Paar, Graz Austria) rheometer with a Peltier-controlled, Teflon-coated flat stage (PPTD200) and 25mm parallel plate measuring system geometry (PP25/TL). Apparent viscosity at increasing shear rates was measured from 1 to $100 \mathrm{~s}^{-1}$ with the RheoPlus software (v3.40). Emulsions were applied to the stage surface and compressed with the parallel plate to a gap of $1.0 \mathrm{~mm}$ and measured at $25{ }^{\circ} \mathrm{C}$. Apparent viscosity at $1.6 \mathrm{~s}^{-1}$ was recorded as this was the shear rate applied to the emulsions during the release test under shearing conditions.

\subsubsection{Microscopy}

Emulsion morphology (dispersed phase and fat/surfactant crystals) was assessed using a Zeiss Axiovert 200M inverted light microscope with a $63 \mathrm{X}$ objective and 10X ocular (630X magnification, Zeiss Canada, Toronto, ON, Canada) at room temperature. Brightfield and polarized light microscopy were performed on each emulsion after crystallization. A small amount of emulsion sample was diluted with canola oil prior to sealing the sample with a cover slip. Dilution of emulsions with canola oil was necessary to achieve a thinner sample layer to isolate individual droplets for clear imaging of the droplet oil-water interface. Images captured on QImaging CCD camera were analyzed using qCapture Pro software v7.0 (QImaging, Surrey BC, Canada).

\subsubsection{Static and shear-mediated diffusion}

The release medium consisted of $1.5 \mathrm{wt} \%$ agar (powder, Setexam, Kenitra, Morocco) in DI water. Solid agar was solubilized for 15 min prior to boiling. Once boiled and fully dissolved, the hot agar solution was poured into a clean, empty glass Petri dish to a depth of $1 \mathrm{~cm}$ and aged overnight 
before the emulsion was applied to the surface of the releasing medium. The resulting agar gels had a smooth surface.

The rheometer described in the previous rheology section was used to study dye release behaviour as per the set-up in Figure 1A. The agar served as the release medium into which Rhodamine B was to be released. The agar was placed on the center of the rheometer's bottom plate and brought to $25^{\circ} \mathrm{C}$. The upper surface of the agar then behaved as the bottom plate of the rheometer and the zero gap was set. One-day old emulsions were spread onto the agar surface, ensuring that no air pockets were present between the emulsion and agar surface. The parallel plate geometry was lowered onto the sample to a gap height of $1 \mathrm{~mm}$. Excess emulsion was trimmed gently as to not break the agar gel beneath. For static dye release, the trimmed emulsion was permitted to sit between the release medium and the parallel plate for 5 min without added shear. The trimmed dye-loaded emulsions were then rotationally sheared at $1.6 \mathrm{~s}^{-1}$ for $5 \mathrm{~min}$. The shear applied mimicked conditions akin to spreading a sample topically (i.e., a cream or ointment) under gentle application conditions (Brummer and Godersky, 1999), and was sufficiently gentle to avoid deteriorating the underlying agar gel. This shear rate provided enough force for each emulsion to yield and exhibit viscous behaviour. After 5 min exposure, the parallel plate was lifted, and excess emulsion left on the surface was gently removed with a stainless-steel flat spatula. 


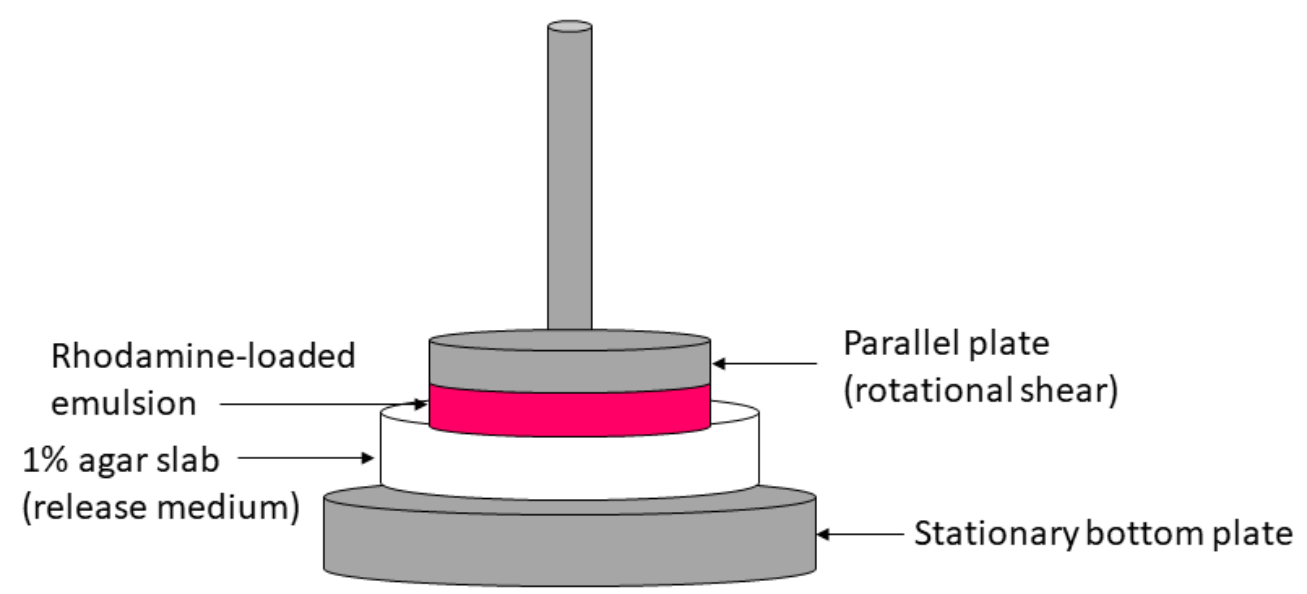

B
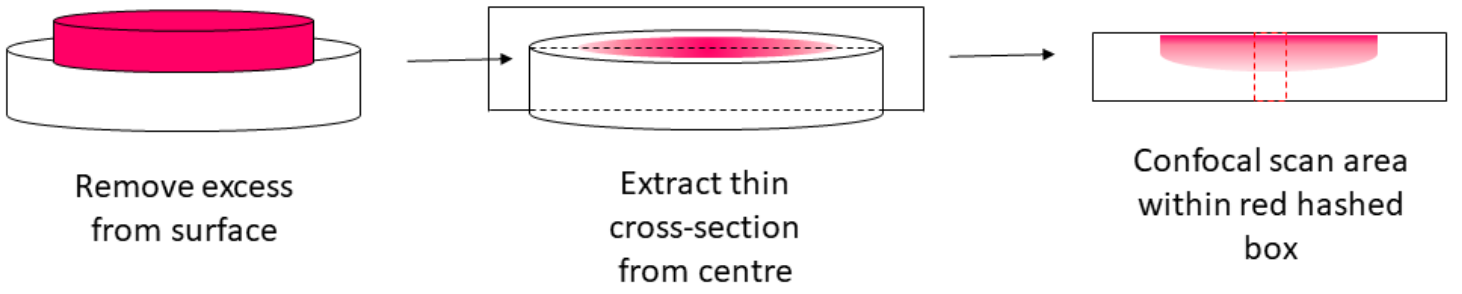

Figure 4.1: Schematic of Rhodamine B release set-up and microscopy preparation. (A) shows how Rhodamine B is introduced to the agar slab. The dye-loaded emulsion is applied to the top of the agar slab, compressed to a gap thickness of $1 \mathrm{~mm}$ and trimmed. The emulsion is sheared by the rotating parallel plate while the agar slab and bottom plate remain stationary. (B) shows the process of preparing and analyzing the agar slab after exposure. Excess emulsion is cleaned from the surface and a medial cross section is removed along the diameter. The area bound by the red dashed box is the confocal scan area of $5000 \times 510$ pixels (y-axis x x-axis). 
A $2 \mathrm{~mm}$ cross-section was then cut through the circular slab (Figure 1B). The cut edge was placed on a glass slide and covered with a coverslip. The slab was then imaged immediately by confocal microscopy to limit further dye diffusion through the gel.

\subsubsection{Confocal imaging and analysis}

Confocal imaging was performed to quantify the passage of fluorescent rhodamine through the agar slab using a Zeiss LSM800 (Carl Zeiss Canada Ltd, Toronto, ON, Canada) using a A-plan 10x/0.25 objective. Pinhole size was $47 \mu \mathrm{m}$ (Airy unit $=1$ ). Excitation was provided by $\mathrm{HeNe} 561$ $\mathrm{nm}$ laser at an intensity of $0.37 \%$. Rhodamine B emission was measured using a long pass filter with a cut-off of $562 \mathrm{~nm}$. The scan area of each image was 510 x 5000 pixels, referred to as the $\mathrm{x}$ - and $\mathrm{y}$-axis, respectively. Images were captured at 5 pixels/sec using Zen Blue software. Each RGB confocal image was converted to 2D intensity matrices using Matlab (version 2016b, Mathworks Inc, Natick, MA, USA). The red channel of the resulting matrices was used for image analysis. Each matrix element consisted of a value between 0 and 255, corresponding to pixel intensity. Dye-containing regions had intensities of 20 or greater since values below 20 were too low to distinguish from noise. A moving average of 5 was taken at each pixel location (i.e., average of the bordering intensity values) to smooth the intensity data.

The depth of dye migration through the agar during exposure is herein referred to as "path length". Each confocal image scan area corresponded to that bounded by the red dashed box shown in Figure 4.1B(far right). Figure 4.2 shows an exemplar confocal image that captures the agar surface, and the migration of dye through the gel along the y-axis. The gel surface of each image was mathematically determined by calculating the greatest rate of change in intensity moving down

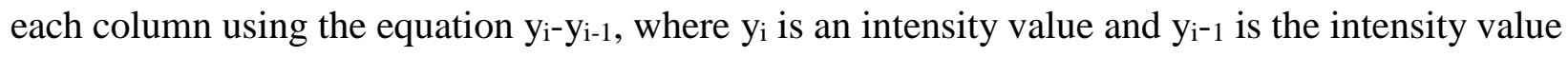
directly preceding it. This resulted in a matrix of values corresponding to changes in intensity 
between adjacent pixels as one moves down a single matrix column. The gel surface was identified as the region where the change in intensity was greatest. The average intensity values as a function of distance from the surface are shown in Appendix Figure 4.1. Dye diffusion through the gel travelled downward through the gel and appeared as a gradient along the y-axis of each image. The pixel location at which the signal decreased to below the threshold value of 20 was taken as the end of the moving dye front (Dotted horizonal line, - in Appendix Figure 4.1). 
A

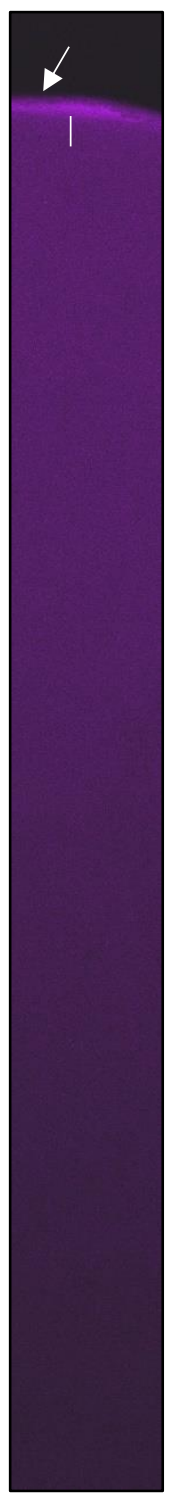

B

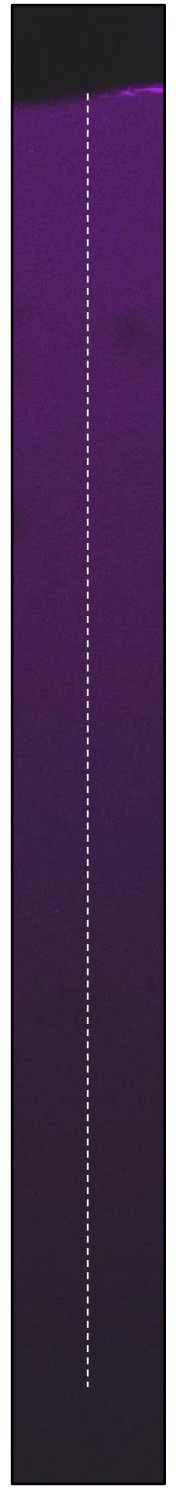

Figure 4.2 Confocal image of typical gel cross-sections after dye was applied to the surface. The images here correspond to the red dashed box in Fig. 1B. A - confocal image showing the int +50 region corresponding to the area of highest local dye concentration (solid white line) within the gel after exposure; B - dye path length (dashed line). Bright areas at the gel surface (arrow) are residual emulsion remaining after dye exposure. The average pixel intensities are measured 50 pixels from the surface (along solid white line). 
The area close to the surface of the gel contained the highest local concentration of Rhodamine B. This area, referred to as "Int +50 " began at the dashed vertical line in Appendix figure 4.1. The intensity was converted to concentration via calibration curve. The int +50 is the average Rhodamine concentration of pixels 50 to 100 from the gel surface and this was calculated for each of the $510 \mathrm{x}$-axis values for each image.

\subsection{Statistical analysis}

One-way and two-way ANOVAs were performed on path length and int+50 data using SigmaPlot 11 (Systat Software Inc., San Jose, CA, USA). Triplicate experiments and measurements were taken for all treatments $(n=3)$.

\subsection{Results and Discussion}

\subsubsection{Emulsion texture and structure}

Figure 4.3 shows the consistency of each emulsion after extrusion from a syringe to show how well each emulsion maintained its structure. Each emulsion yielded under the force of extrusion. The PGPR-stabilized emulsion exhibited a viscous fluid consistency and coated the inner walls of the vessel before settling at the bottom of the container and held no structure after extrusion. By contrast, the GMO and GMS-stabilized emulsions were both soft solids. The GMS-based emulsion exhibited more solid-like character than its GMO-based counterpart, as evidenced by the superior shape retention after extrusion. 

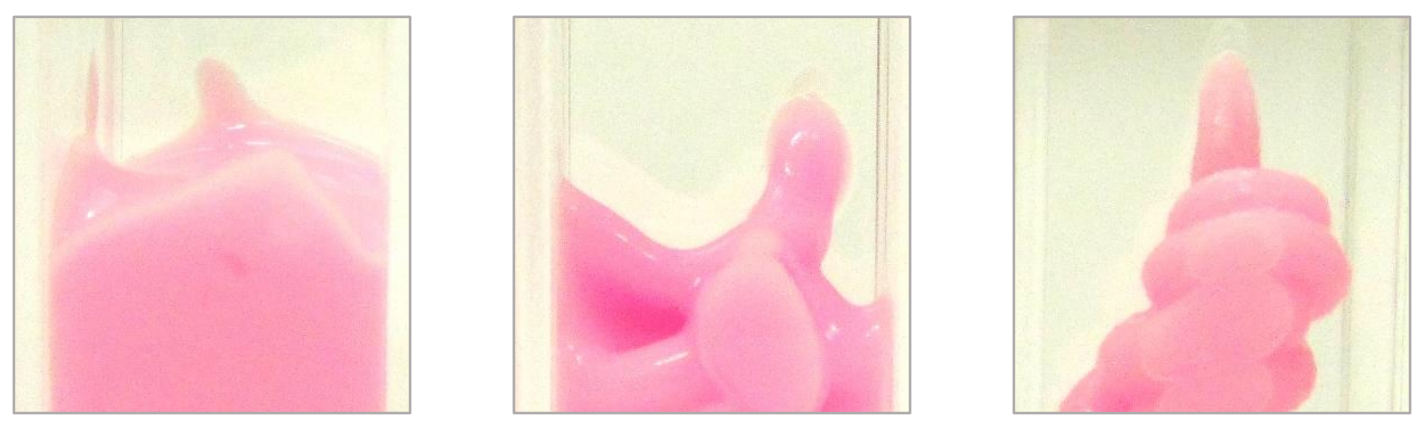

Figure 4.3 Comparison of macroscopic texture W/O emulsions stabilized by PGPR (left), GMO (middle), and GMS (right). Each emulsion was extruded into a square plastic cuvette, allowed to settle for $1 \mathrm{~min}$ and photographed.

Each emulsion contained $14 \mathrm{~g} \mathrm{HSO} / 100 \mathrm{~g}$ oil phase. However, as the MAGs crystallized, the SFC of the GMO and GMS-based emulsions were higher than the PGPR sample (Table 2). This may have contributed, in part, to the difference in texture seen in Figure 4.3. Droplet size distributions were matched as closely as possible with overlapping droplet sizes (Figure 4.4 and Table 4.2). Although the d50 values of the GMO emulsions were lower than PGPR and GMS, the size distributions of all emulsions overlapped. Each emulsion showed fluid-like behaviour under the test shear conditions (Table 4.2), with the PGPR and GMO emulsions showing viscosity values of $\sim 28$ and $\sim 62 \mathrm{~Pa} \cdot \mathrm{s}$, respectively. The GMS-based emulsion had significantly higher viscosity $(\sim$ $174 \mathrm{~Pa} \cdot \mathrm{s})(\mathrm{p}<0.05)$. DSC showed that the melting points of all emulsions were similar at $55-57$ ${ }^{\circ} \mathrm{C}$ (Table 4.2 and Appendix figure 4.2), though the melting enthalpies were significantly different (PGPR: $164 \pm 7$, GMS: $228 \pm$ 10, GMO: $128 \pm 5 \mathrm{~J} / \mathrm{g})(\mathrm{p}<0.05)$, and likely resulted from differences in SFC (Rafanan and Rousseau, 2019a). 


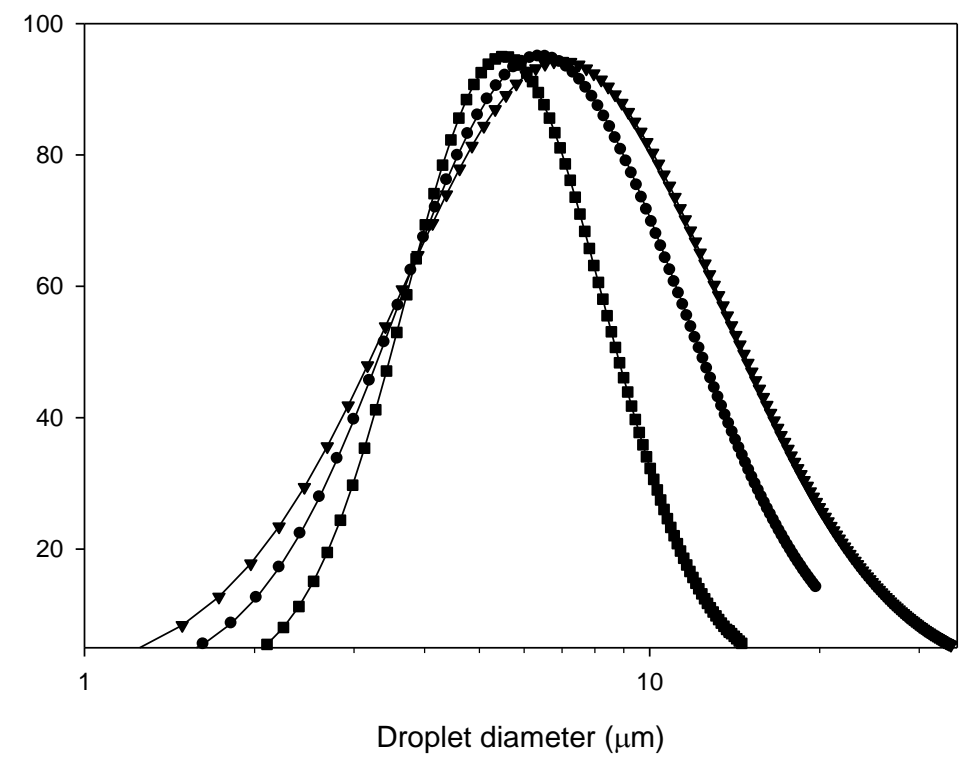

Figure 4.4 Droplet size distributions of fat crystal-stabilized emulsions using the following emulsifiers: $\nabla$ - PGPR $(\sim 9 \mu \mathrm{m})$, $\mathbf{-}$ - GMO $(\sim 5 \mu \mathrm{m})$, and $\bullet$ - GMS $(\sim 10 \mu \mathrm{m})$. Values in brackets identify average number-weighted droplet size $(n=3)$.

Table 4.2: Physical characteristics of fat crystal network-stabilized emulsions. Droplet diameter (d50), SFC, apparent viscosity at $1.6 \mathrm{~s}^{-1}$, and DSC heating data (melting point and enthalpy, $\Delta \mathrm{H}$ ) of the PGPR-, GMS- and GMO-stabilized emulsions. Statistical differences $(\mathrm{p}<0.05)$ are given by an asterisk *.

\begin{tabular}{cccc}
\hline Parameter & PGPR & GMS & GMO \\
\hline Droplet size & $9.1 \pm 1.3$ & $10.4 \pm 2.7$ & $5.4 \pm 1.0^{*}$ \\
$(\mathrm{D} 50, \mu \mathrm{m})$ & $15.4 \pm 0.1$ & $18.3 \pm 0.4^{*}$ & $14.7 \pm 0.1$ \\
SFC $(\%)$ & $28.2 \pm 19.6$ & $173.6 \pm 26.5^{*}$ & $62.4 \pm 20.6$ \\
Apparent viscosity at & $56 \pm 0$ & $55 \pm 0$ & $57 \pm 0$ \\
$1.6 \mathrm{~s}^{-1}(\mathrm{~Pa} \cdot \mathrm{s})$ & $165 \pm 7^{*}$ & $228 \pm 10^{*}$ & $128 \pm 5^{*}$ \\
Melting point $\left({ }^{\circ} \mathrm{C}\right)$ & & \\
$\Delta \mathrm{H}(\mathrm{J} / \mathrm{g})$ & &
\end{tabular}

Representative brightfield microscopy images of freshly-prepared emulsions (Figure 4.5 top row) showed similar droplet sizes as reported through pulsed NMR. Via PLM, there was no evident 
interfacial crystallization for the PGPR-stabilized droplets (Fig. 5D). The MAG-stabilized emulsions (GMS and GMO, Figures 4.5E and 4.5F, respectively) displayed crystalline layers, as per the inset image of each figure. The similar fat crystal network for all emulsions confirmed that the major structural difference between the emulsions was the droplet interface. Post-shear imaging showed that the PGPR-stabilized emulsion droplets remained devoid of interfacial crystals (Figures 4.6A and 4.6D) nor were there any significant changes to the emulsion appearance after shear. Expectedly, both MAG emulsions maintained the crystalline shell surrounding the droplets (GMS: Figures 4.6B and 4.6E, GMO: Figures 4.6C and 4.6F).
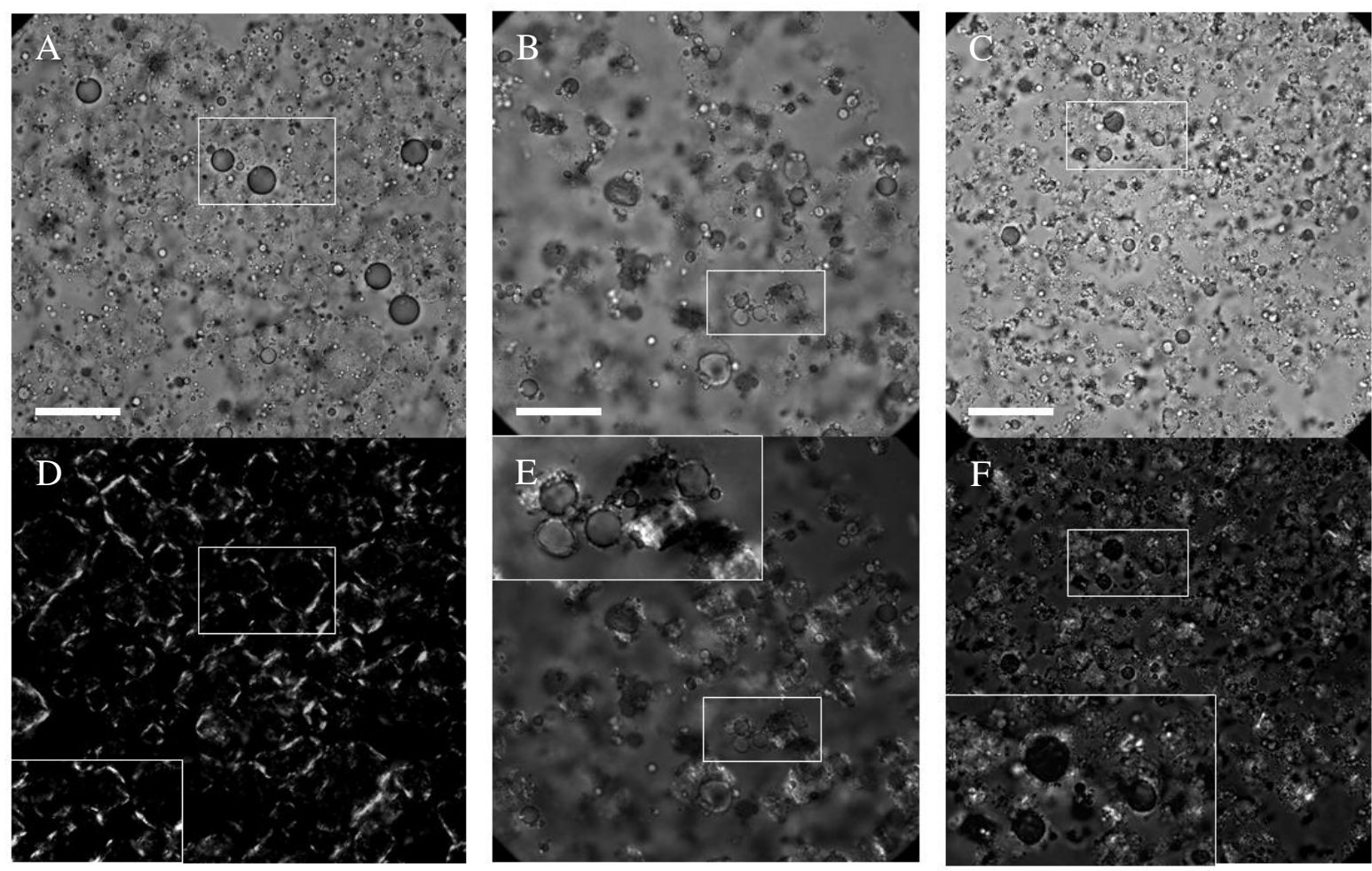

Figure 4.5 Microscopic morphology of emulsions stabilized by different emulsifiers. Left to right: PGPR, GMS, and GMO. Top row images (A, B, C) are taken under brightfield imaging. Bottom row images $(\mathrm{D}, \mathrm{E}, \mathrm{F})$ are the same field of view under polarized light. Inset images on images $\mathrm{D}$, $\mathrm{E}$, and $\mathrm{F}$ are zoomed in on the white boxed region in the images above. Scale bar on all brightfield images is $50 \mu \mathrm{m}$. 

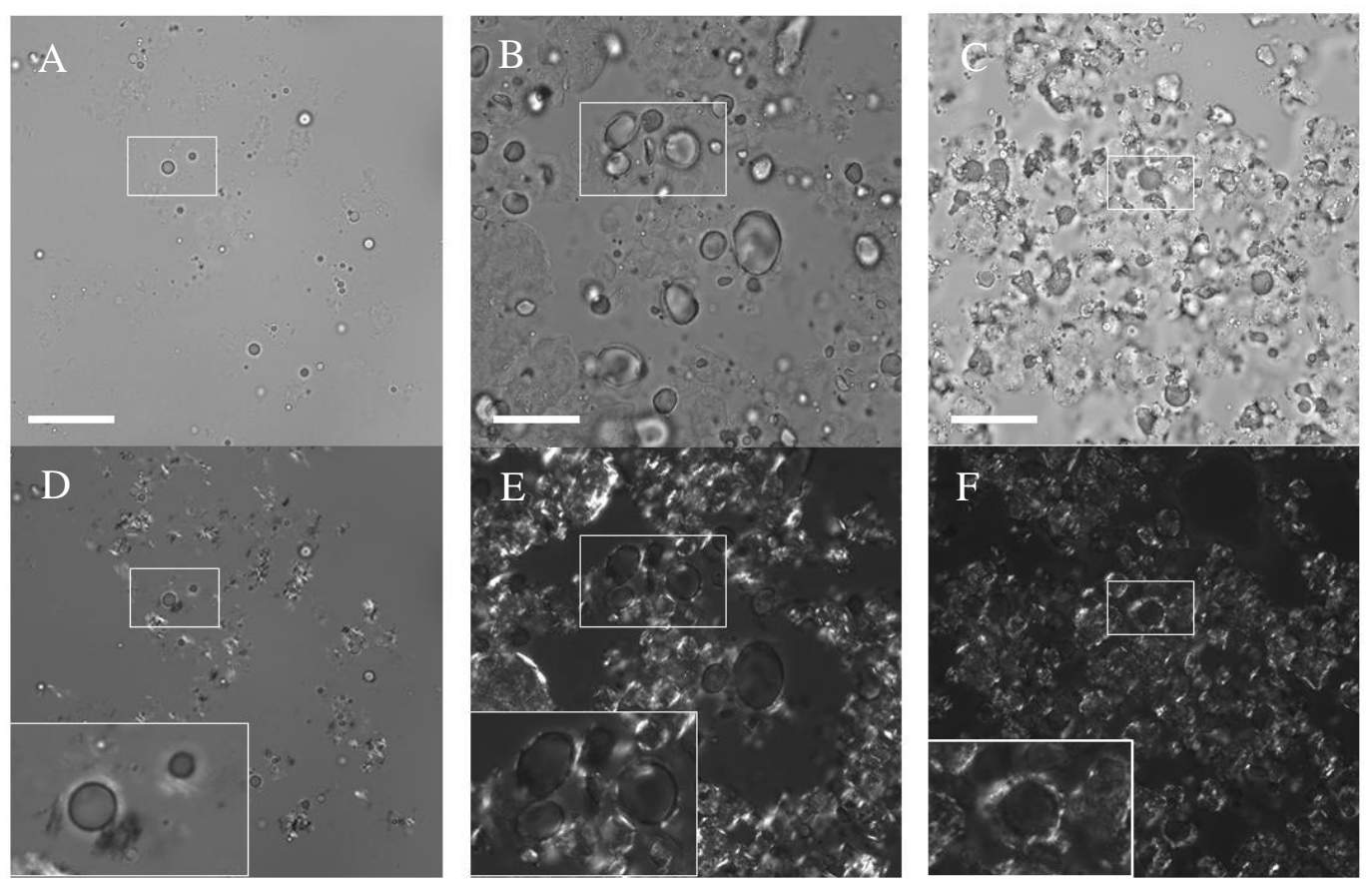

Figure 4.6 Microscopic morphology of emulsions after shear treatment. Left to right PGPR, GMS, and GMO. Top row images $(\mathrm{A}, \mathrm{B}, \mathrm{C})$ are taken under brightfield imaging. Bottom row images $(\mathrm{D}, \mathrm{E}, \mathrm{F})$ are the same field of view under polarized light. Inset images on images $\mathrm{D}, \mathrm{E}$, and $\mathrm{F}$ are zoomed in on the white boxed region on the images above. Scale bar on all brightfield images is $50 \mu \mathrm{m}$.

\subsubsection{Confocal image analysis}

The PGPR-stabilized droplets exhibited a higher average path length under shear $(708 \pm 78 \mu \mathrm{m})$ and static conditions $(647 \pm 120 \mu \mathrm{m})$ than the MAG-based emulsions $(\mathrm{p}<0.05)$ (Figure 4.7). Under shear, the GMS and GMO path lengths were $517 \pm 50 \mu \mathrm{m}$ and $513 \pm 9 \mu \mathrm{m}$, respectively, and 548 $\pm 80 \mu \mathrm{m}$ and $477 \pm 5 \mu \mathrm{m}$ under static conditions, respectively. There was no statistical difference in path length with respect to shear treatment or interfacial stabilizer between the two MAG emulsions $(\mathrm{p}>0.05)$, implying similar dye diffusion through the agar. 


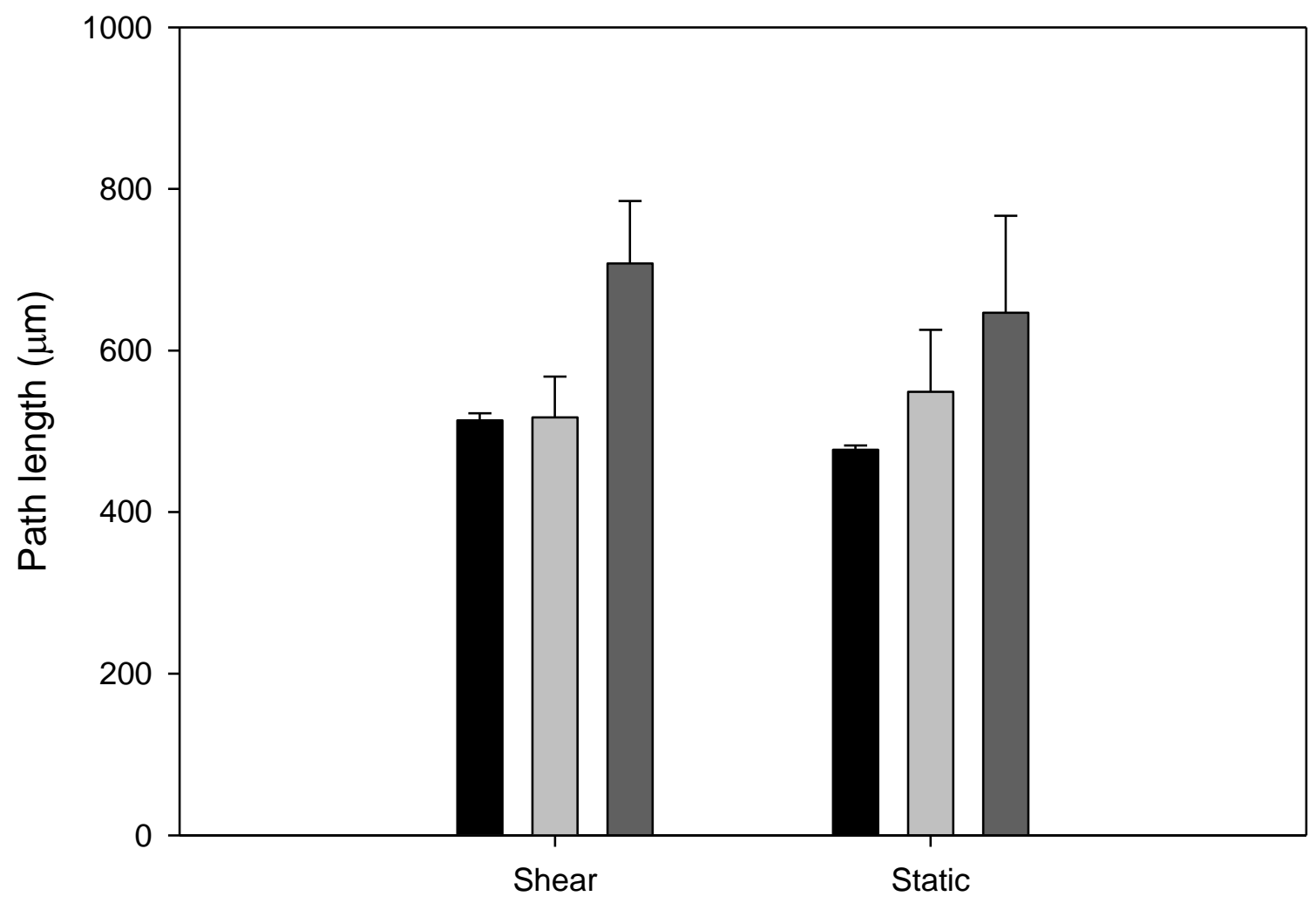

Figure 4.7: Rhodamine path length given in $\mu \mathrm{m}$ from the gel surface. Each emulsifier is identified: $\mathrm{GMO}=$ black; GMS = light grey; PGPR = dark grey.

The area close to the emulsion-agar interface $(\operatorname{Int}+50)$ was examined to determine change in local dye concentration. The PGPR-based emulsion exhibited a significantly higher dye concentration than the MAG-stabilized emulsions, and with higher values under shear than static conditions. There was no difference between the int+50 values of the GMO and GMS irrespective of shear conditions $(\mathrm{p}<0.05)$. Under shear and quiescent conditions, the GMO and GMS int+50 concentrations were $0.44-0.47 \mathrm{ppm}$. By contrast, the PGPR emulsions under shear released more Rhodamine $(\mathrm{p}<0.05)$ than during static exposure $(0.85 \pm 0.03$ and $0.67 \pm 0.07 \mathrm{ppm}$, respectively). Plots of the average intensity vs sample depth (i.e., y-axis position from the surface) (Appendix 
figure 4.1) were fitted to a Gaussian distribution, however model coefficients did not follow any discernable trend.

Emulsions were loaded with $20 \mathrm{~g}$ aqueous phase/100 g emulsion containing $0.2 \mathrm{mmol} / \mathrm{L}$ Rhodamine, or a 95 ppm aqueous phase concentration. Total Rhodamine B released was expressed as encapsulation efficiency (EE, equation 4.1):

$$
E E=\frac{\text { mass rhodamine in agar after exposure period }}{\text { mass rhodamine in applied emulsion }}
$$

After application to the agar surface and trimming, a total of $9.4 \mu \mathrm{m}$ of Rhodamine B was applied at the start of each treatment, presuming $100 \%$ initial encapsulation of the dye. Both GMO and GMS emulsions exhibited EE percentages of $\sim 15 \%$ during the exposure period, irrespective of shear treatment $(\mathrm{p}=0.997)$. PGPR, under shear $(35 \%)$ and static $(27 \%)$ conditions released significantly more dye than the MAG-based emulsions $(\mathrm{p}<0.001)$. The total dye released results followed a similar trend to the Int +50 values above. It is important to note that since the rheometer setup used a parallel plate to apply shear, there is an increasing shear gradient from the centre of the plate to the edge. At the centre of the gel, it is known that the shear rate is theoretically zero, however, the imaged region captures an area that, on average does not experience zero-shear.

Figure 4.8 shows the proposed mechanism leading to dye release. With the PGPR-stabilized emulsions, direct droplet contact with the agar surface and subsequent coalescence at the aqueous agar slab surface promoted dye release (Binks et al., 2010). Similar behaviour has been observed in double emulsions where, if more internal droplets are available close to the film separating the external outer phase, more of the encapsulated compound is released and at faster rates (Pays et al., 2002). Conversely, enhanced droplet stability with the MAG-stabilized emulsions hindered transport of the dispersed water phase into the agar gel. Shell porosity perhaps allowed for dye 
diffusion, if areas of the crystalline surface were either thin, absent or incomplete (Dan, 2016; Simovic and Prestidge, 2007). Minor defects in in otherwise solid interface would explain the nominal release of dye during static and shear exposure, and the similar EE, Int+50 and path length values for the GMS and GMO droplets during static and shear. The presence of the solid MAG shell surrounding the droplets not only contributed to an increase in measured SFC but also viscosity (Rafanan and Rousseau, 2019a), which may have further hindered dye diffusion. With the added stabilizing effect of aggregation in GMS and GMO-stabilized emulsions, the dye was more efficiently encapsulated under shear as more energy was required to break up droplet flocs to bring the droplets to the agar surface (Winuprasith et al., 2018). 

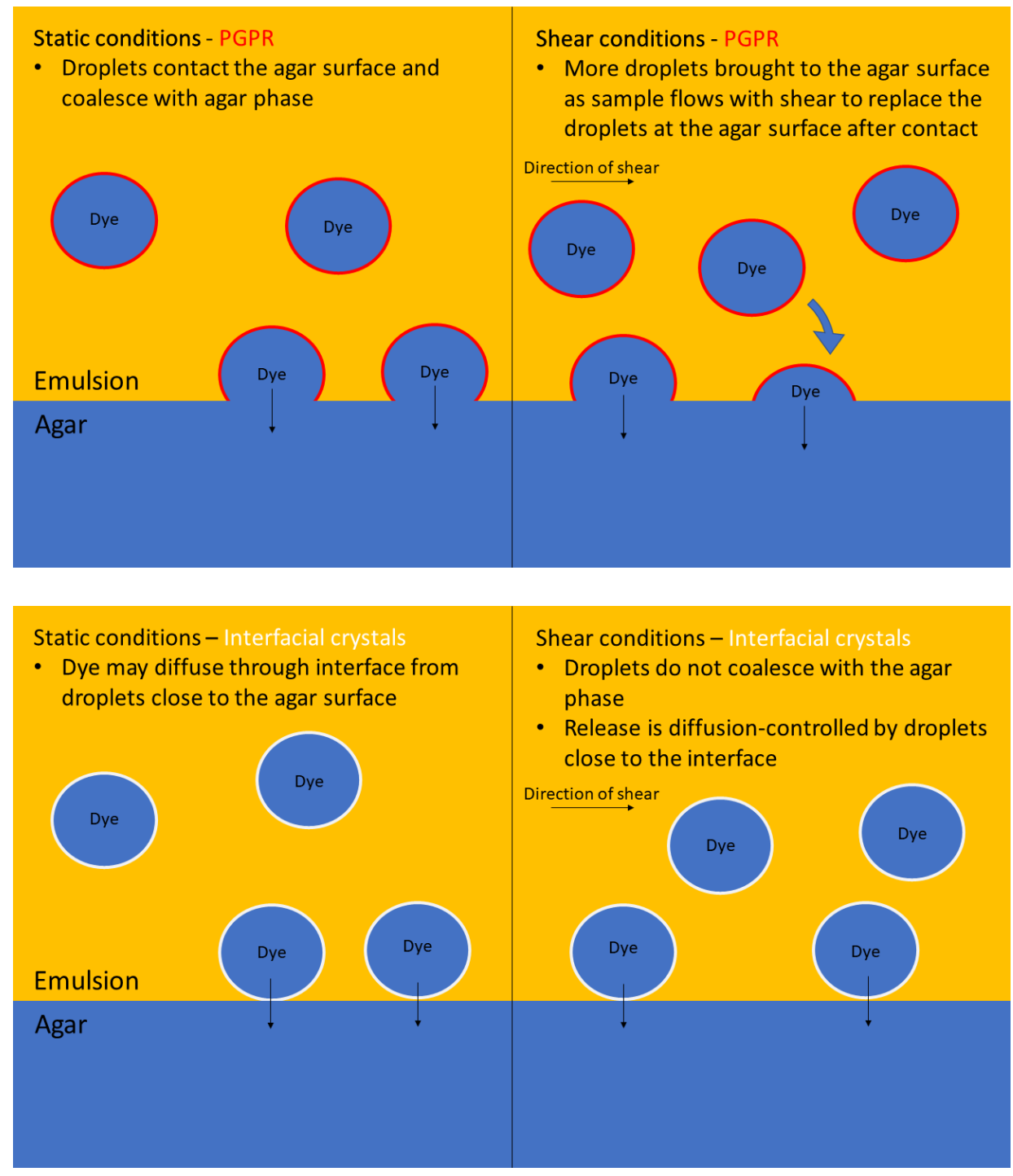

Figure 4.8 Release mechanism of dye from water droplets in a fat crystal network. The liquid state polymeric surfactant (PGPR) is shown in the top two images. Droplets stabilized with interfacial crystals (MAGs or HSO) are shown in the two bottom images.

Overall, dye within the PGPR emulsion droplets was more susceptible to release under shear because its lower viscosity allowed for more droplets to reach the agar-emulsion interface, where the droplets coalesced with the agar, releasing their cargo and likely redistributing the surfactant to the bulk oil phase. Dye encapsulated within MAG-stabilized emulsions droplets, however, was 
less prone to release given the solid nature of the interfacial film and increased viscosity, which limited droplets from directly contacting the agar-emulsion interface.

\subsection{Conclusion}

We describe a simple, time-effective method to determine the release of water-soluble compounds from emulsions under shear and static conditions. The present experimental results support the claim that emulsions stabilized with a solid crystalline shell are more effective vehicles for encapsulation since the adsorbed fat crystals provide an interface that is mechanically resistant to shear compared to emulsions stabilized with a liquid-state surfactant. Dye diffusion from the PGPR-stabilized emulsion was ultimately controlled by droplet interfacial structure, with shear enhancing this effect by increasing the number of droplets in the vicinity of the agar-emulsion boundary. For both MAG-stabilized emulsions (GMS and GMO), we speculate that impingement from growing crystals resulted in imperfections along the grain boundaries that serve as exit points for the dye towards the agar. This study experimentally supports the claim that shell-covered droplets provide superior encapsulation potential over liquid surfactant-stabilized emulsions under both shear and static conditions. Findings from this study can be applied to better target product development where the droplet interface can be designed to provide controlled encapsulation and release depending on the expected conditions of storage and use.

\section{General Conclusions and Further Research}

The first objective of this research was to determine whether structuring droplet interfaces (liquid surfactant interface or interfacial crystals) could exhibit different filler effects on the viscoelastic behaviour of fat crystal network-stabilized emulsions in the same manner as solid fillers can. It was shown in Chapter 2 that rheological behaviour in emulsions stabilized by a fat crystal network 
could be influenced primarily by the ability of the droplets to interact with surrounding fat crystal aggregates. A solid crystalline interface (MAG) promoted strong interactions between the droplets and their surroundings. These strong interactions resulted in an increase in $\mathrm{G}^{\prime}$ and hence could be considered an active filler. A critical aqueous phase volume fraction of $15 \mathrm{wt} \%$ or greater resulted in an increase in $\mathrm{G}^{\prime}$ and maintained predominantly elastic behaviour $(\tan \delta<1)$ at high frequency oscillations $(>1 \mathrm{~Hz})$ within the LVR. Below this threshold $(<15 \mathrm{wt} \%$ aqueous phase), no increase in elastic modulus was observed and the material became predominantly viscous with increasing frequency $(\tan \delta>1$ at frequencies above $1 \mathrm{~Hz}$ ). Conversely, droplets with a liquid interface (PGPR) behaved as inactive fillers as they had no effect on $\mathrm{G}^{\prime}$ and exhibited a $\tan \delta>1$ at higher frequencies irrespective of the water volume fraction. This was attributed to weak droplet-droplet interactions as well as those between droplets and neighboring crystal aggregates. The work here describes water droplets within a fat crystal network-stabilized emulsion as tunable fillers based on the strength and number of attractive interactions between the droplet interface and its surroundings.

Chapter 3 showed that droplet size distribution also affects the viscoelastic behaviour fat crystal network-stabilized emulsions, but this effect varied between liquid and solid interfaces. Strongly interacting filler droplets stabilized by MAGs at the interface promoted higher reinforcement and viscosity with smaller droplet sizes. The total number of attractive interactions between fillers and their surroundings increased with the increase in surface area, thus resulting in higher reinforcement and viscosity. Conversely, weakly interacting droplets (i.e., PGPR-stabilized droplets) exhibited higher reinforcement and viscosity with larger droplet size distributions, but to a lesser degree than the MAG-stabilized droplets. The larger, non-interacting droplets contributed to the overall dispersed matter within the oil phase, rather than interact strongly with the 
surrounding network, which impeded shear flow. Small, non-interacting droplets, however, moved freely within the interstices between fat crystal aggregates and did not contribute greatly to structural reinforcement or viscosity. These results demonstrated that while small droplet size distributions increased the possible number of interaction points, only strongly attractive interactions resulted in high degrees of reinforcement and viscosity increase.

The encapsulation behaviour of filler droplets in the presence of shear was examined in Chapter 4. Shear-mediated release of an encapsulated dye from PGPR-stabilized droplets increased with the application of shear. PGPR-stabilized droplets adjacent to the agar-emulsion interface coalesced with the agar, releasing the encapsulated dye. The lower apparent viscosity of the PGPR emulsion during shear may have also brought more dye-loaded droplets to the release mediumemulsion boundary. This may have facilitated diffusion of the dye into the release medium. However, shear did not affect the release of dye from MAG-stabilized droplets. The higher apparent viscosity of the MAG-stabilized emulsions caused by the strong interaction of droplets and their surroundings prevented droplet migration to the release medium. Solid interfaces appeared to be mechanically robust and remained intact with applied shear, keeping the dye within and exhibiting no change compared to dye release from MAG-stabilized droplet under static conditions. This demonstrated that interfacial crystal-stabilized droplets were not only stable under quiescent conditions, but also with shear.

While the results of these experiments have shown that droplet surfaces are functional components that can determine rheological behaviour and shear stability, further research can help clarify the nature of the attractive interactions resulting in reinforcement. High resolution TEM imaging of MAG-stabilized droplet interfaces may elucidate droplet surface roughness and may account for the difference in reinforcement seen between GMO and GMS at droplet sizes. Also, determination 
of the attractive force between droplets and crystal aggregates may provide valuable insight into how filler droplets interact with the surrounding network. This would require method development using a cantilevered capillary force apparatus to measure the attractive forces between two approaching particles, which in this case, would be a MAG-stabilized droplet and a single fat crystal aggregate. A method using a cantilevered capillary force apparatus is capable of determining the interaction strength between droplets. This method involves affixing a single droplet onto two capillaries; one "rigid" and one cantilevered. The cantilever is L-shaped and acts as a transducer to record the shifts in distance when brought close to the rigid cantilever. The movement of the cantilever is mediated by the attractive/repulsive forces between the droplets affixed to the ends of the cantilevers. This method closely resembles atomic force microscopy determination of attractive and repulsive forces between particles, but this technique is better suited for the larger sized particles, i.e.,, emulsion droplets (Frostad et al., 2014, 2013). This has been performed previously with droplets of various oils in water to measure the adhesion energy between two approaching droplets (Frostad et al., 2013). This, if applied to water droplets stabilized by different interfaces (solid and liquid), would provide valuable information regarding adhesion energy and thus, aggregation potential. The studies described in this body of work have successfully shown that there is a functional aspect to emulsion droplets that pertains to the structure at the interface. Further research into measuring the forces governing filler activity would elucidate this functional aspect of emulsion droplets within a network and relate the observed rheological changes to the forces acting between droplets and their surroundings. 


\section{Appendices}

\section{Appendix: Chapter 2}

Appendix table 2.1 - Droplet size distributions for all fat crystal-stabilized emulsions as determined by bright field microscopy image analysis. Average droplet diameter was determined by using the estimated average value of the lognormal frequency distribution.

\begin{tabular}{|c|c|c|c|c|c|c|}
\hline \multirow{3}{*}{$\frac{\text { Water }(w t \%)}{5}$} & \multicolumn{6}{|c|}{ GMO } \\
\hline & \multicolumn{3}{|c|}{$\begin{array}{c}\text { Day 0 } \\
\text { Droplet diameter } \\
(\mu \mathrm{m})\end{array}$} & \multicolumn{3}{|c|}{$\begin{array}{c}\text { Day } 7 \\
\text { Droplet diameter } \\
(\mu \mathrm{m})\end{array}$} \\
\hline & 1.2 & \pm & 0.5 & 2.2 & \pm & 0.2 \\
\hline 10 & 0.7 & \pm & 0.1 & 0.8 & \pm & 0.0 \\
\hline 15 & 0.9 & \pm & 0.1 & 1.0 & \pm & 0.3 \\
\hline 20 & 0.7 & \pm & 0.0 & 0.9 & \pm & 0.0 \\
\hline
\end{tabular}

\begin{tabular}{ccccccc}
\hline & PGPR & \multicolumn{3}{c}{\begin{tabular}{c} 
Day 7 \\
\cline { 2 - 7 } Water (wt \%)
\end{tabular}} & $\begin{array}{c}\text { Day 0 } \\
\text { Droplet diameter } \\
(\mu \mathrm{m})\end{array}$ & \multicolumn{3}{c}{$\begin{array}{c}\text { Droplet diameter } \\
(\mu \mathrm{m})\end{array}$} \\
\hline $\mathbf{5}$ & 1.7 & \pm & 0.1 & 0.8 & \pm & 0.1 \\
$\mathbf{1 0}$ & 2.9 & \pm & 0.3 & 0.8 & \pm & 0.0 \\
$\mathbf{1 5}$ & 0.9 & \pm & 0.0 & 0.8 & \pm & 0.1 \\
$\mathbf{2 0}$ & 1.4 & \pm & 1.2 & 0.9 & \pm & 0.0 \\
\hline
\end{tabular}


Appendix table 2.2 - Peak melting temperatures $\left({ }^{\circ} \mathrm{C}\right)$ of $\mathrm{HSO}$ in the $\mathrm{W} / \mathrm{O}$ emulsions as a function of time, water content and emulsifier type. Different lower-case superscript letters denote withincolumn statistical differences in SFC as a function of water content. Different upper-case superscript letters denote between-column statistical differences in SFC as a function of time for each emulsifier $(n=3)$.

\begin{tabular}{ccccc}
\hline & \multicolumn{2}{c}{ GMO } & \multicolumn{2}{c}{ PGPR } \\
Water (wt \%) & Day 0 & Day 7 & Day 0 & Day 7 \\
\hline $\mathbf{0}$ & $55.4 \pm 0.1^{\mathrm{aA}}$ & $55.8 \pm 0.0^{\mathrm{aB}}$ & $55.5 \pm 0.0^{\mathrm{aA}}$ & $55.6 \pm 0.3^{\mathrm{aA}}$ \\
$\mathbf{5}$ & $54.5 \pm 0.1^{\mathrm{bA}}$ & $54.9 \pm 0.2^{\mathrm{bB}}$ & $55.3 \pm 0.0^{\mathrm{aA}}$ & $55.2 \pm 0.2^{\mathrm{aA}}$ \\
$\mathbf{1 0}$ & $54.7 \pm 0.0^{\mathrm{bA}}$ & $54.6 \pm 0.1^{\mathrm{bA}}$ & $54.7 \pm 0.0^{\mathrm{bA}}$ & $55.4 \pm 0.0^{\mathrm{aB}}$ \\
$\mathbf{1 5}$ & $54.5 \pm 0.2^{\mathrm{bA}}$ & $54.4 \pm 0.4^{\mathrm{bA}}$ & $55.6 \pm 0.1^{\mathrm{aA}}$ & $55.2 \pm 0.1^{\mathrm{aB}}$ \\
$\mathbf{2 0}$ & $54.7 \pm 0.5^{\mathrm{bA}}$ & $54.4 \pm 0.6^{\mathrm{bA}}$ & $55.8 \pm 0.2^{\mathrm{cA}}$ & $55.4 \pm 0.2^{\mathrm{aA}}$ \\
\hline
\end{tabular}


Appendix figure 2.1 - Frequency sweep data from $0.01-10 \mathrm{~Hz}$ at $0.02 \%$ strain for fat crystal network stabilized emulsions. Water concentration: A - Blank, B - $5 \mathrm{wt} \%, \mathrm{C}-10 \mathrm{wt} \%, \mathrm{D}-15$ $\mathrm{wt} \%, \mathrm{E}-20 \mathrm{wt} \%$. Viscoelastic parameters: Closed symbols - G', open symbols - G". Symbols as follows: •-GMO G' Day 0, o- GMO G" Day 0, $\boldsymbol{\nabla}$ - GMO G' Day 7, $\Delta-$ GMO G" Day 7, PGPR G' Day 0, $\square-$ PGPR G" Day 0, - PGPR G' Day 7, $\diamond-$ PGPR G" Day 7. Error bars removed for clarity $(\mathrm{n}=3)$.
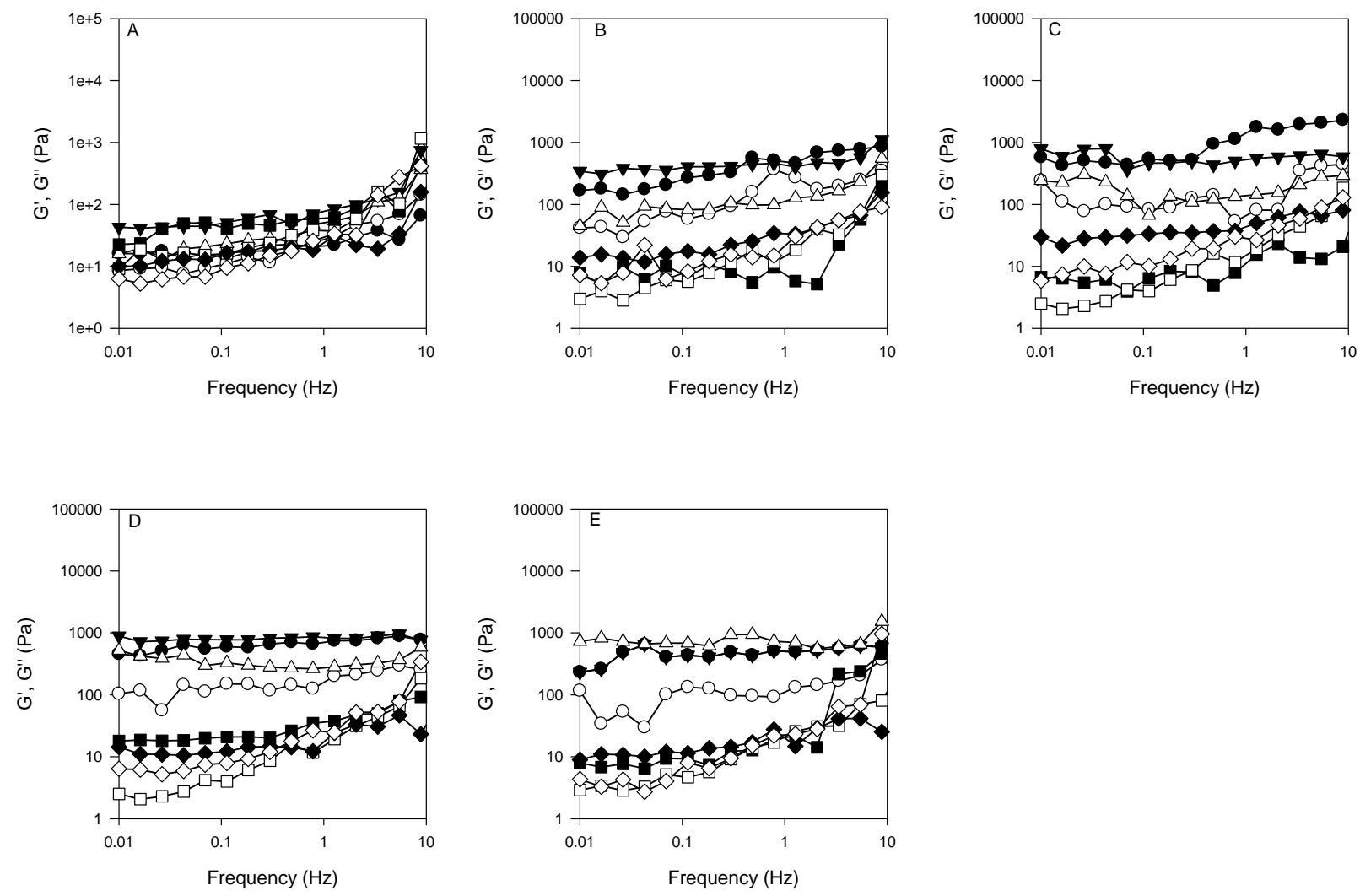


\section{Appendix: Chapter 3}

Appendix figure 3.1 - XRD scattering of different HSO polymorphs. Major peaks for each polymorph are labeled.

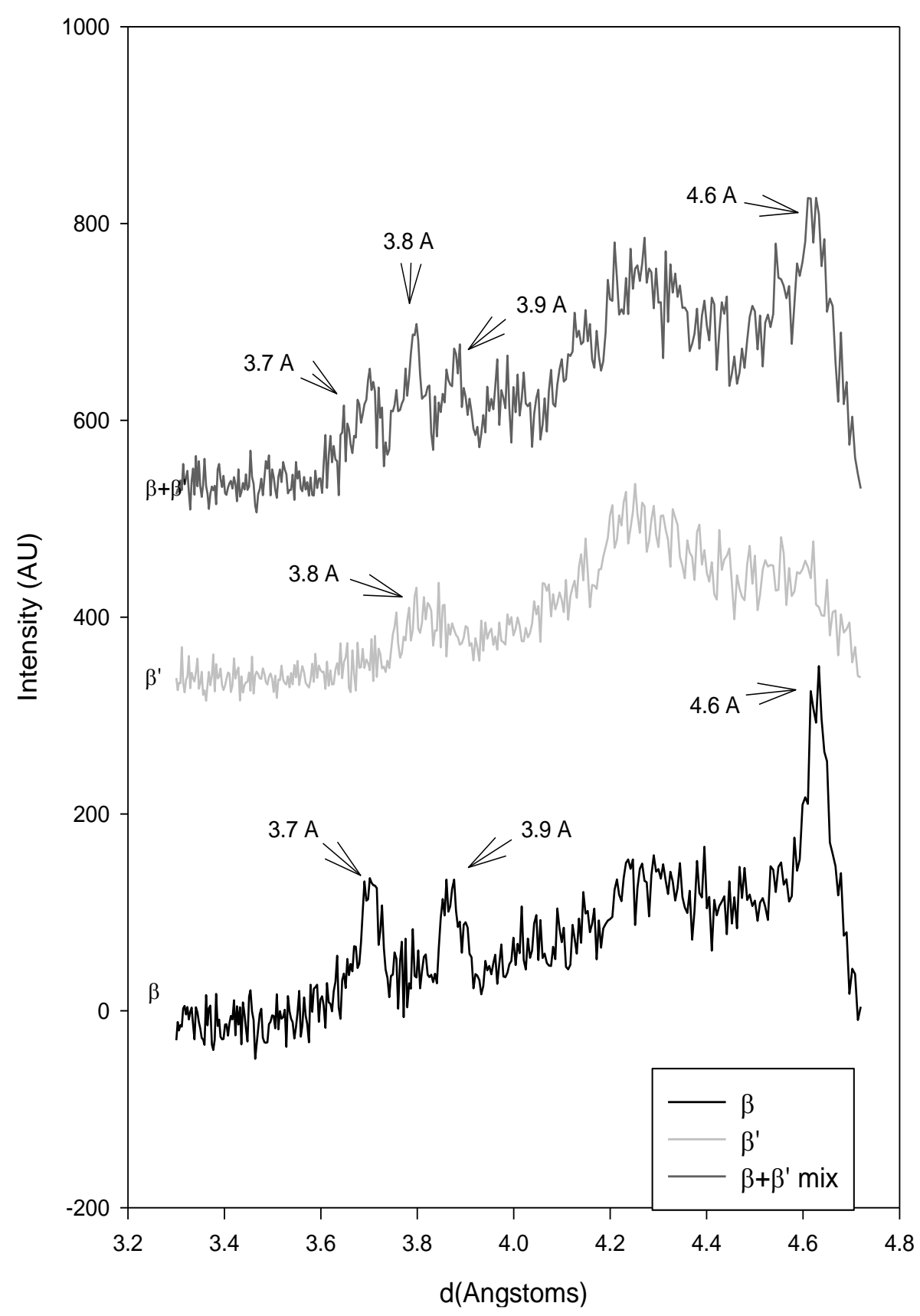


Appendix table 3.1 - Power law fitting of emulsion viscosity - $a$ coefficient

\begin{tabular}{|c|c|c|c|c|c|c|c|c|}
\hline & \multirow{2}{*}{$\begin{array}{l}\text { Day } \\
0\end{array}$} & \multicolumn{2}{|c|}{ PGPR } & \multicolumn{2}{|c|}{ GMS } & \multicolumn{3}{|c|}{ GMO } \\
\hline \multirow{5}{*}{ Blank } & & 4 & \pm 0 & 47 & \pm 54 & 20 & \pm & 8 \\
\hline & 7 & 7 & \pm 2 & 52 & \pm 69 & 346 & \pm & 44 \\
\hline & 14 & 6 & \pm 5 & 60 & \pm 73 & 355 & \pm & 27 \\
\hline & 21 & 4 & \pm 0 & 96 & \pm 91 & 310 & \pm & 40 \\
\hline & 28 & 3 & \pm 1 & 133 & \pm 77 & 269 & \pm & 42 \\
\hline \multirow{5}{*}{ Small DSD } & 0 & 19 & \pm 8 & 461 & \pm 216 & 141 & \pm & 77 \\
\hline & 7 & & \pm 2 & 519 & \pm 122 & 191 & \pm & 100 \\
\hline & 14 & 17 & \pm 5 & 670 & \pm 226 & 210 & \pm & 118 \\
\hline & 21 & 16 & \pm 4 & 548 & \pm 147 & 209 & \pm & 142 \\
\hline & 28 & 15 & \pm 3 & 562 & \pm 54 & 248 & \pm & 152 \\
\hline \multirow{5}{*}{ Large DSD } & 0 & 55 & \pm 48 & 422 & \pm 103 & 96 & \pm & 30 \\
\hline & 7 & 22 & \pm 12 & 271 & \pm 86 & 337 & \pm & 337 \\
\hline & 14 & 39 & \pm 37 & 359 & \pm 144 & 168 & \pm & 44 \\
\hline & 21 & & - & 410 & \pm 94 & 175 & \pm & 27 \\
\hline & 28 & & - & 392 & \pm 52 & 254 & \pm & 153 \\
\hline
\end{tabular}


Appendix table 3.2 - Power law fitting of emulsion viscosity $-b$ coefficient

\begin{tabular}{|c|c|c|c|c|c|c|c|c|c|}
\hline & Day & PGPR & & & GMS & & GMO & & \\
\hline \multirow{5}{*}{ Blank } & 0 & 0.7 & \pm & $0.02^{\mathrm{D}}$ & 0.2 & \pm 0.28 & 0.5 & \pm & $0.08^{\mathrm{A}}$ \\
\hline & 7 & 0.6 & \pm & $0.20^{\mathrm{k}}$ & 0.3 & $\pm 0.43^{\mathrm{B}, \mathrm{C}}$ & -0.4 & \pm & 0.09 \\
\hline & 14 & 0.6 & \pm & $0.32^{1}$ & 0.1 & \pm 0.40 & -0.0 & \pm & 0.78 \\
\hline & 21 & 0.7 & \pm & $0.06^{\mathrm{G}}$ & -0.1 & \pm 0.07 & -0.5 & \pm & 0.25 \\
\hline & 28 & 0.8 & \pm & $0.05^{\mathrm{H}}$ & -0.5 & $\pm 0.58^{\mathrm{B}}$ & -0.4 & \pm & $0.04^{\mathrm{A}}$ \\
\hline \multirow{5}{*}{ Small DSD } & 0 & 0.4 & \pm & $0.18^{\mathrm{D}}$ & -0.3 & $\pm 0.11^{\mathrm{f}}$ & 0.3 & \pm & $0.13^{\mathrm{a}}$ \\
\hline & 7 & 0.5 & \pm & $0.04^{\mathrm{k}}$ & -0.3 & $\pm 0.13^{\mathrm{C}, \mathrm{g}}$ & 0.2 & \pm & $0.12^{b}$ \\
\hline & 14 & 0.5 & \pm & $0.12^{1}$ & -0.3 & $\pm 0.07^{\mathrm{h}}$ & 0.2 & \pm & $0.16^{\mathrm{c}}$ \\
\hline & 21 & 0.8 & \pm & $0.10^{\mathrm{G}}$ & -0.2 & $\pm 0.07^{\mathrm{i}}$ & 0.2 & \pm & $0.13^{\mathrm{d}}$ \\
\hline & 28 & 0.5 & \pm & $0.04^{\mathrm{H}}$ & -0.4 & $\pm 0.25^{\mathrm{j}}$ & 0.1 & \pm & $0.15^{\mathrm{e}}$ \\
\hline \multirow{5}{*}{ Large DSD } & 0 & -0.3 & \pm & $0.81^{\mathrm{D}}$ & -0.3 & $\pm 0.19^{f}$ & 0.3 & \pm & $0.06^{\mathrm{a}}$ \\
\hline & 7 & 0.6 & \pm & $1.20^{\mathrm{E}}$ & -0.1 & $\pm 0.08^{\mathrm{g}}$ & 0.1 & \pm & $0.26^{b}$ \\
\hline & 14 & -1 & \pm & $2.35^{\mathrm{F}}$ & -0.2 & $\pm 0.15^{\mathrm{h}}$ & 0.2 & \pm & $0.02^{\mathrm{c}}$ \\
\hline & 21 & & - & & -0.3 & $\pm 0.06^{\mathrm{i}}$ & 0.2 & \pm & $0.05^{\mathrm{d}}$ \\
\hline & 28 & & - & & -0.3 & $\pm 0.16^{\mathrm{j}}$ & 0.1 & \pm & $0.16^{\mathrm{e}}$ \\
\hline
\end{tabular}




\section{Appendix: Chapter 4}

Appendix figure 4.1 - Average dye intensity within the gel as a function of distance from the gel surface which begins at $x=0$. The dashed vertical line near the $y$-axis denotes the start of the int +50 location. The dotted horizontal line corresponds to the signal threshold $(y=20)$ below which there is no detection of dye. PGPR = dark grey, GMO = black and GMS = light grey. Sheartreated samples are solid lines while the long-dashed curves are static exposure conditions.

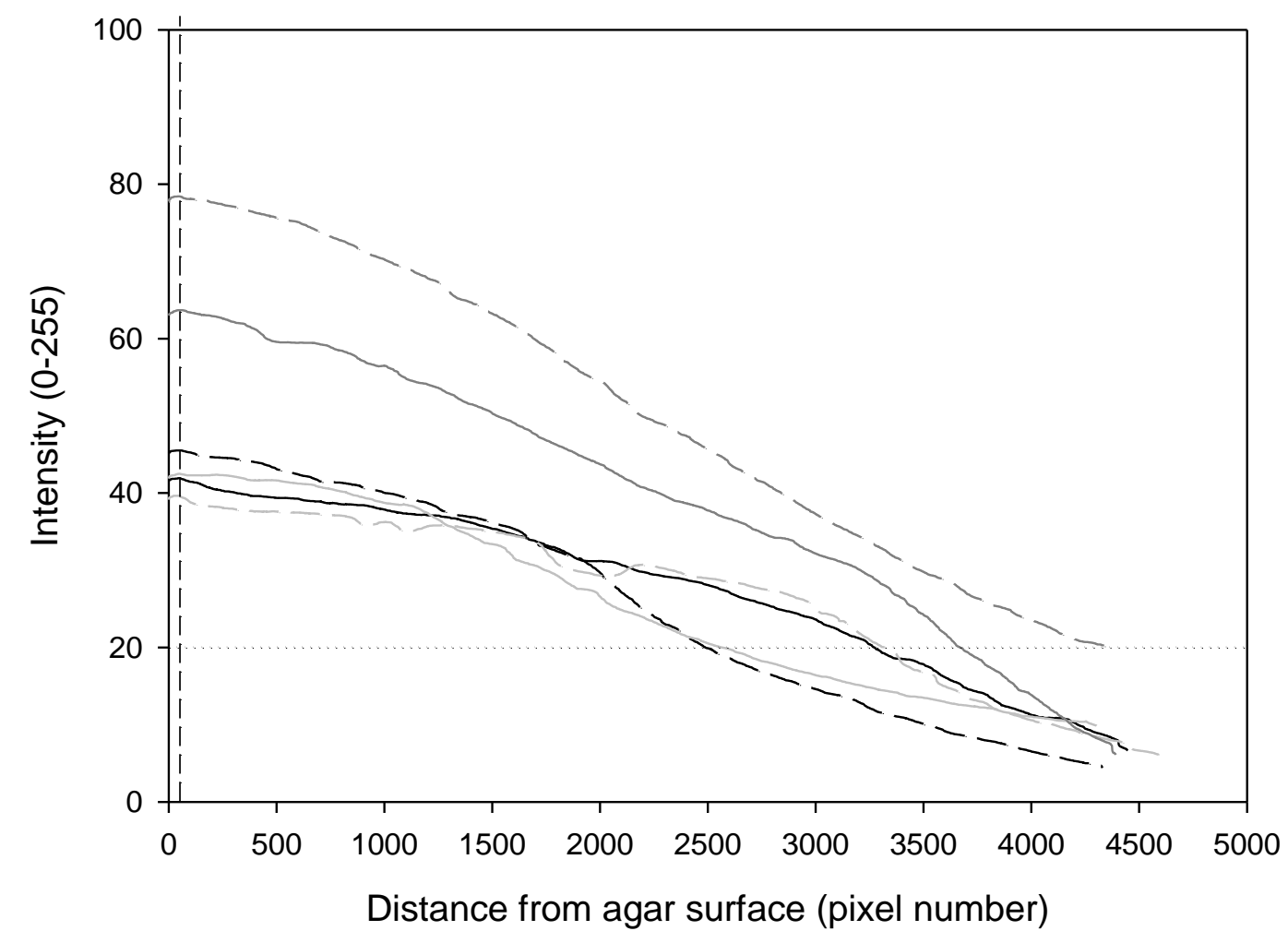


Appendix figure 4.2 - Melting curves of the PGPR, GMS and GMO stabilized emulsions determined by DSC at a heating rate of $5^{\circ} \mathrm{C} / \mathrm{min}$.

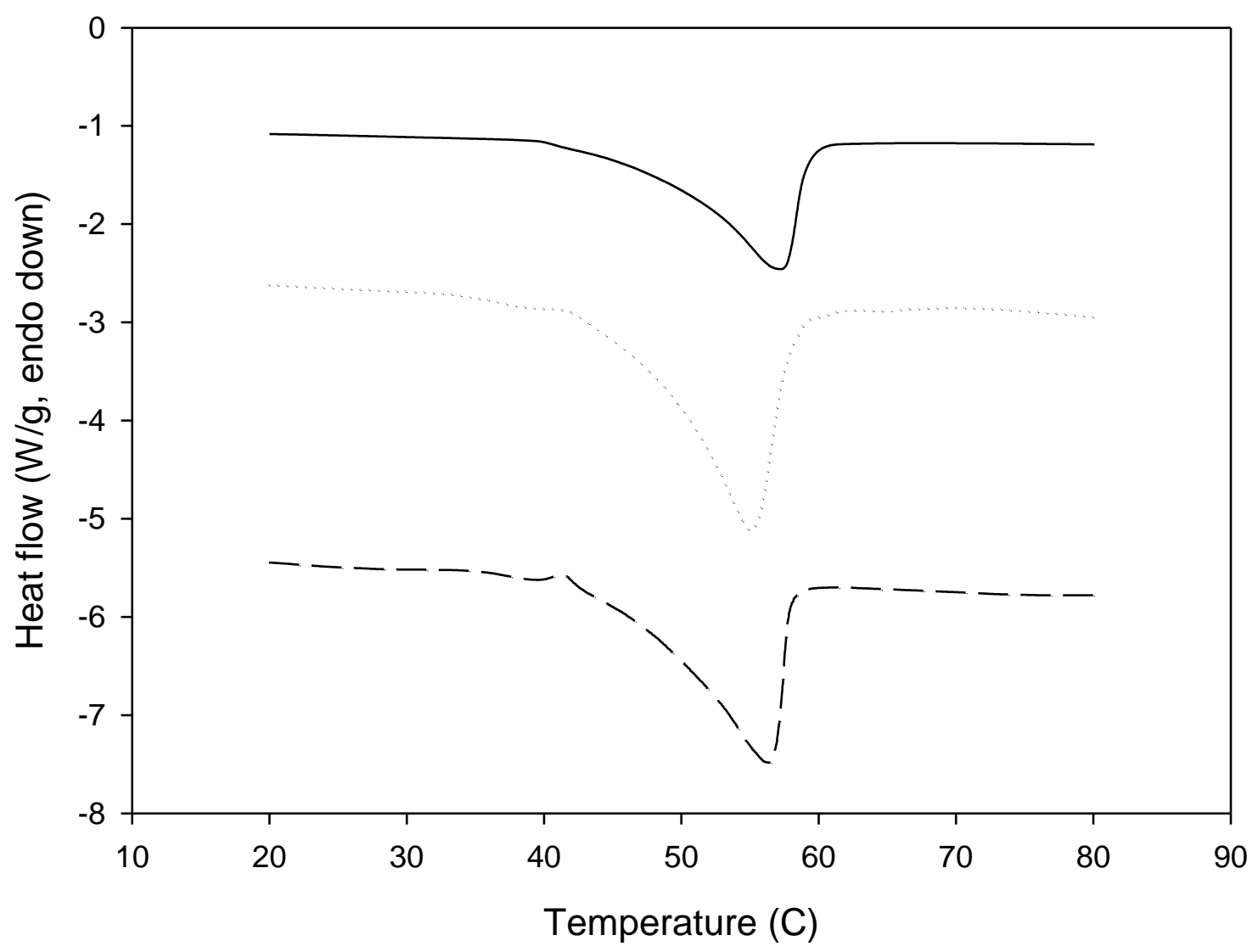




\section{References}

Afoakwa, E.O., Paterson, A., Fowler, M., 2008. Effects of particle size distribution and composition on rheological properties of dark chocolate. Eur. Food Res. Technol. 226, 1259-1268. https://doi.org/10.1007/s00217-007-0652-6

Alvarez-Román, R., Naik, A., Kalia, Y.N., Fessi, H., Guy, R.H., 2004. Visualization of skin penetration using confocal laser scanning microscopy. Eur. J. Pharm. Biopharm. 58, 301316. https://doi.org/10.1016/j.ejpb.2004.03.027

Anton, M., Le Denmat, M., Beaumal, V., Pilet, P., 2001. Filler effects of oil droplets on the rheology of heat-set emulsion gels prepared with egg yolk and egg yolk fractions. Colloids Surf. B. Biointerfaces 21, 137-147.

Aronhime, J.S., Sarig, S., Garti, N., 1988. Dynamic control of polymorphic transformation in triglycerides by surfactants: The button syndrome. J. Am. Oil Chem. Soc. 65, 1144-1150. https://doi.org/10.1007/BF02660571

Aveyard, R., Binks, B.P., Clint, J.H., 2003. Emulsions stabilised solely by colloidal particles. Adv. Colloid Interface Sci. 100-102, 503-546. https://doi.org/10.1016/S00018686(02)00069-6

Barnes, H.A., 2003. A Review of the Rheology of Filled Viscoelastic Systems. Rheol. Rev. 2003, 1-36. https://doi.org/10.1081/ASR-120017479

Barnes, H.A., 1994. Rheology of emulsions - A review. Colloids Surfaces A Physicochem. Eng. Asp. 91, 89-95. https://doi.org/10.1016/0927-7757(93)02719-U

Bell, A., Gordon, M.H., Jirasubkunakorn, W., Smith, K.W., 2007. Effects of composition on fat rheology and crystallisation. Food Chem. 101, 799-805. https://doi.org/10.1016/j.foodchem.2006.02.033

Beri, A., Norton, J.E., Norton, I.T., 2013. Effect of emulsifier type and concentration, aqueous phase volume and wax ratio on physical, material and mechanical properties of water in oil lipsticks. Int. J. Cosmet. Sci. 35, 613-621. https://doi.org/10.1111/ics.12085

Binks, B.P., 2002. Particles as surfactants - Similarities and differences. Curr. Opin. Colloid Interface Sci. 7, 21-41. https://doi.org/10.1016/S1359-0294(02)00008-0

Binks, B.P., Fletcher, P.D.I., Holt, B.L., Beaussoubre, P., Wong, K., 2010. Selective retardation of perfume oil evaporation from oil-in-water emulsions stabilized by either surfactant or nanoparticles. Langmuir 26, 18024-18030. https://doi.org/10.1021/la103700g

Brummer, R., Godersky, S., 1999. Rheological studies to objectify sensations occurring when cosmetic emulsions are applied to the skin. Colloids Surfaces A Physicochem. Eng. Asp. 152, 89-94. https://doi.org/10.1016/S0927-7757(98)00626-8

Campos, R., Narine, S.S., Marangoni, A.G., 2002. Effect of cooling rate on the structure and mechanical properties of milk fat and lard. Food Res. Int. 35, 971-981. https://doi.org/10.1016/S0963-9969(02)00159-X 
Chen, D.T.N., Wen, Q., Janmey, P.A., Crocker, J.C., Yodh, A.G., 2010. Rheology of Soft Materials. Annu. Rev. Condens. Matter Phys. 1, 301-322. https://doi.org/10.1146/annurevconmatphys-070909-104120

Chen, J., Dickinson, E., 1999. Effect of surface character of filler particles on rheology of heatset whey protein emulsion gels. Colloids Surfaces B Biointerfaces 12, 373-381. https://doi.org/10.1016/S0927-7765(98)00091-5

Chen, J., Dickinson, E., 1995a. Protein surfactant interfacial interactions - Part 2: Electrophoretic mobility of mixed protein and surfactant systems. Colloids Surfaces A Physicochem. Eng. Asp. 100, 267-277.

Chen, J., Dickinson, E., 1995b. Protein surfactant interfacial interactions - Part I: Flocculation of emulsions containing mixed protein and surfactant. Colloids Surfaces A Physicochem. Eng. Asp. 100, 155-265.

Chevalier, Y., Bolzinger, M.A., 2013. Emulsions stabilized with solid nanoparticles: Pickering emulsions. Colloids Surfaces A Physicochem. Eng. Asp. 439, 23-34. https://doi.org/10.1016/j.colsurfa.2013.02.054

Clark, D.B., Lenain, L., Feddersen, F., Boss, E., Guza, R.T., 2014. Aerial imaging of fluorescent dye in the near shore. J. Atmos. Ocean. Technol. 31, 1410-1421. https://doi.org/10.1175/JTECH-D-13-00230.1

Da Silva, E., Bresson, S., Rousseau, D., 2009. Characterization of the three major polymorphic forms and liquid state of tristearin by Raman spectroscopy. Chem. Phys. Lipids 157, 113119. https://doi.org/10.1016/j.chemphyslip.2008.11.002

Dan, N., 2016. Transport and release in nano-carriers for food applications. J. Food Eng. 175, 136-144. https://doi.org/10.1016/j.jfoodeng.2015.12.017

de Man, J.M., Beers, A.M., 1988. Fat Crystal Networks: Structure and Rheological Properties. J. Texture Stud. 18, 303-318.

Dedinaite, A., Campbell, B., 2000. Interactions between mica surfaces across triglyceride solution containing phospholipid and polyglycerol polyricinoleate. Langmuir 16, 22482253. https://doi.org/10.1021/la991018u

Di Bari, V., Macnaughtan, W., Norton, J., Sullo, A., Norton, I., 2017. Crystallisation in water-incocoa butter emulsions: Role of the dispersed phase on fat crystallisation and polymorphic transition. Food Struct. 12, 82-93. https://doi.org/10.1016/j.foostr.2016.10.001

Dias, J., Alvarenga, N., Sousa, I., 2015. Effect of hydrocolloids on low-fat chocolate fillings. J. Food Sci. Technol. 52, 7209-7217. https://doi.org/10.1007/s13197-015-1841-0

Dickinson, E., 2013. Stabilising emulsion-based colloidal structures with mixed food ingredients. J. Sci. Food Agric. 93, 710-721. https://doi.org/10.1002/jsfa.6013

Dickinson, E., Chen, J., 1999. Heat set whey protien emulsion gels: Role of Active and Inactive Filler Particles. J. Dispers. Sci. Technol. 20, 197-213.

Dickinson, E., Hong, S.-T., 1995. Influence of Water-Soluble Nonionic Emulsifier on the 
Rheology of Heat-Set Protein-Stabilized Emulsion Gels. J. Agric. Food Chem. 43, 2560 2566. https://doi.org/10.1021/jf00058a002

Do, T.A.L., Hargreaves, J.M., Wolf, B., Hort, J., Mitchell, J.R., 2007. Impact of particle size distribution on rheological and textural properties of chocolate models with reduced fat content. J. Food Sci. 72, 541-552. https://doi.org/10.1111/j.1750-3841.2007.00572.x

Douaire, M., Di Bari, V., Norton, J.E., Sullo, a., Lillford, P., Norton, I.T., 2014. Fat crystallisation at oil-water interfaces. Adv. Colloid Interface Sci. 203, 1-10. https://doi.org/10.1016/j.cis.2013.10.022

Duffus, L.J., Norton, J.E., Smith, P., Norton, I.T., Spyropoulos, F., 2016. A comparative study on the capacity of a range of food-grade particles to form stable $\mathrm{O} / \mathrm{W}$ and W/O Pickering emulsions. J. Colloid Interface Sci. 473, 9-21. https://doi.org/10.1016/j.jcis.2016.03.060

Farris, R.J., 1968. Prediction of the Viscosity of Multimodal Suspensions from Unimodal Viscosity Data. Trans. Soc. Rheol. 12, 281-301. https://doi.org/10.1122/1.549109

Foubert, I., Vanhoutte, B., Dewettinck, K., 2004. Temperature and concentration dependent effect of partial glycerides on milk fat crystallization. Eur. J. Lipid Sci. Technol. 106, 531539. https://doi.org/10.1002/ejlt.200400979

Frasch-Melnik, S., Norton, I.T., Spyropoulos, F., 2010. Fat-crystal stabilised w/o emulsions for controlled salt release. J. Food Eng. 98, 437-442. https://doi.org/10.1016/j.jfoodeng.2010.01.025

Fredrick, E., Foubert, I., Van Sype, J. De, Dewettinck, K., 2008. Influence of monoglycerides on the crystallization behavior of palm oil. Cryst. Growth Des. 8, 1833-1839. https://doi.org/10.1021/cg070025a

Frelichowska, J., Bolzinger, M.A., Pelletier, J., Valour, J.P., Chevalier, Y., 2009. Topical delivery of lipophilic drugs from o/w Pickering emulsions. Int. J. Pharm. 371, 56-63. https://doi.org/10.1016/j.ijpharm.2008.12.017

Fröhlich, J., Niedermeier, W., Luginsland, H.-D., 2005a. The effect of filler-filler and fillerelastomer interaction on rubber reinforcement. Compos. Part A Appl. Sci. Manuf. 36, 449460. https://doi.org/10.1016/j.compositesa.2004.10.004

Fröhlich, J., Niedermeier, W., Luginsland, H.-D.D., 2005b. The effect of filler-filler and fillerelastomer interaction on rubber reinforcement. Compos. Part A Appl. Sci. Manuf. 36, 449460. https://doi.org/10.1016/j.compositesa.2004.10.004

Frostad, J.M., Collins, M.C., Leal, L.G., 2013. Cantilevered-capillary force apparatus for measuring multiphase fluid interactions. Langmuir 29, 4715-4725. https://doi.org/10.1021/la304115k

Frostad, J.M., Seth, M., Bernasek, S.M., Leal, L.G., 2014. Direct measurement of interaction forces between charged multilamellar vesicles $\dagger$. Soft Matter 10, 7769-7780. https://doi.org/10.1039/c3sm52785a

Garbolino, C., Bartoccini, M., 2005. The influence of emulsifiers on the crystallisation behaviour of a palm oil-based blend 616-626. https://doi.org/10.1002/ejlt.200501186 
Garti, N., Binyamin, H., Aserin, a., 1998. Stabilization of water-in-oil emulsions by submicrocrystalline $\alpha$-form fat particles. J. Am. Oil Chem. Soc. 75, 1825-1831. https://doi.org/10.1007/s11746-998-0338-7

Genovese, D.B., 2012. Shear rheology of hard-sphere, dispersed, and aggregated suspensions, and filler-matrix composites. Adv. Colloid Interface Sci. 171-172, 1-16. https://doi.org/10.1016/j.cis.2011.12.005

Genovese, D.B., Ye, A., Singh, H., 2010. High methoxyl pectin/apple particles composite gels: Effect of particle size and particle concentration on mechanical properties and gel structure. J. Texture Stud. 41, 171-189. https://doi.org/10.1111/j.1745-4603.2010.00220.x

Ghosh, S., Rousseau, D., 2012. Triacylglycerol interfacial crystallization and shear structuring in water-in-oil emulsions. Cryst. Growth Des. 12, 4944-4954. https://doi.org/10.1021/cg300872m

Ghosh, S., Rousseau, D., 2011. Fat crystals and water-in-oil emulsion stability. Curr. Opin. Colloid Interface Sci. 16, 421-431. https://doi.org/10.1016/j.cocis.2011.06.006

Ghosh, S., Rousseau, D., 2009. Freeze-thaw stability of water-in-oil emulsions. J. Colloid Interface Sci. 339, 91-102. https://doi.org/10.1016/j.jcis.2009.07.047

Ghosh, S., Tran, T., Rousseau, D., 2011. Comparison of Pickering and network stabilization in water-in-oil emulsions. Langmuir 27, 6589-97. https://doi.org/10.1021/la200065y

Ghouchi Eskandar, N., Simovic, S., Prestidge, C.A., 2009. Nanoparticle coated submicron emulsions: Sustained in-vitro release and improved dermal delivery of all-trans-retinol. Pharm. Res. 26, 1764-1775. https://doi.org/10.1007/s11095-009-9888-0

Gregersen, S.B., Andersen, M.D., Hammershøj, M., Wiking, L., 2017. Impact of triacylglycerol composition on shear-induced textural changes in highly saturated fats. Food Chem. 215, 438-446. https://doi.org/10.1016/j.foodchem.2016.08.008

Haj-shafiei, S., Ghosh, S., Rousseau, D., 2013. Kinetic stability and rheology of wax-stabilized water-in-oil emulsions at different water cuts. J. Colloid Interface Sci. 410, 11-20. https://doi.org/10.1016/j.jcis.2013.06.047

Herrera, M.L., Hartel, R.W., 2000. Effect of processing conditions on crystallization kinetics of a milk fat model system. J. Am. Oil Chem. Soc. 77, 1177-1188. https://doi.org/10.1007/s11746-000-0184-4

Himawan, C., MacNaughtan, W., Farhat, I. a., Stapley, A.G.F., 2007. Polymorphic occurrence and crystallization rates of tristearin/tripalmitin mixtures under non-isothermal conditions. Eur. J. Lipid Sci. Technol. 109, 49-60. https://doi.org/10.1002/ejlt.200600179

Himawan, C., Starov, V.M., Stapley, A.G.F., 2006. Thermodynamic and kinetic aspects of fat crystallization. Adv. Colloid Interface Sci. 122, 3-33. https://doi.org/10.1016/j.cis.2006.06.016

Hodge, S.M., Rousseau, D., 2005. Continuous-phase fat crystals strongly influence water-in-oil emulsion stability. J. Am. Oil Chem. Soc. 82, 159-164. https://doi.org/10.1007/s11746-0055166-4 
Johansson, D., Bergenståhl, B., 1995. Sintering of fat crystal networks in oil during postcrystallization processes. J. Am. Oil Chem. Soc. 72, 911-920.

https://doi.org/10.1007/BF02542069

Johansson, D., Bergenståhl, B., 1992a. The influence of food emulsifers on fat and sugar dispersions in oils. I. Adsorption, Sedimentation. J. Am. Oil Chem. Soc. 69, 705-717. https://doi.org/10.1007/BF02635906

Johansson, D., Bergenståhl, B., 1992b. The influence of food emulsifers on fat and sugar dispersions in oils. II. Rheology, Colloidal Forces. J. Am. Oil Chem. Soc. 69, 718-727. https://doi.org/10.1007/BF02635906

Koenig, A., Hébraud, P., Perrin, P., 2002. Preparation and rheological properties of emulsion gels. Langmuir 18, 6458-6461. https://doi.org/10.1021/la0200663

Krieger, I.M., 1972. Rheology of monodisperse latices. Adv. Colloid Interface Sci. 3, 111-136.

Krog, N., Larsson, K., 1992. Crystallization a Interfaces in Food Emulsions - A General Phenomenon. Fat Sci. Technol. 94, 55-57.

Kumar, K., Nikolov, A.D., Wasan, D.T., 2001. Mechanisms of stabilization of water-in-crude oil emulsions. Ind. Eng. Chem. Res. 40, 3009-3014. https://doi.org/10.1021/ie000663p

Lawler, P.J., Dimick, P.S., 2002. Crystallization and Polymorphism of Fats, in: Akoh, C.C., Min, D.B. (Eds.), Food Lipids: Chemistry, Nutrition, and Biotechnology. CRC Press, Baton Rouge, LA, pp. 292-315.

Le Révérend, B.J.D., Taylor, M.S., Norton, I.T., 2011. Design and application of water-in-oil emulsions for use in lipstick formulations. Int. J. Cosmet. Sci. 33, 263-268. https://doi.org/10.1111/j.1468-2494.2010.00624.x

Lee, H.S., Singh, P., Thomason, W.H., Fogler, H.S., 2008. Waxy oil gel breaking mechanisms: Adhesive versus cohesive failure. Energy and Fuels 22, 480-487. https://doi.org/10.1021/ef700212v

Libster, D., Aserin, A., Yariv, D., Shoham, G., Garti, N., 2009. Soft matter dispersions with ordered inner structures, stabilized by ethoxylated phytosterols. Colloids Surf. B. Biointerfaces 74, 202-15. https://doi.org/10.1016/j.colsurfb.2009.07.020

Lopes da Silva, J.A., Coutinho, J.A.P., 2004. Dynamic rheological analysis of the gelation behaviour of waxy crude oils. Rheol. Acta 43, 433-441.

Luckham, P.F., Ukeje, M.A., 1999. Effect of particle size distribution on the rheology of dispersed systems. J. Colloid Interface Sci. 220, 347-356. https://doi.org/10.1006/jcis.1999.6515

Lutton, E.S., 1945. The Polymorphism of Tristearin and Some of its Homologs. JAOCS, J. Am. Oil Chem. Soc. 67, 524-527. https://doi.org/10.1021/ja01220a008

Ma, Q., Wang, W., Liu, Y., Yang, J., Shi, B., Gong, J., 2017. Wax adsorption at paraffin oilwater interface stabilized by Span80. Colloids Surfaces A Physicochem. Eng. Asp. 518, 7379. https://doi.org/10.1016/j.colsurfa.2017.01.023 
Macierzanka, A., Szelag, H., Szumała, P., Pawłowicz, R., Mackie, A.R., Ridout, M.J., 2009. Effect of crystalline emulsifier composition on structural transformations of water-in-oil emulsions: Emulsification and quiescent conditions. Colloids Surfaces A Physicochem. Eng. Asp. 334, 40-52. https://doi.org/10.1016/j.colsurfa.2008.09.053

Madivala, B., Vandebril, S., Fransaer, J., Vermant, J., 2009. Exploiting particle shape in solid stabilized emulsions. Soft Matter 5, 1717. https://doi.org/10.1039/b816680c

Maleky, F., Acevedo, N.C., Marangoni, A.G., 2012. Cooling rate and dilution affect the nanostructure and microstructure differently in model fats. Eur. J. Lipid Sci. Technol. 114, 748-759. https://doi.org/10.1002/ejlt.201100314

Maleky, F., Marangoni, A., 2011. Thermal and mechanical properties of Cocoa butter crystallized under an external laminar shear field. Cryst. Growth Des. 11, 2429-2437. https://doi.org/10.1021/cg200202u

Marangoni, A.G., Acevedo, N., Maleky, F., Co, E., Peyronel, F., Mazzanti, G., Quinn, B., Pink, D., 2012. Structure and functionality of edible fats. Soft Matter 8, 1275-1300. https://doi.org/10.1039/C1SM06234D

Marangoni, A.G., Rousseau, D., 1996. Is Plastic Fat Rheology Governed by the Fractal Nature of the Fat Crystal Network? 73, 991-994.

Marefati, A., Bertrand, M., Sjöö, M., Dejmek, P., Rayner, M., 2017. Storage and digestion stability of encapsulated curcumin in emulsions based on starch granule Pickering stabilization. Food Hydrocoll. 63, 309-320. https://doi.org/10.1016/j.foodhyd.2016.08.043

Matos, M., Laca, A., Rea, F., Iglesias, O., Rayner, M., Gutiérrez, G., 2018. O/W emulsions stabilized by OSA-modified starch granules versus non-ionic surfactant: Stability, rheological behaviour and resveratrol encapsulation. J. Food Eng. 222, 207-217. https://doi.org/10.1016/j.jfoodeng.2017.11.009

McClements, D.J., 2005. Food Emulsions: Principles, Practices, and Techniques, 2nd ed. CRC Press, Boca Raton, Florida.

McClements, D.J., Monahan, F.J., Kinsella, J.E., 1993. Effect of Emulsion Droplets on the Rheology of Whey-Protein Isolate Gels. J. Texture Stud. 24, 411-422. https://doi.org/DOI 10.1111/j.1745-4603.1993.tb00051.x

Melle, S., Lask, M., Fuller, G.G., 2005. Pickering emulsions with controllable stability. Langmuir 21, 2158-62. https://doi.org/10.1021/la047691n

Middendorf, D., Juadjur, A., Bindrich, U., Mischnick, P., 2015. AFM approach to study the function of PGPR's emulsifying properties in cocoa butter based suspensions. Food Struct. 4, 16-26. https://doi.org/10.1016/j.foostr.2014.11.003

Milak, S., Zimmer, A., 2015. Glycerol monooleate liquid crystalline phases used in drug delivery systems. Int. J. Pharm. 478, 569-587. https://doi.org/10.1016/j.ijpharm.2014.11.072

Miskandar, M.S., Man, Y.C., Yusoff, M.S.A., Rahman, R.A., 2005. Quality of margarine: Fats selection and processing parameters. Asia Pac. J. Clin. Nutr. 14, 387-395. 
Nadin, M., Rousseau, D., Ghosh, S., 2014. Fat crystal-stabilized water-in-oil emulsions as controlled release systems. LWT - Food Sci. Technol. 56, 248-255. https://doi.org/10.1016/j.lwt.2013.10.044

Narine, S., Marangoni, A., 1999. Fractal nature of fat crystal networks. Phys. Rev. E 59, 19081920. https://doi.org/10.1103/PhysRevE.59.1908

Niiya, I., Maruyama, T., Imamura, M., Okada, M., Matsumoto, T., 1973a. Effect of emulsifiers on the crystal growth of edible solid fats V - Effect of sugar fatty acid ester.pdf. J. Jpn. Soc. Food Sci. Technol. 20, 199-207.

Niiya, I., Maruyama, T., Imamura, M., Okada, M., Matsumoto, T., 1973b. Effect of Emulsifiers on the Crystal Growth of Edible Solid Fats IV: Effect of Propylene Glycol Ester of Fatty Acid and Unsaturated Fatty Acid Monoglycerides. J. Jpn. Soc. Food Sci. Technol. 20, 191198.

Niiya, I., Maruyama, T., Imamura, M., Okada, M., Matsumoto, T., Matusumoto, T., 1973c. Effect of Emulsifiers on the Crystal Growth of Edible Solid Fats III: Effect of Saturated Fatty Acid Monoglyceride. J. Jpn. Soc. Food Sci. Technol. 20, 182-190.

Norton, I.T., Spyropoulos, F., Cox, P.W., 2009. Effect of emulsifiers and fat crystals on shear induced droplet break-up, coalescence and phase inversion. Food Hydrocoll. 23, 15211526. https://doi.org/10.1016/j.foodhyd.2008.09.014

Omar, Z., Let, C.C., Seng, C.C., Rashid, N.A., 2005. Crystallisation and rheological properties of hydrogenated palm oil and palm oil blends in relation to crystal networking. Eur. J. Lipid Sci. Technol. 107, 634-640. https://doi.org/10.1002/ejlt.200501180

Pays, K., Giermanska-Kahn, J., Pouligny, B., Bibette, J., Leal-Calderon, F., 2002. Double emulsions : how does release occur? J. Control. Release 79, 193-205.

Perrechil, F.D.A., Santana, R.D.C., Fasolin, L.H., Silva, C.A.S. Da, Cunha, R.L. Da, 2010. Rheological and structural evaluations of commercial italian salad dressings. Ciência e Tecnol. Aliment. 30, 477-482. https://doi.org/10.1590/S0101-20612010000200027

Pickering, S.U., 1907. Emulsions. J. Chem. Soc. Trans. 91, 2001-2021.

Piska, I., Zárubová, M., Loužecký, T., Karami, H., Filip, V., 2006. Properties and crystallization of fat blends. J. Food Eng. 77, 433-438. https://doi.org/10.1016/j.jfoodeng.2005.07.010

Pitzalis, P., Monduzzi, M., Krog, N., Larsson, H., Ljusberg-wahren, H., Nylander, T., 2000. Characterization of the Liquid-Crystalline Phases in the Glycerol Monooleate / Diglycerol Monooleate / Water System. Langmuir 6358-6365.

Pradhan, M., Rousseau, D., 2012. A one-step process for oil-in-water-in-oil double emulsion formation using a single surfactant. J. Colloid Interface Sci. 386, 398-404. https://doi.org/10.1016/j.jcis.2012.07.055

Prichapan, N., McClements, D.J., Klinkesorn, U., 2017. Influence of rice bran stearin on stability, properties and encapsulation efficiency of polyglycerol polyricinoleate (PGPR)stabilized water-in-rice bran oil emulsions. Food Res. Int. 93, 26-32. https://doi.org/10.1016/j.foodres.2017.01.007 
Rafanan, R., Rousseau, D., 2019. Dispersed droplets as tunable fillers in water-in-oil emulsions stabilized with fat crystals. J. Food Eng. 244, 192-201.

https://doi.org/10.1016/j.foodres.2017.04.008

Rafanan, R., Rousseau, D., 2017. Dispersed droplets as active fillers in fat-crystal networkstabilized water-in-oil emulsions. Food Res. Int. 99, 355-362. https://doi.org/10.1016/j.foodres.2017.04.008

Ramamirtham, S., Shahin, A., Basavaraj, M.G., Deshpande, A.P., 2017. Phase Behavior and Micro-Structure of Fat-Oil Mixtures: Engineering the Shape of Fat Clusters. JAOCS, J. Am. Oil Chem. Soc. 94, 121-132. https://doi.org/10.1007/s11746-016-2926-2

Ramsden, W., 1903. Separation of solids in the surface layers of solutions and "suspensions" Observations on surface membranes, bubbles, emulsions, and mechanical coagulation. Proc. R. Soc. London 72, 156-164. https://doi.org/10.1098/rstl.1763.0053

Rayner, M., Marku, D., Eriksson, M., Sjöö, M., Dejmek, P., Wahlgren, M., 2014. Biomass-based particles for the formulation of Pickering type emulsions in food and topical applications. Colloids Surfaces A Physicochem. Eng. Asp. 458, 48-62.

https://doi.org/10.1016/j.colsurfa.2014.03.053

Ring, S., Stainsby, G., 1982. Filler reinforcement of gels. Prog. Food Nutr. Sci. 6, 323-329.

Rousseau, D., Ghosh, S., Park, H., 2009. Comparison of the dispersed phase coalescence mechanisms in different tablespreads. J. Food Sci. 74, E1-7. https://doi.org/10.1111/j.17503841.2008.00978.x

Sala, G., van Vliet, T., Cohen Stuart, M.A., Aken, G.A. van, van de Velde, F., 2009. Deformation and fracture of emulsion-filled gels: Effect of oil content and deformation speed. Food Hydrocoll. 23, 1381-1393. https://doi.org/10.1016/j.foodhyd.2008.11.016

Sarmento, B., Martins, S., Ferreira, D., Souto, E.B., 2007. Oral insulin delivery by means of solid lipid nanoparticles. Int. J. Nanomedicine 2, 743-749. https://doi.org/10.2147/IJN.S

Sato, K., Bayés-García, L., Calvet, T., Cuevas-Diarte, M.À., Ueno, S., 2013. External factors affecting polymorphic crystallization of lipids. Eur. J. Lipid Sci. Technol. 115, 1224-1238. https://doi.org/10.1002/ejlt.201300049

Servais, C., Jones, R., Roberts, I., 2002. The influence of particle size distribution on the processing of food. J. Food Eng. 51, 201-208.

Shih, W.-H., Shih, W.Y., Kim, S.-I., Liu, J., Aksay, I.A., 1990. Scaling behavior of the elastic properties of colloidal gels. Phys. Rev. A 42, 4772-4779.

https://doi.org/10.1103/PhysRevA.42.4772

Shiota, M., Iwasawa, A., Kotera, M., Konno, M., Isogai, T., Tanaka, L., 2011. Effect of fatty acid composition of monoglycerides and shear on the polymorph behavior in water-in-palm oil-based blend. JAOCS, J. Am. Oil Chem. Soc. 88, 1103-1111. https://doi.org/10.1007/s11746-011-1774-3

Simovic, D.S., Pajin, B., Seres, Z., Filipovic, N., 2009. Effect of low-trans margarine on physicochemical and sensory properties of puff pastry. Int. J. Food Sci. Technol. 44, 1235- 
1244. https://doi.org/10.1111/j.1365-2621.2009.01953.x

Simovic, S., Prestidge, C.A., 2007. Nanoparticle layers controlling drug release from emulsions.

Eur. J. Pharm. Biopharm. 67, 39-47. https://doi.org/10.1016/j.ejpb.2007.01.011

Smith, K.W., Bhaggan, K., Talbot, G., Van Malssen, K.F., 2011. Crystallization of fats: Influence of minor components and additives. J. Am. Oil Chem. Soc. 88, 1085-1101. https://doi.org/10.1007/s11746-011-1819-7

Spyropoulos, F., Heuer, E.A.K., Mills, T.B., 2011. Protein-stabilized emulsions and rheological aspects of structure and mouthfeel, in: Norton, I.T., Spyropoulos, F., Cox, P. (Eds.), Practical Food Rheology: An Interpretive Approach. West-Sussex, UK, pp. 193-214.

Subramanian, R., Muthukumarappan, K., Gunasekaran, S., 2006. Linear Viscoelastic Properties of Regular- and Reduced-Fat Pasteurized Process Cheese During Heating and Cooling. Int. J. Food Prop. 9, 377-393. https://doi.org/10.1080/10942910600596571

Tadros, T.F., 2013. Emulsion Formation , Stability , and Rheology.

Tavernier, I., Wijaya, W., Van der Meeren, P., Dewettinck, K., Patel, A.R., 2016. FOod-grade particles for emulsion stabilization. Trends Food Sci. Technol. 50, 159-174. https://doi.org/10.1016/j.colsurfa.2016.05.084

Taylor, M.S., 2011. Stabilisation of water-in-oil emulsions to improve the emollient properties of Lipstick. Thesis 144.

Thareja, P., 2013. Rheology and microstructure of pastes with crystal network. Rheol. Acta 52, 515-527. https://doi.org/10.1007/s00397-013-0716-4

Thareja, P., Golematis, A., Street, C.B., Wagner, N.J., Vethamuthu, M.S., Hermanson, K.D., Ananthapadmanabhan, K.P., 2012. Influence of Surfactants on the Rheology and Stability of Crystallizing Fatty Acid Pastes. J. Am. Oil Chem. Soc. 90, 273-283. https://doi.org/10.1007/s11746-012-2161-4

Tran, T., Green, N.L., Ghosh, S., Rousseau, D., 2017. Encapsulation of water-in-oil emulsion droplets within crystal spheroids. Colloids Surfaces A Physicochem. Eng. Asp. 524, 1-7. https://doi.org/10.1016/j.colsurfa.2016.12.054

Van Aken, G.A., Oliver, L., Scholten, E., 2015. Rheological effect of particle clustering in gelled dispersions. Food Hydrocoll. 48, 102-109. https://doi.org/10.1016/j.foodhyd.2015.02.001

van Kuijk-Meuwissen, M.E., Mougin, L., Junginger, H.E., Bouwstra, J.A., 1998. Application of vesicles to rat skin in vivo: a confocal laser scanning microscopy study. J Control Release 56, 189-196. https://doi.org/S0168-3659(98)00087-X [pii]

van Vliet, T., 1988. Rheological properties of filled gels. Influence of filler matrix interaction. Colloid Polym. Sci. 266, 518-524. https://doi.org/10.1007/BF01420762

Vignati, E., Piazza, R., Visintin, R.F.G., Lapasin, R., D’Antona, P., Lockhart, T.P., 2005. Wax crystallization and aggregation in a model crude oil. J. Phys. Condens. Matter 17, S3651S3660. https://doi.org/10.1088/0953-8984/17/45/061

Visintin, R.F.G., Lapasin, R., Vignati, E., D’Antona, P., Lockhart, T.P., 2005. Rheological 
Behavior and Structural Interpretation of Waxy Crude Oil Gels. Langmuir 21, 6240-6249. https://doi.org/10.1021/la050705k

Visintin, R.F.G., Lockhart, T.P., Lapasin, R., D’Antona, P., 2008. Structure of waxy crude oil emulsion gels. J. Nonnewton. Fluid Mech. 149, 34-39. https://doi.org/10.1016/j.jnnfm.2007.07.008

Wassell, P., Okamura, A., Young, N.W.G., Bonwick, G., Smith, C., Sato, K., Ueno, S., 2012. Synchrotron radiation macrobeam and microbeam $\mathrm{X}$-ray diffraction studies of interfacial crystallization of fats in water-in-oil emulsions. Langmuir 28, 5539-5547. https://doi.org/10.1021/la204501t

Wickramarachchi, K.S., Sissons, M.J., Cauvain, S.P., 2015. Puff pastry and trends in fat reduction: An update. Int. J. Food Sci. Technol. 50, 1065-1075. https://doi.org/10.1111/ijfs.12754

Winuprasith, T., Khomein, P., Mitbumrung, W., Suphantharika, M., Nitithamyong, A., McClements, D.J., 2018. Encapsulation of vitamin D3in pickering emulsions stabilized by nanofibrillated mangosteen cellulose: Impact on in vitro digestion and bioaccessibility. Food Hydrocoll. 83, 153-164. https://doi.org/10.1016/j.foodhyd.2018.04.047 